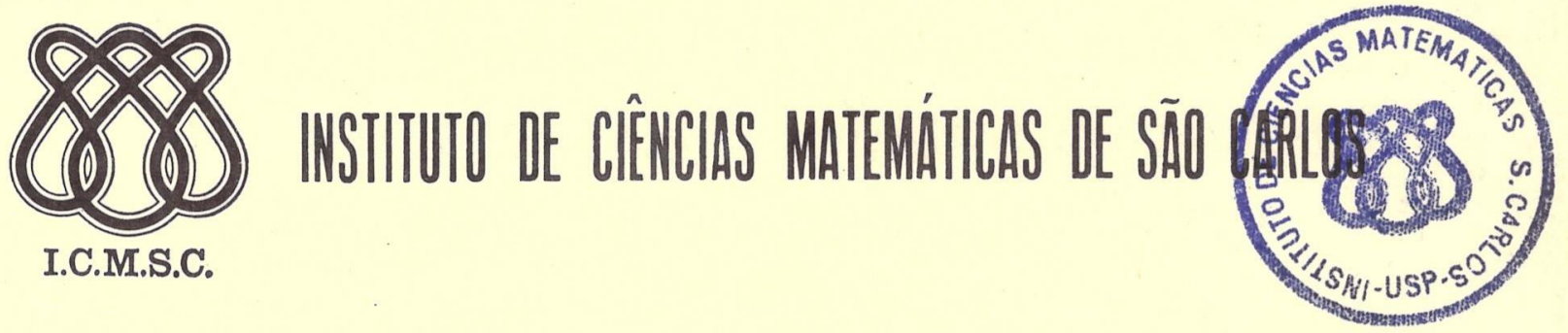

\title{
A ÁLGEBRA DE CLIFFORD CANÔNICA
}




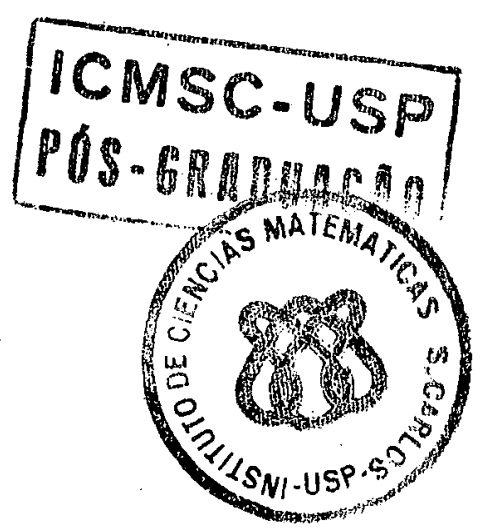

A ÁLGEBRA DE CLIFFORD CANÔNICA

\section{Celi Vasques Crepaldi}

ORIENTADOR: Prof. Dr. Cândido Lima da Silva Dias

Tese apresentada ao Instituto de Ciēncias Matemáticas de São Carlos, da Universidade de São Paulo, para obtenção do título de Doutor em Ciências (Matemática). 
Aos meus pais

ao Moacix e

às minhas filhas 


\section{AGRADEGO SINCERAMENTE}

Ao Prof. Dr. Cândido Lima da Silva Dias, pela orientação segura e principalmente pelo incentivo e amizade.

Ao Prof. Dr. Antonio Paques, pelas suas valiosas sugestões e co 1 aborações.

Ao Prof. Dr. Antonio Conde sempre pronto a prestar-me o seu valioso auxílio.

Aos colegas e amigos do IQAr. - UNESP, em especial, ao Prof. Ro meu Magnani, pela amizade; apoio e colaboração.

Aos colegas e amigos do I.C.M.S.C., pelo apoio e estímulo.

Ao José Carlos pela paciência, dedicação e pelo ótimo trabalho de datilografia. 


\section{ABSTRACT}

Our intention was to construct and study one Clifford algebra $\mathrm{C}_{\mathrm{M}}$, which has a fundamental importance to the determination of other Clifford a1 gebras. Moreover, it allows the stablishment of the relation between the Clifford algebra of vector space $E$, of a finite dimension, and the exterior algebra of that space: $\Lambda \mathrm{E}$.

The algebra $C_{M}$ is a Clifford algebra of the space $M=E \oplus E^{*}$, which the quadratic form $Q\left(\left(x, x^{\prime}\right)\right)=\left\langle x, x^{\prime}\right\rangle$.

When we have studied the properties of this algebra, we have come to the conclusion that the quadratic space $M$ (and the space E) over the field $\mathrm{K}$, has an inner product.

The orthogonal group $G$ de $\mathrm{C}_{M}$ and the Clifford group de $G$ are very important and have been described and analysed in Chapter II.

In Chapter III, we have construct one algebra on the vector space $E$ with a quadratic form $Q_{1}$, induced from $Q$, and by analogy, we have construct one algebra on the vector space $E^{*}$, we have also stablished the relations among the algebras $C_{M}, C_{E}$ e $C_{E} *$.

On Chapter IV, we have shown that the Clifford algebra of $\mathrm{E}$ can be obtained as a deformation of the exterior algebra $\Lambda E$, based on the works of $\mathrm{J}$. Helmstetter and using the interior products. Finally, we have shown an analogy between the reasoning of Helmstetter and the results we have attained. 
I N D I C E

INTRODUÇÃO

CAPÍtULO I

ĀLGEBRA DE CLIFFORD

CAPITULO II

GRUPO DE CLIFFORD

CAPITULO III

RELAÇÕES ENTRE AS ĀLGEBRAS DE CLIFFORD E ĀLGEBRA EXTERIOR

CAPITULO IV

AS ÁLGEBRAS DE CLIFFORD COMO DEFORMAÇÕES DAS ÁLGEBRAS EXTERIORES 


\section{INTRODUGÃO}

A idéia inicial e a motivação para este trabalho surgiram de um artigo de M. Schemberg [29], no qual o autor introduziu uma älgebra de Clif ford de um espaço pseudo-euclidiano de dimensão $2 \mathrm{n}$.

A definição da álgebra $G_{n}$ é intrínseca e obtivemos uma defini. ção equivalente e canónica.

A partir do K-espaço vetorial $\mathrm{E}$ de dimensão $\mathrm{n}$, define-se o espa ço $M=E \times E^{*}$, onde $E^{*} \bar{e} \circ$ dual de $E$ e sobre $M$ considera-se a forma quadrática canônica $Q\left[\left(x, x^{\prime}\right)\right]=\left\langle x, x^{\prime}\right\rangle$; no Capítulo I, construímos a álgebra de Clifford de $M$ em relação à forma quadrática canónica e a representamos por $C_{M}$, cuja multipli cação é dada por

$$
x_{i} y_{j}^{\prime}+y_{j}^{\prime} x_{i}=\delta_{i j} \cdot 1
$$

onde $\left(x_{i}\right)_{1 \leq i \leq n} \bar{e}$ uma base de $E$ e $\left(y_{j}^{\prime}\right)_{1 \leq j \leq n} \bar{e}$ a base dual de $E^{*}$.

Estudam-se também no Capítulo I, as propriedades do espaço quadrático $(M, Q)$ : é um espaço hiperbólico e se $K=\mathbb{R}$, é um espaço pseudo-euclidiano com Indice de inērcia igual a $(n, n)$.

Como $M$ é de dimensão par, verifica-se que $C_{M} \bar{e}$ uma álgebra central simples isomorfa à àlgebra das matrizes de ordem $2^{\mathrm{n}}$.

A construção e as propriedades gerais da ālgebra de Clifford são estudadas no Capítulo I; define-se tambëm a älgebra de Weyl e as suas propriedades, mostrando suas relações com a ālgebra de clifford.

No Capítulo II, determina-se o grupo ortogonal de $Q$, representa do por G, isto é, o grupo dos automorfismos lineares de $M$ que conservam a forma quadrätica e o grupo de Clifford $\Gamma$ associado a $G$; obtivemos a descrição dos auto morfismos lineares de $M$ que pertencem ao grupo $G$ e como a dimensão de $M$ é par, para cada $F \in G$, existe $s \in \Gamma$ tal que $\chi(s) \equiv F$, onde $\chi$ é a representação linear de $\Gamma$; por este procedimento, determina-se o grupo de Clifford de $G$ e estudam-se as propriedades. 
Neste Capítulo, obtém-se um resultado importante: como E e E* são subespaços totalmente singulares maximais de $M$, eles são permutados entre si por operações $F$ do grupo ortogonal; então a restrição de F a E fornece um isomor fismo linear $\mathrm{u}: \mathrm{E} \rightarrow \mathrm{E}^{*}$, e através de $\mathrm{u}$, obtēm-se uma forma bilinear $\mathrm{B}_{1}: \mathrm{E} \times \mathrm{E} \rightarrow \mathrm{K}$ não-degenerada (e simétrica se $u={ }^{t} u$ ), que origina uma ālgebra de Clifford sobre o espaço $E$, representada $C_{E}$ (Se $B_{1}$ não é simétrica, pode-se obter uma forma bilinear simétrica $\mathrm{B}^{\prime}: \mathrm{E} \times \mathrm{E} \rightarrow \mathrm{K}$, a partir de $\mathrm{B}_{1}$ ).

No Capítulo III, evidenciamos as relações entre a älgebra de Clifford de um espaço vetorial $\mathrm{E}$ e a álgebra exterior deste espaço, utilizando-se a construção de Chevalley em [8]; obtém-se a igualdade

$$
x \cdot y=x \wedge y+B(x, y) \quad x, y \in E
$$

onde $B$ é a forma bilinear canônica associada a $Q$ e demonstra-se que $C_{E}$ e $\Lambda E$ são isomorfos como espaços vetoriais.

A álgebra $C_{M}$ é de uma importância fundamental pois pode-se de monstrar que: uma ālgebra de Clifford de um espaço vetorial de dimensão finita E, em relação à uma forma quadrática $Q_{1}$ pode ser obtida da álgebra $C_{M}$ e analogamente para o espaço vetorial $\mathrm{E}^{*}$. No Capítulo III, verifica-se que os espaços quadrá ticos $(M, Q),\left(E, Q_{1}\right)$ e $\left(E^{*}, Q_{2}\right)$ são isométricos e são estabelecidas as relações en tre $C_{M}, C_{E}$ e $C_{E}^{*}$.

Finalmente, demonstra-se o isomorfismo de álgebra entre $C_{M}$ e a álgebra dos endomorfismos de $\Lambda$ E, utilizando-se os produtos interiores.

No Capítulo IV, generaliza-se os resultados de Chevalley, baseando-se em trabalhos mais recentes do autor J. Helmstetter [17], [18]. O autor se propõe estudar os isomorfismos $\mathrm{J}_{\mathrm{B}}: \mathrm{C}_{\mathrm{E}} \rightarrow \Lambda E$ como espaços vetoriais, dependendo de uma forma bilinear $B$ sobre $E$ tal que $B(a, a)=Q(a)$ para todo a $E E$, que não é necessariamente a forma bilinear canonicamente associada a Q.

Considerando-se que a álgebra de Clifford $C_{E}$ e a álgebra exterior $\Lambda E$ tem o mesmo espaço vetorial, constrói-se sobre o espaço vetorial $\Lambda E$ um 
produto (que depende de B) que a transforma numa àlgebra $\Lambda(E, B)$, isomorfa à àlge bra de Clifford $C_{E}$; o produto de Clifford aparece então como uma "deformação" do produto exterior.

Para estudar esta ālgebra $\Lambda(E, B)$, utiliza-se os produtos interiores que originam uma anti-derivação de $\Lambda E$ e dependem de formas lineares sobre E.

Estes procedimentos podem ser usados com poucas mudanças na álgebra simplética.

Mostra-se que esta abordagem da älgebra de Clifford apresenta estreita analogia com os objetivos deste trabalho e com os resultados obtidos. 


\section{CAPITTULO I}

\section{ÁLGEBRA DE CLIFFORD}

Neste capítulo, daremos a definição e construção das ālgebras de Clifford, e também suas propriedades fundamentais.

Também serão introduzidos conceitos essenciais ao desenvolvimento do trabalho.

A seguir, determinaremos a álgebra de clifford de um espaço particular $M=E \oplus E^{*}$, onde $E \bar{e}$ um espaço vetorial qualquer dim $E=n$ sobre $K$ e $E^{*}$ o seu espaço dual, em relação à forma quadrática canônica $Q: Q\left[\left(x, x^{\prime}\right)\right]=\left\langle x, x^{\prime}\right\rangle$, onde $\left\langle\mathrm{x}, \mathrm{x}^{\prime}\right\rangle \overline{\mathrm{e}}$ a forma bilinear fundamental, e analisaremos as suas pronriedares.

Seja M um espaço vetorial sobre um corpo $\mathrm{K}$.

Definição 1.1. Uma forma quadrätica em M é uma aplicação Q de $M$ em $K$ que tem as seguintes propriedades:
(a) $Q(a x)=a^{2} \cdot Q(x)$
$a \in K, x \in M$
(b) A aplicação $(x, y) \rightarrow Q(x+y)-Q(x)-Q(y)$ é uma forma bilinear $B: M \times M \rightarrow K$.

Dizemos que B é a forma bilinear associada a Q. E claro da defi nição que $B(x, y)=B(y, x)$ para todo $x, y \in M$. $B$ é portanto simétrica.
Além disso, temos: $Q(2 x)=4 Q(x)$ o que implica por (b) $B(x, x)=2 Q(x)$.

A forma bilinear simétrica associada com a aplicação quadrática Q è única. 
Definição 1.2. Um espaço quadrātico è um espaço vetorial de dimensão finita sobre $K$, munido de uma forma quadrática $Q$ e a forma bilinear simétrica $B$ associada com Q.

A restrição da aplicação $Q$ a um subespaço $N$ de $M$ é uma forma qua drática em $N$ cuja forma bilinear associada é a restrição de $B$ a $N \times N$.

Definição 1.3. Un elemento $x \in M$ é chamado singular se $Q(x)=0$. Se a restrição da forma quadrática a $\mathrm{N}$ é iqual a zero, dizemos que $\mathrm{N}$ é totalmen te singular.

Seja K um corpo comutativo de característica diferente de 2 e se ja M um K-espaço vetorial.

Define-se:

$$
M^{\prime}=\{x \in M t \cdot q \cdot B(x, y)=0 \forall y \in M\}
$$

Definição 1.4. O posto da forma quadrática $Q$ é o número $\mathrm{m}^{-\mathrm{m}}$ ' tal que $\mathrm{m}=$ dim $M e^{\prime} m^{\prime}=\operatorname{dim} M^{\prime}$.

Definição 1.5. Um espaço vetorial M diferente de zero, é isotrópico (ou singular) se ele contém um vetor singular.

Proposição 1.6. [8] Seja $B_{0}$ uma forma bilinear em $M \times M$. Então $x \rightarrow B_{0}(x, x)$ é uma forma quadrática em $Q$ e toda forma quadrática pode ser representada desta maneira.

Se K não é característica 2, podemos tomar na Proposição 1.6, $\mathrm{B}_{\mathrm{O}}=\frac{1}{2} \mathrm{~B}$.

Seja E um K-espaço vetorial de dimensão $n$ e seja $\left\{x_{1}, \ldots, x_{n}\right\}$ uma base de $E$, seja $E^{*}$ o espaço dual de $E$ e consideremos a base dual da base $\left\{x_{i}\right\}$, is to é,

$$
\left\{x_{1}^{\prime}, \ldots, x_{n}^{\prime}\right\} \bar{e} \text { a base de } E^{*} \text { tal que } x_{i}^{\prime}\left(x_{j}\right)=\delta_{i j} \text { ou }\left\langle x_{i}, x_{j}^{\prime}\right\rangle=\delta_{i j}
$$


Define-se o conjunto

$$
M=E \times E^{*}=\left\{\left(x, x^{\prime}\right) / x \in E \text { e } x^{\prime} \in E^{*}\right\}
$$

Introduz-se em M uma estrutura de espaço vetorial, através das operações:

$$
\begin{aligned}
& \left(x, x^{\prime}\right)+\left(y, y^{\prime}\right)=\left(x+y, x^{\prime}+y^{\prime}\right) \\
& \lambda\left(x, x^{\prime}\right)=\left(\lambda x, \lambda x^{\prime}\right) \\
& \forall\left(x, x^{\prime}\right),\left(y, y^{\prime}\right) \in M, \forall \lambda \in K .
\end{aligned}
$$

0 espaço vetorial $M=E \times E^{*}$ é chamado o produto cartesiano de $E$ por $E^{*}$.

Proposição 1.7. Se $E=\left\{x_{1}, \ldots, x_{n}\right\}$ e $F=\left\{x_{1}^{\prime}, \ldots, x_{n}^{\prime}\right\}$ são bases de $E$ e $E^{\star}$, respec tivamente, então

$E+F=\left\{\left(x_{1}, 0\right), \ldots,\left(x_{n}, 0\right),\left(0, x_{1}^{\prime}\right), \ldots,\left(0, x_{n}^{\prime}\right)\right\} \bar{e}$ uma base de $\mathrm{E} \times \mathrm{E}^{*}$.

Demonstração: De fato,

$$
\alpha_{1}\left(x_{1}, 0\right)+\ldots+\alpha_{n}\left(x_{n}, 0\right)+\beta_{1}\left(0, x_{1}^{\prime}\right)+\ldots+\beta_{n}\left(0, x_{n}^{\prime}\right)=0
$$

significa

$$
\begin{aligned}
& \alpha_{1} x_{1}+\ldots+\alpha_{n} x_{n}+\beta_{1} 0+\ldots+\beta_{n} 0=0 \\
& \alpha_{1} \cdot 0+\ldots+\alpha_{n} \cdot 0+\beta_{1} x_{1}^{\prime}+\ldots+\beta_{n} x_{n}^{\prime}=0
\end{aligned}
$$

Como os $x_{i}$ e $x_{j}^{\prime}$ são linearmente independentes isto implica

$$
\alpha_{i}=0 \text { e } \beta_{j}=0 \text {. }
$$


Portanto, $E+F \bar{e}$ um sistema l.i.

Alëm disso, se $z \in E \times E^{*}$, então $z=\left(x, x^{\prime}\right), x \in E$ e $x^{\prime} \in E^{*}$ assim,

$$
\begin{aligned}
z & =\left(x, x^{\prime}\right)=\left(\sum_{i=1}^{n} \alpha_{i} x_{i}, \sum_{j=1}^{n} \beta_{j} x_{j}^{\prime}\right)= \\
& =\sum_{i=1}^{n} \alpha_{i}\left(x_{i}, 0\right)+\sum_{j=1}^{n} \beta_{j}\left(0, x_{j}^{\prime}\right)
\end{aligned}
$$

portanto, todo $z \in \mathrm{E} \times \mathrm{E}^{*}$ pode ser escrito como combinação linear dos elementos de $E+F$.

Conclui-se que:

$$
\operatorname{dim}\left(E \times E^{*}\right)=\operatorname{dim} E+\operatorname{dim} E^{*}=2 n
$$

Se $M^{\prime}=E \oplus E^{*}$, existe um isomorfismo canônico $T: E \oplus E^{*} \rightarrow E \times E^{*}$

tal que

$$
\begin{aligned}
& T\left(x+x^{\prime}\right)=\left(x, x^{\prime}\right) \quad x \in E, \quad x^{\prime} \in E^{*} \\
& E \times E^{*} \cong E \oplus E^{*}
\end{aligned}
$$

is to é,

Pode-se identificar (a menos de isomorfismos) os subespaços E' e $E^{*}$, com $E$ e $E^{*}$, respectivamente, sendo:

$$
\begin{aligned}
& E^{\prime}=\left\{\left(x, 0^{\prime}\right) \in E \times E^{*}\right\}=\left\{\left(x, 0^{\prime}\right) / x \in E \text { e } 0^{\prime} \in E^{*}\right\} \\
& E^{\prime^{*}}=\left\{\left(0, x^{\prime}\right) \in E \times E^{*}\right\}=\left\{\left(0, x^{\prime}\right) / 0 \in E \text { e } x^{\prime} \in E^{*}\right\} \\
& E^{\prime} \cong E \text { e } E^{*} \cong E^{*}
\end{aligned}
$$


Portanto, deste modo, $\mathrm{E}$ e $\mathrm{E}^{*}$ podem ser considerados subespaços vetoriais de $\mathrm{M}=\mathrm{E} \oplus \mathrm{E}^{*}=\mathrm{E} \times \mathrm{E}^{*}$, isto $\overline{\mathrm{e}}$, o produto cartesiano $\mathrm{E} \times \mathrm{E}^{*}$ se decom põe como soma direta de subespaços isomorfos canonicamente a $E$ e $E^{*}$.

Sobre M toma-se a forma quadrática canônica

$$
Q: M \rightarrow K / Q\left(x, x^{\prime}\right)=\left[x^{\prime}(x)\right]
$$

Q é de fato uma forma quadrātica, pois:

$$
\text { (a) } \mathrm{Q}\left[\lambda\left(\mathrm{x}, \mathrm{x}^{\prime}\right)\right]=\left\langle\lambda \mathrm{x}, \lambda \mathrm{x}^{\prime}\right\rangle=\lambda^{2} \cdot\left\langle\mathrm{x}, \mathrm{x}^{\prime}\right\rangle=\lambda^{2} \cdot \mathrm{Q}\left[\left(\mathrm{x}, \mathrm{x}^{\prime}\right)\right]
$$

isto é,

$$
\begin{aligned}
& Q[\lambda z]=\lambda^{2} \cdot Q(z) \quad \lambda \in K, z \in M \\
& \text { (b) Se } z=\left(x, x^{\prime}\right) \text { e } w=\left(y, y^{\prime}\right) \in M \text {, } \\
& B(z, \omega)=Q(z+\omega)-Q(z)-Q(\omega) \\
& =x^{\prime}(y)+y^{\prime}(x)
\end{aligned}
$$

é uma forma bilinear $\mathrm{B}$ em $\mathrm{M} \times \mathrm{M}$.

Observemos que $B$ é não-degenerada.

Temos também $B_{0}\left(\left(x, x^{\prime}\right),\left(y, y^{\prime}\right)\right)=\frac{1}{2}\left[x^{\prime}(y)+y^{\prime}(x)\right]$.

Então M com a forma quadrática $Q$ e a forma bilinear B é um espaço quadrātico.

Podemos associar uma matriz simétrica com uma base $E+F$ de $M$, tomando a matriz $2 n \times 2 n$, cujos elementos são

$$
B_{o}\left(\left(x_{i}, x_{j}^{\prime}\right)\left(x_{k}, x_{\ell}^{\prime}\right)\right) \cdot 1 \leq i, j, k, l \leq n .
$$

Seja $\quad\left\{x_{1}, \ldots, x_{n}, x_{1}^{\prime}, \ldots, x_{n}^{\prime}\right\}$ base de $M=E \oplus E^{*}$. Então temos 


$$
\begin{aligned}
& B\left(\left(x_{i}, 0^{\prime}\right),\left(x_{j}, 0^{\prime}\right)\right)=0 \\
& B\left(\left(0, x_{i}^{\prime}\right),\left(0, x_{j}^{\prime}\right)\right)=0 \quad \forall i, j, 1 \leq i, j, \leq n \\
& B\left(\left(x_{i}, 0^{\prime}\right),\left(0, x_{j}^{\prime}\right)\right)=x_{j}^{!}\left(x_{i}\right)=\delta_{i j}=\begin{array}{r}
1 \\
1 \text { se } i=j \\
0 \text { se } i \neq j
\end{array} \\
& B\left(\left(0, x_{i}^{\prime}\right),\left(x_{j}, 0^{\prime}\right)\right)=x_{i}^{!}\left(x_{j}\right)=\delta_{i j}=\begin{array}{r}
\left\{\begin{array}{l}
1 \\
\text { se } i=j \\
0 \text { se } i \neq j
\end{array}\right.
\end{array}
\end{aligned}
$$

Portanto, a matriz $N$, que é a matriz do espaço quadrático $M$ na base em relação à forma bilinear B será:

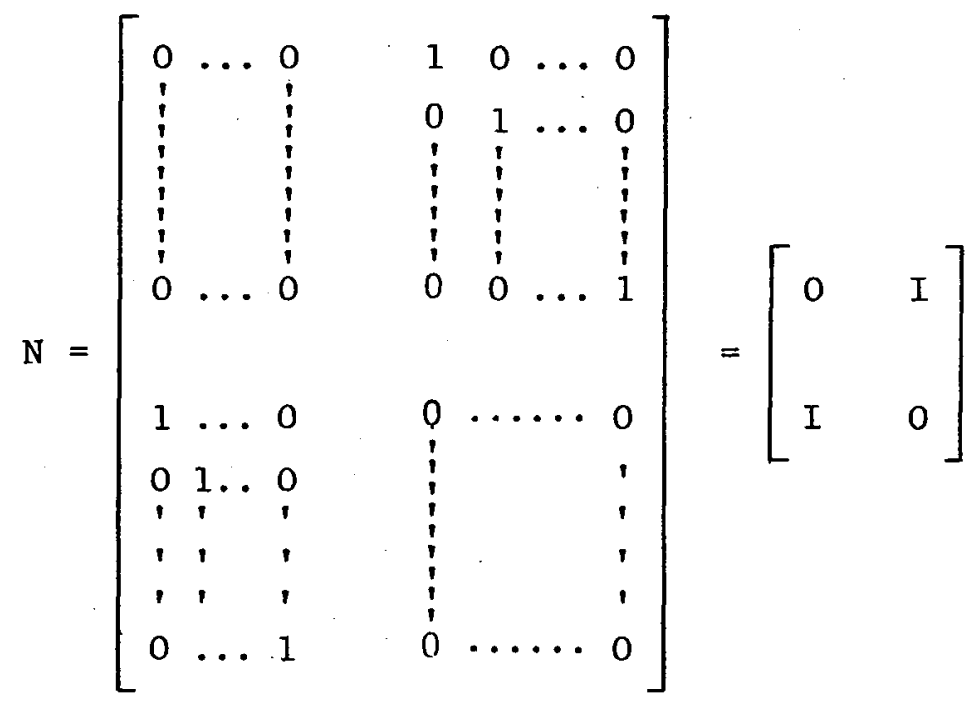

Usa-se a notação

$$
\mathrm{M} \cong \mathrm{N}
$$

0 determinante da matriz $\mathrm{N}$ é chamado o discriminante dos vetores $\left\{\dot{x}_{1}, \ldots, x_{n}, x_{1}^{\prime}, \ldots, \dot{x}_{n}^{\prime}\right\}$ e temos $\operatorname{det} N=-1$. (se $n$ é impar).

Uma condição necessāria e suficiente para que B seja não-degenerada é que seu discriminante seja diferente de zero.

observemos que os subespaços $\mathrm{E} \subset \mathrm{Me} \mathrm{E}^{*} \subset \mathrm{M}$ são totalmente singu lares, pois: 


$$
\begin{aligned}
& Q(E)=0 \quad \text { e } Q\left(E^{*}\right)=0 \\
& \text { e vale } Q(E)=0 \Longleftrightarrow B_{0}(E, E)=0 \\
& \text { por causa da identidade }
\end{aligned}
$$

$$
\mathrm{B}_{\mathrm{O}}(z, \omega)=\frac{1}{2}[Q(z+\omega)-Q(z)-Q(\omega)] \forall z, \omega \in M
$$

Seja U um subespaço do espaço quadrático M. O complemento ortogo nal $U^{\prime}$ (espaço conjugado) de U em M é o subespaço.

$$
U^{\prime}=\{z \in M \mid B(z, U)=0\}
$$

Definição 1.8. Definimos o radical de M como o subespaço

$$
\operatorname{rad} M=\{z \in M \mid B(z, M)=0\}=M !
$$

M é um espaço regular quadrático se $\operatorname{rad} M=0$.

0 espaço quadrātico $M$ é regular se e somente se det. $\mathrm{N} \neq 0$.

Um importante exemplo de um espaço regular isotrópico é o plano

hiperbōlico.

Definição 1.9. Chama-se plane hiperbólico um espaço vetorial de dimensão 2, mu nido de uma forma quadrática, que é regular e possui uma base de vetores singuláres.

As seguintes afirmações são equivalentes para um espaço quadrā-

tico binário E:
(a) E é um plano hiperbólico
(b) E é isotrópico e regular
(c) $\mathrm{dE}=-1$.

Um plano hiperbólico E tem a matriz $E \cong\left[\begin{array}{ll}0 & 1 \\ 1 & 0\end{array}\right]$ em uma de suas bases. 
Definição 1.9. Seja um vetor y $\in M$ tal que $Q(y) \neq 0$

Define-se uma aplicação $\tau_{y}: M \rightarrow M$, pela fórmula

$$
\tau_{y} x=x-\frac{B(x, y)}{Q(y)} \cdot y
$$

$\tau_{y}$ é um endomorfismo de $M$, que conserva a forma quadrática; é uma involução e é chamada a simetria em relação a y.

$\tau_{y}$ deixa os pontos do hiperplano $\mathrm{H}$, conjugado de $\mathrm{Ky}$, invariantes. Toda simetria num plano hiperbólico troca as duas retas isotrópi cas.

Temos a seguinte:

Proposição 1.10. [26] M é um espaço regular quadrático, E é um subespaço com $Q(E)=0$ e $\left\{x_{1}, \ldots, x_{n}\right\}$ é uma base para $E$. Então existe um subespaço $H_{1} \perp \ldots \perp H_{n}$ de $M$ no qual cada $H_{i}$ é um plano hiperbölico $\operatorname{com} x_{i} \in H_{i}$.

Temos então $M=E \oplus E^{*}, E \subset M, Q(E)=0$ e $\left\{x_{1}, \ldots, x_{n}\right\}$ base de $E$. Se mantermos a decomposição de planos hiperbólicos em $M$, temos

$$
\mathrm{M}=\mathrm{H}_{1} \perp \cdots \perp \mathrm{H}_{\mathrm{n}} \perp \mathrm{M}_{\mathrm{O}}
$$

como $0 \leq 2 n \leq \operatorname{dim} M$ em cada $H_{i}$ é um plano hiperbólico e $M_{0}$ é ou 0 ou não singular.

Pelo Teorema de Witt vemos que $\mathrm{n}$ não depende do modo como a de composição é feita, e então $M_{0}$ é único a menos de isometria.

Aplica-se agora a Proposição 1.10 e concluímos que n é realmente

- Indice de M. Em particular isto prova que $n=$ ind M satisfaz a desigualdade.

$$
0 \leq 2 \text { ind } M \leq \operatorname{dim} M
$$


Definição 1.11. Neste caso, chama-se $M$ um espaço hiperbólico se $0<2 n=$ dim $M$. Então M é hiperbólico se e somente se tem uma decomposição.

$$
\mathrm{M}=\mathrm{H}_{1} \perp \cdots \perp \mathrm{H}_{\mathbf{n}}
$$

no qual $n \geq 1$ com todos $H_{i}$ planos hiperbólicos.

Em particular, o espaço vetorial çonsiderado $M=E \oplus E^{*}$ é um es paço hiperbólico.

0 Índice de um espaço regular quadrädico $M$ é um invariante que pode ser definido assim:

Definição 1.12. Chama-se índice de $Q$ a dimensão comum de todos os subespaços ma ximais regularmente singulares de $M$.

Seja M um K-espaço vetorial, munido de uma forma quadrática e uma forma bilinear associada.

\section{Debinição:1.13. (da álgebra de Clifford)}

Seja T(M) a álgebra tensorial do espaço vetorial M e I o ideal gerado em $T$ pelos elementos $\mathrm{x} \otimes \mathrm{x}-\mathrm{Q}(\mathrm{x}) .1$ para todo $\mathrm{x} \in \mathrm{M}$. Então a ālgebra $C(M)=\frac{T(M)}{I} \bar{e}$ chamada ā1gebra de clifford da forma quadrática $Q$.

A ālgebra de Clifford é uma ālgebra graduada.

De fato, seja $T^{h}(M)$ o espaço dos elementos homogêneos de grau $h$ de $T(M)$. Representamos por $T_{+}(M)=\underset{h \text { par }}{\oplus} T^{h}(M)$ e $T_{-}(M)=\underset{h \text { impar }}{\oplus} T^{h}(M)$.

Então

$$
T(M)=T_{+}(M) \quad \oplus \quad T_{-}(M)
$$

0 ideal I é gerado por elementos pertencentes a $T_{+}(M)$. Desde que $T(M)$ tem uma base composta de elementos homogéneos, todo elemento de $I$ pode ser escrito como a soma de elementos de $I \cap T_{+}(M)$ e $I \cap T_{-}(M)$. 
Seja

$$
C_{+}(M)=\frac{T_{+}(M)}{I \cap T_{+}(M)} \text { e } \quad C_{-}(M)=\frac{T_{-}(M)}{I \cap T_{+}(M)}
$$

\section{Então}

$$
C(M)=C_{+}(M) \oplus C_{-}(M)
$$

e diz-se também que C(M) tem uma estrutura de uma álgebra $Z_{2}$ - graduada, representando-se

$$
C_{+}(M)=C_{0}(M) \quad \text { e } \quad C_{-}(M)=C_{1}(M)
$$

Lembremos que uma $\mathrm{K}-\bar{a}$ lgebra $\mathrm{C}$ é $\underline{Z_{2} \text {-graduada }}$ se ela é de dimensão finita dada na forma $C=C_{0} \oplus C_{1}$, tal que $K=K .1 \subset C_{0}$ e que $C_{i} \cdot C_{j} \subset C_{i+j}$,

(Em particular, $c_{0} \bar{e}$ uma sub-ālgebra).

Os elementos de $C_{+}(M)$ são chamados pares e os elementos de $C_{-}(M)$ são impares.

Representaremos, a partir daqui, a ālgebra de Clifford do espaço vetorial M, por $C$, enquanto não existir dúvidas sobre o espaço vetorial consi derado.

Definição 1.14. Chama-se involução principal o automorfismo linear $\varepsilon: \mathrm{C} \rightarrow \mathrm{C}$ tal que

$$
\begin{aligned}
& \varepsilon(u)=u \text { se } \quad u \in C_{+} \\
& \varepsilon(u)=-u \text { se } u \in C_{-}
\end{aligned}
$$

Seja $\mathrm{h} \geq 0$. A aplicação

$$
\left(x_{1}, \ldots, x_{h}\right) \rightarrow x_{h} \otimes \ldots \otimes x_{1} \text { de } M x \ldots x M \rightarrow T^{h}(M)
$$

è obviamente multilinear. 
Portanto, existe uma aplicação linear

$$
\begin{gathered}
\alpha_{h}^{T}: T^{h}(M) \rightarrow T^{h}(M) \text { tal que } \\
\alpha_{h}^{T}\left(x_{1} \otimes \ldots x_{h}\right)=x_{h} \otimes \ldots x_{1} \text { se } x_{1}, \ldots, x_{h} \in M .
\end{gathered}
$$

Seja $\alpha^{\mathrm{T}}$ a aplicação linear de $\mathrm{T}(\mathrm{M})$ sobre ele mesmo que estende todas as aplicações $\alpha_{h}^{T}$. E claro que $\left(\alpha^{T}\right)^{2}$ é identidade.

$$
\begin{aligned}
& \text { Se } x_{1}, \ldots, x_{h}, y_{1}, \ldots, y_{h} \text { estão em } M, \\
& t=x_{1} \otimes \ldots \otimes x_{h}, t^{\prime}=y_{1} \otimes \ldots \otimes y_{k},
\end{aligned}
$$

então temos:

$$
\alpha^{T}\left(t \otimes t^{\prime}\right)=y_{k} \otimes \ldots \otimes y_{1} \otimes x_{h} \otimes \ldots \otimes x_{1}=\alpha^{T}\left(t^{\prime}\right) \otimes \alpha^{T}(t),
$$

o que prova que $\alpha^{T}$ é um anti-automorfismo de $T(M)$.

Este anti-automorfismo deixa os elementos de $T^{\circ}(M)=K$ e $T^{1}(M)=M$ fixados; ele leva sobre eles mesmos os geradores $\mathrm{x} Q \mathrm{x}-\mathrm{Q}(\mathrm{x}) \cdot 1$ de $I$.

Mas I é o conjunto de todos os elementos que são somas de produ tos da forma

$$
t \otimes(x \otimes x-Q(x) .1) \otimes t^{\prime}, \quad c o m t, t^{\prime} \in T(M), \quad x \in M
$$

Isto implica que

$$
\alpha^{\mathrm{T}}(\mathrm{I})=\mathrm{I}
$$

Então $\alpha^{\mathrm{T}}$ define de uma maneira natural uma aplicação linear $\alpha$ : $C(M)+C(M)$, sendo $C(M)=\frac{T(M)}{I}$. E claro que $\alpha \bar{e}$ um anti-automorfismo de $C$, cujo quadrado é a identidade; é chamado o anti-automorfismo principal de $C(M)$.

$$
\alpha^{2}=I_{M}
$$


Definição 1.15. 0 anti-automorfismo $\alpha: c+C$ tal que $\alpha(x)=x$ para todo $x \in M \bar{e}$ chamado anti-automorfismo principal de C.

Temos o seguinte teorema fundamenta1:

Teorema 1.16. "Seja $\varphi$ uma aplicação linear de M numa ālgebra C' sobre K. Su ponhamos que $(\varphi(x))^{2}=Q(x) \cdot 1$ para $x \in M$. Então $\varphi$ pode ser estendido a um homomorfismo $\psi$ de $C$ em $C^{\prime}$. Se $\varphi$ (M) gera $C^{\prime}$, en tão $\varphi(c)=c^{\prime}$.

\section{Prova:}

Seja $C^{\prime}$ uma ālgebra sobre $K$ e $P: M \rightarrow M^{\prime} \underset{\text { s.e. }}{\subset} C^{\prime}$ uma aplicação tal que

$$
(\varphi(x))^{2}=Q(x) \cdot 1 \quad \forall x \in M
$$

Seja $\pi$ a aplicação canônica $\pi: T(M) \rightarrow C=\frac{T(M)}{I}$. Então existe um homomorfismo $\psi: C \rightarrow C^{\prime}$ tal que $\psi(\pi(x))=\varphi(x) \quad \forall x \in M$.

De fato, sabemos que $\varphi$ pode ser estendido a : um homomorfismo $\phi: \mathrm{T}(\mathrm{M}) \rightarrow \mathrm{C}^{\prime}$.

Se $x \in M$, então

$$
\phi(x \otimes x-Q(x) \cdot 1)=(\varphi(x))^{2}-Q(x) \cdot 1=0 .
$$

Isto mostra que o núcleo da $\phi$ contém I (núcleo de $\pi$ ). Então $\phi$ pode ser na forma

$$
\phi=\psi \circ \pi
$$

onde $\psi: C \rightarrow C^{\prime}$ é um homomorfismo com a propriedade exigida. Se $M^{\prime}$ gera $C^{\prime}$, então, temos

$$
\psi(C)=C^{\prime}
$$


Agora construiremos uma ā1gebra $C^{\prime}$.

Seja $E=\Lambda M$ a álgebra exterior sobre $M$, na qual a multiplicação serā representada por $\wedge$.

Sabemos que, sendo $\lambda$ uma função linear em $M$, existe uma anti-de rivação $\delta$ de $\Lambda$ M tal que $\delta \cdot x=\lambda(x) \cdot 1$ para $x \in M, \delta$ é homogênea de grau -1 e $\delta^{2}=0$. Existe uma forma bilinear $B_{0}: M \times M \rightarrow K$ tal que $B_{0}(x, x)=Q(x)$ para todo $\mathrm{x} \in \mathrm{M}$.

Representamos por $\delta_{x}$ a anti-derivação de $\Lambda M$ tal que

$$
\delta_{x} \cdot y=B_{0}(x, y) \quad y \in M
$$

Seja $L_{x}$ o operador $u \rightarrow x \wedge$ da multiplicação à esquerda por $x$ em E; coloca-se

$$
L_{x}^{\prime}=L_{x}+\delta_{x}
$$

Então $x+L_{x}^{\prime}$ é uma aplicação linear $\varphi: M \rightarrow \mathbf{C}$ dos endomorfismos do espaço vetorial $\Lambda \mathrm{M}$.

Mostraremos que vale:

$$
(\varphi(x))^{2}=L_{x}^{\prime 2}=Q(x) \cdot I
$$

onde I è a aplicação identidade.

De fato:

$$
\mathrm{L}_{\mathrm{x}}^{\prime 2}=\mathrm{L}_{\mathrm{x}}^{2}+\mathrm{L}_{\mathrm{x}} \delta_{\mathrm{x}}+\delta_{\mathrm{x}} \mathrm{L}_{\mathrm{x}}+\delta_{\mathrm{x}}^{2}
$$

$$
\begin{aligned}
\text { Temos que } \mathrm{L}_{\mathrm{x}}^{2} & =\delta_{\mathrm{x}}^{2}=0 \mathrm{e} \\
\delta_{\mathrm{x}} \mathrm{L}_{\mathrm{x}} \cdot \mathrm{u} & =\delta_{\mathrm{x}}(\mathrm{x} \wedge \mathrm{u})=\left(\delta_{\mathrm{x}} \mathrm{x}\right) \wedge \mathrm{u}-\mathrm{x} \wedge\left(\delta_{\mathrm{x}} \mathrm{u}\right)= \\
& =\mathrm{Q}(\mathrm{x}) \cdot \mathrm{u}-\mathrm{L}_{\mathrm{x}} \cdot \delta_{\mathrm{x}} \mathrm{u}
\end{aligned}
$$




$$
\begin{gathered}
\text { Portanto, } L_{x} \delta_{x}+\delta_{x} L_{x}=Q(x) \cdot I \quad e \\
L_{x}^{\prime 2}=Q(x) \cdot I
\end{gathered}
$$

Esta igualdade implica que existe um homomorfismo $\psi$ de $\mathrm{C}$ em tal que $\psi(\pi(x))=L_{x}^{\prime}$ para $x \in M$.

Verifica-se que $\varphi: x \rightarrow L_{x}^{\prime} \bar{e}$ um isomorfismo de $M$, pois:

$$
L_{x}^{\prime} \cdot 1=x \wedge 1+\delta_{x} \cdot i=x,
$$

desde que $\delta_{x} \cdot 1=0$, se $x \in M$ e temos o núcleo de $\varphi$ igual a zero.

Segue que $\pi$ induz um isomorfismo de $M$ em $C$. Poderemos portanto identificar os elementos de M com suas imagens em $C$ pela aplicação $\varphi$ e M será considerado como um subespaço de C. Temos

$$
x^{2}=Q(x) \cdot 1 \quad \text { se } \quad x \in M
$$

$$
\begin{aligned}
& \text { Aplicando (3) a } x, y e x+y \text { (onde } x, y \in M) \text { e usando o fato } \\
& Q(x+y)-Q(x)-Q(y)=B(x, y) \text { obtemos } \\
& x y+y x=B(x, y) \cdot 1, \quad x, y \in M
\end{aligned}
$$

Enunciaremos as propriedades fundamentais da älgebra de Clifford que serão utilizadas posteriormente.

Proposição 1.17: [8] "Seja $\left\{x_{1}, \ldots, x_{m}\right\}$ base de M. Se $\sigma=\left(i_{1}, \ldots, i_{h}\right)$ é uma seq Uência estritamente crescente de inteiros $i_{1}, \ldots, i_{h}$ entre 1 e m, seja $P(\sigma)$ o produto $x_{i_{1}} \cdots x_{i_{h}}$ em C. Então os elementos $P(\sigma)$ formam uma base de $C$, que $\bar{e}$ de dimensão $2^{\text {m }}$ ". 
Proposição 1.18. [8] "Seja a notação de 1.16 , se $\varphi(M)$ gera $C^{\prime}$ e $C$ ' é de dimensão $\geq 2^{\mathrm{m}}$, então $\psi$ é um isomorfismo de $C$ com $C^{\prime} . "$

Proposição 1.19. [8] "Seja N um subespaço de M. Então a subälgebra de C gerada por $\mathrm{N}$ é isomorfa à älgebra de Clifford da restrição de $Q$ a $\mathrm{N}^{\prime \prime}$.

Retornamos ao homomorfismo $\psi: C \rightarrow C$ considerando acima. Coloca-se

$$
\theta(u)=\psi(u)(1) \quad \text { para } \quad u \in C
$$

Então $\theta$ é uma aplicação linear de $C(M)$ em $\Lambda M$ e desde que, dim $C(M)=2^{m}=\operatorname{dim} \cdot M, \theta \bar{e}$ um isomorfismo linear, que coincide com a identidade em K 1 e em M. Geralmente, identificaremos o espaço vetorial básico de C(M) com o espaço vetorial de $\Lambda$, através de $\theta$. Entretanto, devemos sempre considerar, que essa identificação depende da escolha de uma forma bilinear $B_{0}$ tal que $B_{0}(x, x)=$ $=Q(x)$.

Portanto

$$
C(M) \cong \Lambda M \quad \text { (de espaços vetoriais) }
$$

Finalmente, enunciaremos um teorema de maior importância, que de termina a estrutura da älgebra de clifford para espaços vetoriais de dimensão par.

Tearema 1.21. [8] "Seja M um espaço vetorial de dimensão $2 r$ e Q de posto m. Então a ālgebra de clifford $C$ de $Q \bar{e}$ uma álgebra central simples. Além disso, se $Q$ é de indce $r$, então Ćé isomorfa a álgebra de todas as matrizes de ordem $2^{r}$ com coeficiente em $K^{\prime \prime}$.

Apliquemos os resultados obtidos, na determinação da ālgebra de Clifford do espaço $M=E \oplus \mathrm{E}^{*}$ definido anteriormente, em relação à forma quadrá tica canônica. 
A álgebra de Clifford $C$ de $M$ em relação à forma quadrática $Q$, é a ālgebra $C_{M}=\frac{T(M)}{I}$, onde $I \bar{e} \circ$ ideal gerado pelo conjunto $S= \begin{cases}z \otimes & Q(z) \cdot 1\end{cases}$ para $z \in M\}$.

Se $z=x+y^{\prime} \in M, S$ pode ser escrito

$$
S=\left\{\left(x+y^{\prime}\right) \otimes\left(x+y^{\prime}\right)-y^{\prime}(x) \cdot 1 \quad x \in E, y^{\prime} \in E^{*}\right\}
$$

Então, considerando-se M como subespaço de C, fica:

$$
x^{2}+x \cdot y^{\prime}+y^{\prime} x+y^{\prime 2}=\left[y^{\prime}(x)\right] \cdot 1 \quad x \in E, y^{\prime} \in E^{*}
$$

o que implica que

$$
x \cdot y^{\prime}+y^{\prime} x=\left\langle x, y^{\prime}\right\rangle \cdot 1, \quad x \in E, y^{\prime} \in E^{*}
$$

pois:

$$
x^{2}=Q(x)=0 \text { e } y^{\prime 2}=Q\left(y^{\prime}\right)=0
$$

considerando-se que

$$
Q(E)=0 \text { e } Q\left(E^{*}\right)=0 \text {. }
$$

Se considerarmos a base de $E\left\{x_{1}, \ldots, x_{n}\right\}$ e a base dual $\left\{y_{1}^{\prime}, \ldots, y_{n}^{\prime}\right\}$ de $E^{*}$, vale:

$$
x_{i} y_{j}^{\prime}+y_{j}^{\prime} x_{i}=\left\langle x_{i}, y_{j}^{\prime}\right\rangle \cdot 1=\delta_{i j} \cdot 1
$$

que é a tābua da multiplicação da ālgebra $G_{n}$. 
Lembramos que a forma bilinear associada à $Q$ é dada por

$$
B\left[\left(x, x^{\prime}\right),\left(y, y^{\prime}\right)\right]=\left[\left(x^{\prime}(y)+y^{\prime}(x)\right]\right.
$$

ou

$$
B_{0}\left[\left(x, x^{\prime}\right),\left(y, y^{\prime}\right)\right]=\frac{1}{2}\left[x^{\prime}(y)+y^{\prime}(x)\right]
$$

Considerando-se que a restrição da forma quadrätica canônica Q, aos subespaços $E$ e $E^{*}$ de $M$, é igual a forma nula, isto é,

$$
Q_{\mid E}=0 \quad \text { e } \quad Q_{\mid E} *=0,
$$

conclui-se que as älgebras de Clifford das restrições de $Q$ a $E$ e a $E^{*}$, são iso morfas às álgebras exteriores de $E$ e $E^{*}$.

Em símbolos,

$$
C_{(E)} \cong \Lambda E \quad \text { e } \quad C_{\left(E^{*}\right)}=\Lambda E^{*}
$$

o que é equivalente a afirmar,

$$
\begin{array}{ll}
x^{2}=x \wedge x=0 & \forall x \in E \\
x^{\prime 2}=x^{\prime} \wedge x^{\prime}=0 & \forall x^{\prime} \in E^{*}
\end{array}
$$

Além disso, é importante destacar que, sendo a dimensão do espa ço par $=2 n$ e $Q$ de índice $n, C_{M} \bar{e}$ isomorfa à álgebra de todas as matrizes de ordem $2^{\mathrm{n}}$, com coeficientes em $\mathrm{K}$ e $\overline{\mathrm{e}}$ uma álgebra central simples(aplicando-se o Teo rema 1.21 ).

A seguir, daremos a definição e propriedades de uma álgebra que está intimamente relacionada com a álgebra de Clifford: é a chamada Álgebra de Wey 1.

Seja K um corpo comutativo de característica nula e $\mathrm{M}$ um K-espa ço vetorial. 
Definição 1.22. Uma forma bilinear $f: M \times M \rightarrow K$ é anti-simétrica se e somente se

(a) $\quad f(x, y)=-f(y, x) \quad x, y \in M$

Esta condição é equivalente a:

(b) $\quad f(x, x)=0 \quad x \in M$

(pois $\mathrm{K}$ tem característica nula)

Definição 1.23. A Âlgebra de Weyl de um espaço vetorial M, $\mathrm{C}_{\mathrm{S}}(\mathrm{M}, \mathrm{f})$ è a álgebra quociente da ālgebra tensorial T(M) pelo ideal bilateral gerado pelos elementos.

$$
z \otimes w-w \otimes z-f(z, w) \quad z, w \in M
$$

onde f è uma forma bilinear anti-simétrica.

Muitas das propriedades das ālgebras de Clifford tem analogia com as da álgebra de Weyl; por exemplo toda forma bilinear B sobre $M$ tal que $B(a, b)-B(b, a)=f(a, b)$ se $a, b \in M$, determina um isomorfismo $J_{B}$ do espaço vetorial $C_{S}(M, f)$ sobre o espaço vetorial subjacente à álgebra simétrica SM.

Pode-se definir uma extensão desta álgebra.

Definição 1.24. Seja F uma forma bilinear anti-simétrica de "ordem" maximal sobre M (forma simplética). A ālgebra de Clifford simplética é a ālgebra quociente da álgebra tensoria1 T(M) pelo ideal bilateral gerado pelos elementos

$$
z=w-w a z-F(z, w) \quad z, w \in M
$$

Notação: $C_{S}(M, F)$

A álgebra $C_{S}(M, F)$ é associativa e é única a menos de isomorfismo (propriedade universal). 
Teorema 1.25. [12] "Seja A uma älgebra associativa sobre K, e u uma aplicação li near de $M$ em $A$ tal que

$$
u(x) \cdot u(y)-u(y) \cdot u(x)=F(x, y) \quad x, y \in M
$$

então existe um homomorfismo $\bar{u}$ e um só de $C_{S}(M, F)$ em A tal que $u=\vec{u} \circ \rho_{F}$, onde $\rho_{F} \quad \bar{e}$ a aplicação canônica $\rho_{F}: T(M) \rightarrow C_{S}(M, F) "$.

Teorema 1.26. [12] "A älgebra $\mathrm{C}_{\mathrm{S}}(\mathrm{M}, F)$ é linearmente isomorfa à ālgebra simétrica $\mathrm{SM}^{\prime \prime}$.

Retomamos o espaço vetorial $\mathrm{M}=\mathrm{E} \oplus \mathrm{E}^{*}$, definido anteriormen

te.

Seja F: $M \times M \rightarrow K$ a forma bilinear anti-simétrica

$$
F(z, w)=F\left(\left(x, x^{\prime}\right),\left(\left(y, y^{\prime}\right)\right)=\left[y^{\prime}(x)-x^{\prime}(y)\right]\right.
$$

Então, a multiplicação de $C_{S}(M, F)$ é dada por

$$
\begin{gathered}
z \cdot w-w \cdot z=F(z, w) \quad z, w \in M \\
\left(x+x^{\prime}\right)\left(y+y^{\prime}\right)-\left(y+y^{\prime}\right)\left(x+x^{\prime}\right)=\left[y^{\prime}(x)-x^{\prime}(y)\right] \cdot 1 \quad x, y \in E, x^{\prime}, y^{\prime} \in E^{*}
\end{gathered}
$$

ou

$x y^{\prime}+x^{\prime} y-y x^{\prime}-y^{\prime} x=\left[y^{\prime}(x)-x^{\prime}(y)\right] \cdot 1$

Fazendo $z=x \in E$, a igualdade (7) fica:

$$
x y^{\prime}-y^{\prime} x=\left[y^{\prime}(x)\right] \cdot 1 \quad x \in E, y^{\prime} \in E^{*}
$$

Se $\left\{x_{i}\right\}_{1 \leq i \leq n}$ é base de $E$ e $\left\{y_{j}^{\prime}\right\}_{1 \leq j \leq n}$ a base dual de $E^{*}$, temos:

$$
x_{i} y_{j}^{\prime}-y_{j}^{\prime} x_{i}=\delta_{i j} \cdot 1
$$


A álgebra $C_{S}(M, f)$ definida por estas igualdades constitui a ā1gebra $L_{n}$, definida por Schemberg.

E importante ressaltar que a ālgebra $C_{S}(M, F)$ restrita a $E$ é iso morfa à älgebra simétrica sobre $\mathrm{E}$ e a älgebra $\mathrm{C}_{\mathrm{S}}(\mathrm{M}, \mathrm{F})$ restrita ao subespaço $\mathrm{E}^{*}$ de $M$ é isomorfa à ālgebra simétrica sobre $E^{*}$.

Em símbolos, na älgebra $\mathrm{C}_{\mathrm{S}}(\mathrm{M}, \mathrm{F})$ vale:

$$
\begin{array}{ll}
x y=y x & \forall x, y \in E \\
x^{\prime} y^{\prime}=y^{\prime} x^{\prime} & \forall x^{\prime}, y^{\prime} \in E^{*}
\end{array}
$$

Portanto, us ando a notação de Schemberg [29]; concluímos que as álgebras $G_{\mathfrak{n}}$ e $L_{\mathfrak{n}}$ são extensões das ālgebras exterior e simétrica de vetores contravariantes.

Usando a definição de plano hiperbólico e observando-se que det $M=-1$ (em relação à forma bilinear $B_{O}$ ), analisaremos a decomposição do espaço $M$ em planos hiperbólicos. (det $M=-1$ se $n=\operatorname{dim} E$ é ímpar).

Teorema 1.27. [13]"Seja (M,B) um espaço vetorial munido de uma forma B simétrica ou anti-simétrica não-degenerada. Se $S$ é um espaço totalmente singular de $M$, de base dada $\left(x_{i}\right)_{1 \leq i \leq r}$, existe um sub-espaço $S^{\prime}$ totalmente singular, de mesma dimensão que $S$, $e$ uma base $\left(x_{j}^{\prime}\right)_{1 \leq j \leq r}$ de $S^{\prime}$ tais que:

1) Para cada $j, 1 \leq j \leq r, x_{j}$ e $x_{j}^{\prime}$ formam uma base de um plano regular $H_{j}\left(p l\right.$ ano hiperbölico) e $B\left(x_{i}, x_{j}^{\prime}\right)=1$ se $i=j$, 0 se $i \neq j$.

2) A soma $S+S^{\prime}$ é direta, e é um sub-espaço regular de $(M, B)$, soma direta ortogonal dos $p l$ anos $H_{j}$ (planos hiperbólicos):

$$
\mathrm{S} \oplus \mathrm{S}^{\prime}=\mathrm{H}_{1} \perp \mathrm{H}_{2} \perp \cdots \perp \mathrm{H}_{\mathrm{r}}
$$

decomposição dita hiperbōlica de $S \oplus S^{\prime} . "$ 
Proposição 1.28. [13] "Seja (M,B) um espaço vetorial de dimensão $2 n$ munido de uma for ma bilinear B, simétrica ou anti-simétrica, não degenerada, cuja aplicação linear associada à direita, bijeção de $M$ sobre $M^{*}$, e representada $\rho,\langle x, \rho(y)\rangle=B(x, y)$. Suponhamos que existe um sub-espaço $S$ totalmente singular de dimensão $n$, onde uma base $\left(x_{i}\right)_{1 \leq i \leq n} \bar{e}$ dada; todo outro espaço totalmente singular $S^{\prime}$, de dimensão $n$, suplementar de $S\left(S \cap S^{\prime}=0\right)$ é identificado ao dual de $S$ e possui uma base $\left(x_{j}^{\prime}\right)_{1 \leq j \leq n}$ tal que $B\left(x_{i}, x_{j}^{\prime}\right)=\delta_{i j}$, a restrição de $\mathrm{B}$ à $\mathrm{S} \times \mathrm{S}^{\prime}$ se identificando ao "colchete" de dualí dade".

No espaço vetorial $\mathrm{M}=\mathrm{E} \oplus \mathrm{E}^{*}$ temos a forma $\mathrm{B}$ simétrica e a for ma $B^{\prime}$ anti-simétrica:

$$
\begin{array}{ll}
B\left(x+x^{\prime}, y+y^{\prime}\right)=\left\langle x, y^{\prime}\right\rangle+\left\langle y, x^{\prime}\right\rangle & x, y \in E \\
B^{\prime}\left(x+x^{\prime}, y^{\prime}+y^{\prime}\right)=\left\langle x, y^{\prime}\right\rangle-\left\langle y, x^{\prime}\right\rangle & x^{\prime}, y^{\prime} \in E^{*}
\end{array}
$$

A restrição de $B$ e $B^{\prime}$ à $E \times E^{*}$ è a forma bilinear canônica.

$$
B\left(x, y^{\prime}\right)=B^{\prime}\left(x, y^{\prime}\right)=\left\langle x, y^{\prime}\right\rangle
$$

Conclui-se então, que, em $\mathrm{M}, \mathrm{E}^{*}$ pode ser identificado a um subespaço $E^{\prime}$ totalmente singular de dimensão $n$, onde uma base $\left(x_{i}^{\prime}\right)_{1 \leq i \leq n}$ é dada e a soma $E \oplus E^{\prime}$ pode ser escrita como a soma direta ortogonal dos planos hiperbólí $\cos H_{i}:(1 \leq i \leq n)$

$$
E \oplus E^{\prime}=H_{1} \perp H_{2} \perp \cdots \perp H_{n}
$$

o que equivale a afirmar que $M$ é um espaço hiperbólico.

Reciprocamente, um espaço hiperbólico $(M, Q)$ de dimensão $2 n$ pode ser decomposto em soma direta de dois subespaços totalmente singular maximais $\mathrm{E}$ e $E^{\prime}$, de dimensão $n$, colocados em dualidade pela forma bilinear associada à 
$Q(M, Q)$ é isomorfo ao espaço quadrático $E \oplus E^{*}$, munido da forma bilinear simétrica

$$
B\left(x+x^{\prime}, y+y^{\prime}\right)=\left\langle x, y^{\prime}\right\rangle+\left\langle y, x^{\prime}\right\rangle
$$

Teorema 1.29. [13]"Seja (M,Q) um espaço quadrático sobre um corpo ordenado. Todos os subespaços estritamente positivos maximais tem mesma dimen$s$ ão $p$, todos os subespaços estritamente negativos maximais tem mesma dimensão $q$. 0 par de inteiros $(p, q)$ é chamado o índice de inércia de $Q$, que é de "posto" $p+q . S S e Q(z)=\sum_{i=1}^{n}\left(a_{i}\right)^{2} x_{i}$ em uma base ortogonal de $M,\left(z=\sum_{i} a_{i} x_{i} \in M\right.$ e $\left.Q\left(x_{i}\right)=x_{i} 1 \leq i \leq n\right), \quad o$ número de coeficientes $X_{i}$ estritamente positivo é igual à $p$, o número de coeficientes $X_{i}$ estritamente negativo é igual à q e es tes nümeros são os mesmos para toda base ortogonal".

Esta forma pode se escrever em uma base oxtogonal conveniente:

$$
Q(z)=\left(x_{1}\right)^{2}+\left(x_{2}\right)^{2}+\ldots+\left(x_{p}\right)^{2}-\left(x_{p+1}\right)^{2}-\ldots-\left(x_{p+q}\right)^{2}
$$

Definição 1.30. Um espaço quadrático real regular $(M, Q)$ è chamado espaço euclidiano se $Q$ é estritamente positiva, anti-euclidiano se $Q$ é es tritamente negativa, pseudo-euclidiano se $Q \bar{e}$ de índice $(p, q)$ $\operatorname{com} \mathrm{p}>0$ e $\mathrm{q}>0$.

Verifica-se que o espaço $M=E \oplus E^{*}$ sobre o corpo $\mathbb{R}$ é um espaco pseudo-euclidiano com índice de inércia igual a $(n, n)$. 


\section{CAPÍTULO II}

\section{GRUPO DE CLIFFORD}

Neste capítulo são válidas as notações já utilizadas anterior mente.

$Q$ representa a forma quadrática sobre um espaço vetorial $M$ de dimensão m sobre um corpo $K, B$ é a forma bilinear associada a $\dot{Q}$ e supõe-se que ela é não degenerada; $C$ é a âlgebra de Clifford de $Q$ e $G$ o grupo ortogonal de Q.

Definição 2.1. Chama-se grupo de Clifford de $G$ e representa-se por $\Gamma$, o grupo de elementosinversíveis $s$ de $C$ tal que $\mathrm{s} \mathrm{s}^{-1} \in \mathrm{M}$ para todo $x \in M$.

Se $s \in \Gamma$, representa-se por $\chi(s)$ o automorfismo 1 inear $x \rightarrow s \mathrm{xs}^{-1}$ de $M$ e $\chi: s \rightarrow \chi(s)$ é uma representação linear de $\Gamma$, chamada a representação vetorial de $\Gamma$.

Seja $s \in \Gamma$. Então temos, para $x \in M$,

$$
Q\left(s \times s^{-1}\right)=\left(s \times s^{-1}\right)^{2}=s x^{2} \cdot s^{-1}=Q(x) \cdot 1
$$

Isto implica que $X$ leva $\Gamma$ no grupo ortogonal $G$ de $Q$.

Teorema 2.2. [8] Se a dimensão do espaço vetorial M é par, então

$$
\chi(\Gamma)=G .
$$

Seja $\mathrm{H}$ um hiperplano de $M$ cujo conjugado contēm um vetor não sin gular z. Para $x \in M$, define-se

$$
\tau_{z}: x=x-[Q(z)]^{-1} \cdot B(x, z) \cdot z
$$


$\tau_{2} \quad \bar{e}$ um endomorfismo de $M$, que pertence ao grupo ortogonal $G$, e é chamada a simetria em relação a H.

Tearema 2.3. "Se $x$ é um elemento não singular de $M$, então $x \in \Gamma$ e $\chi(x)$ é a aplicação $y \rightarrow-\tau y$, onde $\tau \bar{e}$ a simetria em relação ao hiperplano conjugado de $\mathrm{Kx}^{\prime \prime}$.

\section{Prova:}

Seja x um elemento não singular de M. Então $x$ é inversível, e $\mathrm{x}^{-1}=[\mathrm{Q}(\mathrm{x})]^{-1} \cdot \mathrm{x}$.

$$
\begin{aligned}
& \text { Temos } x y+y x=B(x, y) 1 \text { para } y \in M \\
& x y x^{-1}+y=B(x, y) \cdot x^{-1} \\
& x y x^{-1}=[Q(x)]^{-1} \cdot B(x, y) x-y=-\tau \cdot y
\end{aligned}
$$

donde

$$
x(x) \cdot(y)=-\tau_{x} y
$$

onde $\tau \bar{e}$ a simetria em relação ao conjugado de $k . x$.

$$
\text { Segue que } x \in \Gamma \text { e } \chi(x)=-\tau \text {. }
$$

Teorema 2.4. "Seja $\mathrm{Z}^{*}$ ○ grupo multiplicativo dos elementos inversíveis do cen tro $Z$ de $C$; então $Z^{*}$ é o núcleo de $X$ e, exceto no caso que $K$ é um corpo com 2 elementos, $\operatorname{dim} M=4$ e $Q \bar{e}$ de indice $2, z^{*} U(\Gamma \cap M)$ é um conjunto de geradores do grupo $\Gamma^{\prime \prime}$.

\section{Prova:}

Se $s \in z^{*}, \chi(s)(x)=x$ para $x \in M$ e portanto $z^{*} \subset \Gamma$, e é óbvio que $Z^{*}$ está no núcleo de $\chi$. 
Reciprocamente, se $s \in \Gamma, \chi(s)=I$, então $s$ comuta com todo ele mento de $M$, e $s \in Z \cap \Gamma=Z^{*}$.

Excluímos o caso excepcional mencionado no enunciado. Toda operação de $G$ pode ser escrita como um produto $\tau_{1} \ldots \tau_{h}$ de simetrias em relação aos hiperplanos cujos conjugados contém vetores não singulares $x_{1}, \ldots, x_{h}$.

Seja $\zeta$ a aplicação $x+-x(x \in M)$, e $\zeta^{\prime}$ o automorfismo de $C$ que es tende $\zeta$. Vale $\zeta^{\prime}(u)=-u$ para $u \in C_{-}$.

Então, desde que $\zeta^{\overline{2}}=1$, temos $\sigma=\zeta^{h} \chi\left(x_{1} \ldots x_{h}\right)$

Se mé ímpar, então temos det $\tau_{i}=+1$, det $\zeta=-1$, e, se $\sigma \in \mathrm{G}^{+}$, então temos $h \equiv 0(\bmod 2)$ e $\sigma=\chi\left(x_{1} \ldots x_{h}\right)$; se $\sigma=\chi(s), s \in \Gamma$, então $s=$ $=s_{0} s_{1} \cdots s_{h} \operatorname{com} s_{0} \in Z^{*}$.

Se mé par, então $\zeta$ pertence ao grupo gerado por $\Gamma \cap \mathrm{M}$. De fato, seja $\left(y_{1}, \ldots, y_{m}\right)$ uma base de $M$ composta de vetores mutuamente ortogonais. En tão

$$
\begin{aligned}
& x\left(y_{i}\right)\left(y_{j}\right)=-y_{j} \text { se } i \neq j \\
& x\left(y_{i}\right)\left(y_{i}\right)=y_{i} \cdot
\end{aligned}
$$

donde

$$
\zeta=x\left(y_{1} \cdots y_{m}\right) .
$$

Então, segue do mesmo modo que acima que todo $s \in \Gamma$ pertence ao grupo gerado por $\Gamma \cap \mathrm{M}$ e $\mathrm{z}^{*}$.

Teorema 2.5. [8] "Todo $s \in \Gamma$ pode ser escrito na forma $z s$ ', onde s está no cen tro de $C$ e s' é um elemento de $\Gamma$ que è par ou ímpar. Se mé par, $s$ é ou par ou impar.

Representa-se por $\Gamma^{+}$o grupo de elementos pares de $\Gamma$.

Teorema 2.6. [8] Toda operação de G pertence ao grupo G' gerado pelas simetrias em relação aos hiperplanos cujos conjugados contém vetores não singulares.

Retornemos ao espaço quadrático $M=E \oplus E^{*}$, onde $E$ é um K-espaço vetorial de dimensão $\mathrm{n}$, com a forma quadrática canônica $\mathrm{Q}$.

Então $\Gamma$ é gerado pelo conjunto $K^{*} U(\Gamma \cap M)$ onde

$$
\Gamma \cap M=M_{1}=\{z \in M \text { t.q. } Q(z) \neq 0\}
$$


ou

$$
M_{1}=\left\{z=\left(x+x^{\prime}\right) \in M \quad t \cdot q \cdot\left\langle x, x^{\prime}\right\rangle \neq 0\right\}
$$

Portanto $z \in M_{1} \Longrightarrow z=x+x^{\prime}$ t.q. $x \neq 0$ e $\quad x^{\prime} \neq 0$.

Verifica-se também aplicando-se [2.5] neste caso particular que um elemento $s \in \Gamma \bar{e}$ da forma

$$
\mathrm{s}=\mathrm{k} \cdot \mathrm{s}_{1} \ldots \mathrm{s}_{\mathrm{h}} \quad \mathrm{k} \in \mathrm{K}^{*}
$$

e $s_{i}=x_{i}+x_{i}^{\prime}, 1 \leq i \leq h \quad$ onde $\quad\left\langle x_{i}, x_{i}^{\prime}\right\rangle \neq 0$ e $h$ pode ser par ou impar.

Seja $\sigma$ uma operação do grupo.G. Como $\operatorname{dim} M=2 n \bar{e}$ par, existe s $\in \Gamma$ t.q. $\chi(s) \equiv \sigma$.

Aplicando 2.6., vemos que:

se $\sigma \in G \Longrightarrow$ existe $s=k_{1} \ldots s_{h}$ t.q. $X(s) \equiv \sigma$ e para $z \in M$

$$
\begin{aligned}
& \chi(s)(z)=s z s^{-1}=\left(s_{1} \ldots s_{h}\right) z\left(s_{1} \ldots s_{h}\right)^{-1}= \\
& =-\tau_{s_{1}} \cdot-\tau_{s_{2}} \cdots-\tau_{s_{h}}(z)
\end{aligned}
$$

onde $\tau_{\dot{s}_{i}}$ é a simetria em relação ao vetor $s_{i}$ e $s_{i}$ é não-singular.

Determinemos as operações do grupo $G$ de $M=E \oplus E^{*}$

$G_{M}=\left\{F: M \rightarrow M\right.$ automorfismos lineares t.q. $\left.Q\left(F\left(x, x^{\prime}\right)\right)=\left\langle x, \dot{x}^{\prime}\right\rangle,\left(x, x^{\prime}\right) \in M\right\}$

Seja $B: E \times E \rightarrow K$ uma forma bilinear. Para todo $x \in E$, temos a

forma linear em E

$$
u(x): y \rightarrow B(x, y)
$$

e $\mathrm{u}: \mathrm{E} \rightarrow \mathrm{E}^{*}$ é a aplicação linear canônicamente associada à B.

Observemos que se B é não-degenerada, u é injetora e como dim $E=$ $=\operatorname{dim} E^{*}, u \bar{e}$ um isomorfismo de $E$ sobre $E^{*}$. 
Seja $\mathrm{F}$ um K-espaço vetorial.

Definição 2.7. Chama-se transposta de uma aplicação linear $\mathrm{u}: \mathrm{E} \rightarrow \mathrm{F}$, e represen

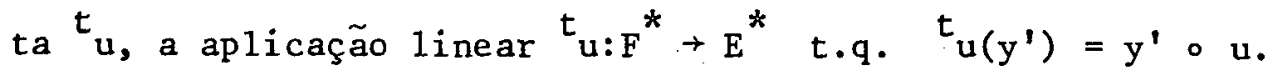

Valem as propriedades:

(a) $\left\langle u(x), y^{\prime}\right\rangle=\left\langle x,{ }^{t} u\left(y^{\prime}\right)\right\rangle \quad x \in E, y^{\prime} \in F^{*}$

(b) Se $u: E \rightarrow F$ e $v=F \rightarrow H$ (H é um $K$-espaço vetoria1)

$$
t_{(v \circ u)}=t_{u \circ} t_{v}
$$

(c) ${ }^{t}\left({ }^{t} u\right)=u$

(d) Se u:E $\rightarrow F$ um isomorfismo, então ${ }^{t} u: F^{*} \rightarrow E^{*}$ é um iso morfismo, e

$$
t_{u}^{-1}=\left(t_{u}\right)^{-1}
$$

Definição 2.8. Seja u:E $\rightarrow$ F um isomorfismo; chama-se isomorfismo contragradien te de u (ou aplicação contragradiente de u) e representa-se $\stackrel{\mathrm{u}}{\text {, }}$ a transposta do isomorfismo recíproco de u (igual ao isomorfismo recíproco de ${ }^{t} u$ ), isto $e$,

$$
\mathrm{v}={ }^{t}\left(u^{-1}\right)=\left({ }^{t} u\right)^{-1}
$$

0 isomorfismo $\mathrm{U}$ é então definido pela identidade

$$
\left\langle\mathrm{u}(\mathrm{x}), \mathrm{v}\left(\mathrm{x}^{\prime}\right)\right\rangle=\left\langle\mathrm{x}, \mathrm{x}^{\prime}\right\rangle \quad \mathrm{x} \in \mathrm{E}, \mathrm{x}^{\prime} \in \mathrm{E}^{*}
$$

e temos

$$
\stackrel{\mathrm{V}}{\mathrm{u}})=\mathrm{u}
$$

isto é, u é o isomorfismo contragradiente de $\stackrel{\mathrm{v}}{\mathrm{u}}$. 
Proposição 2.9. Existe um automorfismo $F: M \rightarrow M$, que pertence ao grupo ortogonal de M e troca (entre si) os s.e. totalmente singulares maximais $E$ e $E^{*}$ de $M$.

\section{Prova:}

Seja B:E $\times \mathrm{E} \rightarrow \mathrm{K}$ e $\mathrm{U}: \mathrm{E} \rightarrow \mathrm{E}^{*}$ a aplicação linear associada a B. (B não-degenerada) e $\stackrel{\mathrm{U}}{\mathrm{u}}: \mathrm{E}^{*} \rightarrow \mathrm{E}$. (u é um isomorfismo).

Podemos então definir a aplicação

$$
\begin{gathered}
F: M \rightarrow M \quad t . q . \\
F\left(x, x^{\prime}\right)=\left(u(x), \stackrel{v}{u}\left(x^{\prime}\right)\right) \quad\left(x, x^{\prime}\right) \in M
\end{gathered}
$$

(pode-se também escrever $F\left(x, x^{\prime}\right)=\left(\stackrel{v}{u}\left(x^{\prime}\right), u(x)\right)$.

Obviamente, F.é linear e é um isomorfismo.

F troca os subespaços totalmente singulares maximais de $M$, E e

$E^{*}$, pois:

$$
\begin{aligned}
& \text { Se } x \in E \underset{\text { s.e. }}{\subset} M \\
& \qquad F(x)=F(x, 0)=(u(x), 0) \in E^{*} \Longrightarrow F(E)=E^{*} ;
\end{aligned}
$$

se $x^{\prime} \in E^{*} \subset M$

$$
F\left(x^{\prime}\right)=F\left(0, x^{\prime}\right)=\left(\stackrel{V}{U}\left(x^{\prime}\right), 0\right) \in E \Longrightarrow F\left(E^{*}\right)=E
$$

$F \in$ grupo ortogonal de $M$, pois se $z=\left(x, x^{\prime}\right) \in M$,

$$
\mathrm{Q}(\mathrm{F}(z))=\mathrm{Q}\left(\mathrm{F}\left(\mathrm{x}, \mathrm{x}^{\prime}\right)\right)=\left\langle\mathrm{U}\left(\mathrm{x}^{\prime}\right), \mathrm{u}(\mathrm{x})\right\rangle=\left\langle\mathrm{x}, \mathrm{x}^{\prime}\right\rangle=\mathrm{Q}(\mathrm{z})
$$

Conclui-se que:

a partir de uma função bilinear $B: E \times E \rightarrow K$ (não degenerada), temos um isomorfís mo $\mathrm{u}: \mathrm{E} \rightarrow \mathrm{E}^{*}$, e o isomorfismo contragradiente $\mathrm{U}: \mathrm{E}^{*} \rightarrow \mathrm{E}$. 
Define-se então um automorfismo de $M, F\left(\left(x, x^{\prime}\right)\right)=\left(U_{U}^{V}\left(x^{\prime}\right), u(x)\right)$ que conserva a forma quadrática e troca entre si os s.e. totalmente singulares maximais $E$ e $E^{*}$, isto equivale a afirmar que $F \in G$ (grupo ortogonal de $Q$ ).

Reciprocamente, dado o grupo ortogonal $G$ de $Q$, os s.e. totalmen te singulares maximais deM, $\mathrm{E}$ e $\mathrm{E}^{*}$ são permutados transitivamente entre si por operações de $G$.

\section{Então}

$$
F \in G \Longrightarrow F(E)=E^{*} \text { e } F\left(E^{*}\right)=E ;
$$

define-se então $u: E \rightarrow E^{*}$ isomorfismo e uma forma bilinear não-degenerada (simétrica)

$$
B: E \times E \rightarrow K \quad t \cdot q \cdot u(x)(y)=B(x, y), \quad x, y \in E .
$$

B é simētrica se e somente se $u=$

Estabelece-se então uma correspondência entre as operações de G que trocam os s.e. totalmente singulares $E$ e $E^{*}$ de $M$ e os espaços quadräticos $E$ (espaço vetorial de dimensão finita $\underline{n}$ munido de uma forma bilinear $\mathrm{B}$ e uma forma quadrātica Q).

Teorema 2.10 [8] Sejam E e E' s.e. totalmente singulares de dimensãon. Então existe

$$
s \in \Gamma \quad \text { t.q. } \quad s \mathrm{Es}^{-1}=\mathrm{E}^{\prime}
$$

Teorema $2.11[8]$ Todos os subespaços totalmente singulares maximais tem a mesma dimensão e são permutados transitivamente entre eles por operações de G. 
Portanto, existe

$s \in \Gamma \quad$ t.q. $X(s) \equiv F$,

onde F é o automorfismo da Proposição 2.9 e

$$
X(s) \cdot(E)=E^{*}, X(s)\left(E^{*}\right)=E
$$

Quais são as outras aplicações $F: M \rightarrow M$ que pertencem a $G$ ? Seja

$$
F_{1}: M \rightarrow M \quad \text { t.q. } \quad F_{1}(z)=\lambda z \quad z \in M .
$$

\section{Então se}

$$
z=\left(x, x^{\prime}\right)
$$

temos

$$
Q(\lambda z)=\left\langle\lambda x, \quad \lambda x^{\prime}\right\rangle=\lambda^{2}\left\langle x, x^{\prime}\right\rangle=\left\langle x, x^{\prime}\right\rangle
$$

se e somente se

$$
\lambda^{2}=1 \Longrightarrow \lambda= \pm 1 \text {. }
$$

Portanto

$$
\text { I e }-I \in G \text {. }
$$

Existem outros automorfismos de $M$, que pertencem ao grupo ortogonal G.

$$
\text { Seja }
$$

$$
u^{\prime}: E \rightarrow E
$$

um isomorfismo linear; a transposta ${ }^{t} \mathrm{u}^{\prime}$ é um isomorfismo de $\mathrm{E}^{*}$ sobre $\mathrm{E}^{*}$ e pode-se definir o isomorfismo contragradiente

$$
\stackrel{\mathrm{u}}{\mathrm{u}}^{\prime}=\left({ }^{\mathrm{t}} \mathrm{u}^{\prime}\right)^{-1}: \mathrm{E}^{*} \rightarrow \mathrm{E}^{*}
$$


e o automorfismo linear

$$
F^{\prime}: M \rightarrow M \quad \text { t.q. } \quad F^{\prime}\left(x, x^{\prime}\right)=\left(u^{\prime}(x), \breve{u}^{\prime}\left(x^{\prime}\right)\right)
$$

Proposição 2.12. Além da identidade, existe um automorfismo $F^{\prime}: M \rightarrow M$, que perten ce ao grupo ortogonal de $Q$ e leva $E$ em $E$ e $E^{*}$ em $E^{*}$.

\section{Prova:}

Verifica-se como em 2.9, que

$$
F^{\prime}: M \rightarrow M \quad t \cdot q \cdot F^{\prime}\left(x, x^{\prime}\right)=\left(u^{\prime}(x), \stackrel{V}{u}^{\prime}\left(x^{\prime}\right)\right)
$$

é um automorfismo linear de $\mathrm{M}$ e

$$
\left\langle u^{\prime}(x), \stackrel{v}{u^{\prime}}\left(x^{\prime}\right)\right\rangle=\left\langle x, x^{\prime}\right\rangle
$$

e que mostra que $F^{\prime}$ pertence ao grupo ortogonal $G$ de $M$.

$F^{\prime}$ leva os s.e. totalmente singulares maximais $E$ e $E^{*}$, neles

mesmos, is to é,

$$
F^{\prime}(E)=E \quad \text { e } \quad F^{\prime}\left(E^{*}\right)=E^{*}
$$

Se $x \in E \subset M$

$$
F^{\prime}(x)=F^{\prime}(x, 0)=\left(u^{\prime}(x), 0\right) \in E
$$

e se $x^{\prime} \in E^{*} \subset M$.

$$
F^{\prime}(x)=F^{\prime}\left(0, x^{\prime}\right)=\left(0, \breve{u}^{\prime}\left(x^{\prime}\right) \in E^{*}\right. \text {. }
$$

Vimos que existe $s, s^{\prime} \in \Gamma$ t.q. $\chi(s) \equiv F$ e $\chi\left(s^{\prime}\right) \equiv F^{\prime}$.

Em particular, se

$$
x \in E, F(x)=u(x)=s x s^{-1}=X(s)(x) \in E^{*}
$$

e para

$$
\mathrm{x}^{\prime} \in \mathrm{E}^{*}, \mathrm{~F}^{\prime}\left(\mathrm{x}^{\prime}\right)=\mathrm{V}\left(\mathrm{x}^{\prime}\right)=\mathrm{s} \mathrm{x}^{\prime} \mathrm{s}^{-1}=\chi(s)(\mathrm{x}) \in \mathrm{E}
$$


Analogamente, se

$$
\begin{aligned}
& x \in E \text { e } x^{\prime} \in E^{*}, \exists s, s^{\prime} \in \Gamma \text { t.q. } \\
& F^{\prime}(x)=u^{\prime}(x)=s x s^{-1} \in E \text { e } \\
& F^{\prime}\left(x^{\prime}\right)=u^{\prime}\left(x^{\prime}\right)=s x^{\prime} s^{-1} \in E^{*} .
\end{aligned}
$$

Observemos que se $s_{1}, s_{2} \in \Gamma$ t.q. $\chi\left(s_{1}\right)(x)=\chi\left(s_{2}\right)(x)$ (nas igualdades 1 e 2), para $x \in E$,

$$
\begin{aligned}
s_{1} \times s_{1}^{-1} & =s_{2} \times s_{2}^{-1} \\
s_{1} \times & =s_{2} \times s_{2}^{-1} s_{1} \\
x & =\left(s_{1}^{-1} s_{2}\right) \times\left(s_{2}^{-1} s_{1}\right) \\
x & =\left(s_{1}^{-1} s_{2}\right) \times\left(s_{1}^{-1} s_{2}\right)^{-1}
\end{aligned}
$$

Portanto,

$$
\begin{aligned}
& \mathrm{s}_{1}^{-1} \mathrm{~s}_{2} \text { pertence ao centro de } \mathrm{C} \Longrightarrow \mathrm{s}_{1}^{-1} \mathrm{~s}_{2} \in \mathrm{K}^{*} \Longrightarrow \\
& \frac{\mathrm{s}_{2}}{\mathrm{~s}_{1}}=\mathrm{k} \in \mathrm{K}^{*} \Longrightarrow \mathrm{s}_{2}=\mathrm{k} \cdot \mathrm{s}_{1}
\end{aligned}
$$

e portanto, $s_{1}$ e $s_{2}$ diferem por um escalar diferente de zero.

Pode-se interpretar ou estudar estas aplicações ortogonais, atra vés das representações.

Definição 2.13. Chama-se representação do grupo $G$ todo homomorfismo $P$ de $G$ no grupo dos automorfismos de um espaço vetorial E, que é o espaço da representação.

Se E é um K-espaço vetorial, de dimensão finita $n$, diz-se que $P$ é uma representação sobre o corpo $k$, de grau $n$. 
Definição 2.14. Sejam P e P' representações de um grupo G e E e E' os espaços destas representações. Diz-se que $P$ e $P^{\prime}$ são equivalentes se exis te um isomorfismo $J$ de $E$ sobre $E^{\prime}$ tal que

$$
P^{\prime}(s) \circ J=J \circ P(s) \quad \text { para todo } s \in G
$$

Definição 2.15. Sejam $P$ e $P^{\prime}$ representações de um grupo G e E e E' os espaços destas representações. Diz-se que $\mathrm{P}$ e $\mathrm{P}^{\prime}$ são contragradientes, se existe uma forma bilinear $B$ não-degenerada sobre $E \times E^{\prime}$ tal que vale

$$
B\left(P(s) x, P^{\prime}(s) x^{\prime}\right)=B\left(x, x^{\prime}\right)
$$

quaisquer que sejam $s \in G, x \in E$ e $x^{\prime} \in E^{\prime}$, diz-se também que $P$ e.P' são contragradientes em relação à forma $B$.

Se $u: E \rightarrow E^{*} \bar{e}$ um isomorfismo linear, determina-se uma forma b $\underline{i}$ linear não-degenerada $B_{1}: E \times E \rightarrow K$ e reciprocamente.

Seja $Q_{1}$ a forma quadrática em E t.q. $Q_{1}(x)=B_{1}(x, x) x \in E \cdot R e-$ presenta-se por $C_{E}$ a älgebra de Clifford de $E$ em relação a forma quadrática $Q_{1}$ e $\Gamma_{E}$ o seu grupo de Clifford.

Se $s \in \Gamma_{E}$, seja $\chi(s)$ o automorfismo linear $x \rightarrow s \times s^{-1}$ de $E$; $\chi \bar{e}$ a representação vetorial de $\Gamma$.

Verifica-se que a aplicação

$$
s . t^{t} x\left(s^{-1}\right)
$$

é uma representação do grupo $\Gamma$, contragradiente à $\chi(s)$ em relação à forma bili near canônica $\left\langle\mathrm{x}, \mathrm{x}^{\prime}>\right.$ sobre $\mathrm{E} \times \mathrm{E}^{*}$. 
De fato:

$$
\begin{aligned}
& B\left(X(s)(x),{ }^{t} \chi\left(s^{-1}\right)\left(x^{\prime}\right)\right)=\left[{ }^{t} \chi\left(s^{-1}\right) x^{\prime}\right](X(s)(x)= \\
& {\left[x^{\prime} \circ X\left(s^{-1}\right)\right](X(s)(x))=x^{\prime}(x)=\left\langle x, x^{\prime}\right\rangle=B\left(x, x^{\prime}\right) x \in E, x^{\prime} \in E^{\prime}}
\end{aligned}
$$

${ }^{t} \chi\left(s^{-1}\right)$ chama-se dual da representação $\chi(s)$ e o espaço desta representação $\bar{e} E^{*}$. A igualdade acima pode ser escrita

$$
\left\langle\chi(s)(x),{ }^{t} \chi\left(s^{-1}\right)\left(x^{\prime}\right)\right\rangle=\left\langle x, x^{\prime}\right\rangle \quad s \in \Gamma
$$

o que mostra que a aplicação

$$
F^{\prime \prime}: M \rightarrow M \quad t \cdot q \cdot F^{\prime \prime}\left(x, x^{\prime}\right)=\left(X(s)(x),{ }^{t} X\left(s^{-1}\right)\left(x^{\prime}\right)\right)
$$

pertence ao grupo ortogonal $G$ de $Q$ e vale $F^{\prime \prime}(E)=E$ e $F^{\prime \prime}\left(E^{*}\right)=E^{*}$, isto $\bar{e}, \quad F^{\prime \prime}$ preserva os s.e. totalmente singulares maximais de $M$.

Vimos que $\mathrm{E}$ e $\mathrm{E}^{*}$ são os espaços das representações $\mathrm{X}(\mathrm{s}) \mathrm{e}^{\mathrm{t}} \chi\left(\mathrm{s}^{-1}\right)$, e considerando-se o isomorfismo u, vale

$$
{ }^{t} \chi\left(s^{-1}\right) \circ u=u \circ \chi(s) \quad \text { para todo } s \in \Gamma_{E}
$$

Resulta de (3) e (5) que $\chi(s)$ e ${ }^{t} \chi\left(s^{-1}\right)$ são representações do grupo $\Gamma_{E}$, que são equivalentes e contragradientes entre si.

De modo geral, se $P$ e $P^{\prime}$ são representações de um grupo $G$, E e $E^{\prime}$ espaços de suas representações (de dim finita) e $B: E \times E^{\prime}$. uma forma bilinear não-degenerada, conclui-se que: se $P^{\prime}$ é contragradiente à $P$, então $P^{\prime} e^{\prime}$ equiva lente à representação dual de $P$.

Resulta que todas as representações contragradientes a uma re presentação de grau finito são equivalentes entre si; além do mais, é claro que, se $\mathrm{P}$ é de grau finito, a dual da dual de $\mathrm{P}$ é equivalente à $\mathrm{P}$. 
Então, para que duas representações de grau finito de um grupo G sejam contragradientes, ē necessārio e suficiente que cada uma seja equivalente à dual da outra.

Observemos finalmente, que a representação $\Lambda=\chi(s) \times{ }^{t} \chi\left(s^{-1}\right)$, produto cartesiano de $\chi(s)$ e ${ }^{t} \chi\left(s^{-1}\right)$, do espaço vetorial $E \times E^{*}$ (que se identifi ca a $F^{\prime \prime}$ ) é contragradiente a ela mesma em relação a $B_{0}$.

$$
B_{0}\left(\left(x+x^{\prime}, y+y^{\prime}\right)\right)=\left[x^{\prime}(y)+y^{\prime}(x)\right] \quad x, y \in E, \quad x^{\prime}, y^{\prime}, \in E^{*}
$$

De fato:

$$
\begin{aligned}
& B_{0}\left(\Lambda(s)\left(x+x^{\prime}\right), \Lambda(s)\left(y+y^{\prime}\right)=\right. \\
& B_{0}\left(\chi(s)(x)+{ }^{t} \chi\left(s^{-1}\right)\left(x^{\prime}\right), X(s)(y)+{ }^{t} \chi\left(s^{-1}\right)\left(y^{\prime}\right)\right)= \\
& { }^{t} \chi\left(s^{-1}\right)\left(x^{\prime}\right)[\chi(s)(y)]+{ }^{t} \chi\left(s^{-1}\right)\left(y^{\prime}\right)[\chi(s)(x)]= \\
& x^{\prime} \circ \chi\left(s^{-1}\right)[X(s)(y)]+y^{\prime} \circ \chi\left(s^{-1}\right)[X(s)(x)]= \\
& x^{\prime}(y)+y^{\prime}(x)=B_{0}\left(x+x^{\prime}, y+y^{\prime}\right) \quad x, y \in E \quad x^{\prime}, y^{\prime} \in E^{*}
\end{aligned}
$$

Teorema 2.16. "Se $\operatorname{dim} M=\mathrm{m}>0$, o grupo $\chi\left(\Gamma^{+}\right) \overline{\mathrm{e}}$ um subgrupo de índice 2 de $G$. Se $K$ não é de característica 2, então $\chi\left(\Gamma^{+}\right)$è o grupo de operações de determinante 1 em G".

\section{Prova}

Desde que $m>0, M$ contém um vetor não singular $x ; x$ é um elemento ímpar de $\Gamma$, donde $\Gamma \neq \Gamma^{+}$.

Se $m$ é par, então o centro de $C_{M}$ é $K .1$, que está em $C_{+}$; segue imediatamente que $\chi\left(\Gamma^{+}\right)$é então de indice $2 \mathrm{em} \mathrm{G}$. 
Se mé impar, então o centro de $C_{M}$ contēm um elemento inversível ímpar; este élemento está em $\Gamma$ mas não em $\Gamma_{+}$.

Então segue de [2.5] que todo elemento de $\Gamma$ é o produto de um elemento do centro de $C_{M}$ por um elemento de $\Gamma^{+}$, donde $\chi(\Gamma)=\chi\left(\Gamma^{+}\right)$. Este grupo é - grupo de operações de determinante 1 em G.

0 determinante de todo elemento de $G \bar{e} \pm 1$, e $G$ contém uma operação de determinante -1 , por exemplo, a aplicação $x \rightarrow-x$. Segue $\chi\left(\Gamma^{+}\right) e \overline{\text { de }}$ índi ce 2 em G.

Agora, suponhamos que $\underline{\mathrm{m}} \overline{\mathrm{e}} \mathrm{par}$ e que a característica de $\mathrm{K} \ddot{\mathrm{e}} \neq 2$. Todo $s \in \Gamma$ é representável na forma $c_{1} \ldots x_{h}, c \in k, x_{i} \in \Gamma \cap M(1 \leq i \leq h)$ e vê-se facilmente que det $x\left(x_{i}\right)=-1 \quad(1 \leq i \leq h)$, donde

$$
\operatorname{det} \chi(s)=(-1)^{h} \text {. }
$$

Então, det $\chi(s)$ é 1 ou -1 , se's $\in \Gamma^{+}$ou não, o que completa a prova do teorema.

Ogrupo $\Gamma^{+}$chama-se grupo especial de Clifford de Q; 0 grupo $\chi\left(\Gamma^{+}\right)$será chamado grupo especial ortogonal de $Q$ (ou grupo de rotações).

Teorema 2.17. [8] Se m é par $>0$, o grupo $\Gamma^{+}$é gerado pelos produtos de dois elementos não singulares de $M$.

Conclui-se que:

(A) As operações $F_{i}$ de $G$ que trocam os s.e. totalmente singulares maximais $E$ e $E^{*}$ de M são "equivalentes" à aplicação F.

$F_{i} \equiv F(\bmod u) u$ isomorfismo de $E$ em $E^{*}$ e se $s \in \Gamma$ t.q. $\chi(s) \equiv F_{i}$, então

$$
\operatorname{det} x(s)=-1
$$


(B) As operações $F$ ! de $G$ que conservam os s.e. totalmente singulares maximais $E$ e $E^{*}$ de $M$ são "equivalentes" à aplicação $F^{\prime}$.

$F_{j}^{\prime} \equiv F^{\prime}\left(\bmod u^{\prime}\right) u^{\prime}$ isomorfismo de $E$ em $E$ e se $s \in \Gamma$ t.q. $\chi(s) \equiv F_{j}^{\prime}$ então

$$
\operatorname{det} x(s)=1
$$

Obtemos então uma partição do grupo G que corresponde a uma par tição do grupo de Clifford $\Gamma_{M}$, que originam respectivamente as reflexões e rotações de M.

$$
\begin{aligned}
& G=\left\{F_{i} \in G \text { t.q. } F_{i}(E)=E^{*} \text { e } F_{i}\left(E^{*}\right)=E\right\} U\left\{F_{j}^{\prime} \in G t \cdot q \cdot F_{j}^{\prime}(E)=E \text { e } F_{j}^{\prime}\left(E^{*}\right)=E^{*}\right\} \\
& \Gamma=\{s \text { t.q. det } \chi(s)=-1\} \cup\{s \text { t.q. } \operatorname{det} \chi(s)=1\}
\end{aligned}
$$

Podemos verificar esta afirmação do seguinte modo:

Sabemos que as matrizes quadradas inversíveis sobre $\mathrm{K}$ (de ordem

n) correspondem aos automorfismos $u^{\prime}$ de $E$. Se $A=M\left(u^{\prime}\right)$ e a matriz de $u^{\prime}$ em relação à base $\left(x_{i}\right)_{1 \leq i \leq n}, M(\stackrel{v}{u})=\left({ }^{t} A\right)^{-1}={ }^{t}\left(A^{-1}\right)$, que é a matriz contragradiente de $X$.

Então, a matriz de $F^{\prime}$ em relação a uma base $\left(x_{1} \ldots, x_{n}, y_{1}^{\prime}, \ldots, y_{n}^{\prime}\right)$ de M é dada por:

$$
M\left(F^{\prime}\right)=\left[\begin{array}{cc}
A & 0 \\
0 & t_{A}^{-1}
\end{array}\right]_{2 n \times 2 n}
$$

$e \operatorname{det} M\left(F^{\prime}\right)=\operatorname{det} A \cdot \operatorname{det}{ }^{t} A^{-1}=\operatorname{det} A \cdot \operatorname{det} A^{-1}=1$. 
Analogamente, se $\mathrm{B}=M(\mathrm{u})$ é a matriz de $\mathrm{u}: \mathrm{E} \rightarrow \mathrm{E}^{*}$, em relação às bases $\left(x_{i}\right)_{1 \leq i \leq n}$ de $E$ e $\left(y_{j}^{\prime}\right)_{1 \leq j \leq n}$ de $E^{*}$, então $M(\tilde{u})=\left({ }^{t} B\right)^{-1}={ }^{t}{ }^{-1}$.

A matriz de $F$ em relação à base $\left(x_{1}, \ldots, x_{n}, y_{1}^{\prime}, \ldots, y_{n}^{\prime}\right)$ de $M \bar{e}$ da da por:

$$
M(F)=\left[\begin{array}{cc}
0 & B \\
t_{B}-1 & 0
\end{array}\right]
$$

$\mathrm{e} \operatorname{det} M(F)= \pm 1$. (se n é par ou ímpar).

Vimos que para $F \in G$, existe $s \in \Gamma\left(\Gamma_{M}\right)$ tal que $X(s) \equiv F$.

Se

$$
\begin{aligned}
z & =\left(x, x^{\prime}\right) \in M, \quad s z s^{-1}=F(z) \Rightarrow s\left(x, x^{\prime}\right) s^{-1}= \\
& =\left(u(x), u\left(x^{\prime}\right)\right)
\end{aligned}
$$

e se $z=x$ ou $z=x^{\prime}$, temos:

$$
s \times s^{-1}=u(x) \quad \text { e } \quad s x^{\prime} s^{-1}=\stackrel{v}{u}\left(x^{\prime}\right) \quad s \in \Gamma_{M}
$$

$u: E \rightarrow E^{*}$, o isomorfismo linear jā definido.

$$
\begin{aligned}
& \text { Se } u(x)=x(s)(x) \\
& \quad u^{-1}(x)=[x(s)]^{-1}(x)=\chi\left(s^{-1}\right)(x)
\end{aligned}
$$

e

$$
t_{u}^{-1}={ }^{t} \times\left(s^{-1}\right) \quad s \in \Gamma_{M}
$$

Então

$$
\left.F\left(x, x^{\prime}\right)=(u(x), \stackrel{v}{u})\left(x^{\prime}\right)\right)=\left(x(s)(x),{ }^{t} \chi\left(s^{-1}\right)\left(x^{\prime}\right)\right) \quad x \in E, \quad x^{\prime} \in E^{*}
$$


- que implica que

$$
\begin{gathered}
\chi(s) \equiv \mathrm{u} \quad: \mathrm{E} \rightarrow \mathrm{E}^{*} \\
\mathrm{t}_{\chi\left(\mathrm{s}^{-1}\right) \equiv \mathrm{u}}: \mathrm{E}^{*} \rightarrow \mathrm{E}
\end{gathered}
$$

são representações do grupo $\Gamma_{M}$ sobre o espaço vetorial $M$.

Analogamente, se u é um automorfismo linear de $E$, e $F^{\prime}: M \rightarrow M \quad o$ automorfismo definido anteriormente, vale:

$$
\equiv s \in \Gamma_{M} \text { t.q. } \chi(s) \equiv F^{\prime}
$$

Se

$$
\begin{aligned}
& z=\left(x, x^{\prime}\right) \in M ; X(s)(z)=F^{\prime}(z) \Longrightarrow s^{-1}=F^{\prime}(z) ; \\
& s\left(x, x^{\prime}\right) s^{-1}=\left(u(x), \stackrel{v}{u}\left(x^{\prime}\right)\right)
\end{aligned}
$$

e se $z=x$ ou $z=x^{\prime}$, fica:

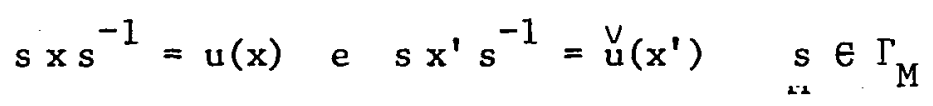

Neste caso, $X(s)=u: E \rightarrow E$ e ${ }^{t} X\left(s^{-1}\right)=\mathrm{U}_{\mathrm{U}} \mathrm{E}^{*} \rightarrow \mathrm{E}^{*}$ são representação do grupo $\Gamma_{M}$ e pode-se verificar que estas aplicações podem ser consideradas como representações do grupo $\Gamma_{E}$ (e os espaços das representações são $E$ e $E^{*}$ ). Aplicando-se o texto de Bourbaki [4] sobre aplicações lineares de uma soma direta, temos:

Proposição 2.18. Seja $\mathrm{M}=\mathrm{E} \oplus \mathrm{E}^{*}$ e $\mathrm{N}$ um $\mathrm{K}$-espaço vetorial; qualquer que sejam as aplicações lineares $\mathrm{F}_{\mathrm{E}}: \mathrm{E} \rightarrow \mathrm{N}$ e $\mathrm{F}_{\mathrm{E}^{*}}: \mathrm{E}^{*} \rightarrow \mathrm{N}$, existe uma aplicação linear $F$ e uma só de $M$ em $N$ t.q. a restrição de $F$ à $E$ (ou à $E^{*}$ ) é igual à $F_{E}$ (respectivamente $F_{E^{*}}$ ).

Em particular, se $F: M \rightarrow M e ́$ dada por $F\left(x, x^{\prime}\right)=\left(u(x), \quad u^{\prime}\left(x^{\prime}\right)\right)$, te mos: 


$$
\begin{aligned}
F(z) & =F\left(h_{E}(z)+h_{E^{*}}(z)\right) \quad z \in M \\
& =F\left(h_{E}(z)\right)+F\left(h_{E^{*}}(z)\right)= \\
& =F_{E}\left(h_{E}(z)\right)+F_{E^{*}}\left(h_{E} *(z)\right) \\
& =F_{E}(x)+F_{E^{*}}\left(x^{\prime}\right)=u(x)+v\left(x^{\prime}\right)
\end{aligned}
$$

onde $\mathrm{F}_{\mathrm{E}}=\mathrm{u}: \mathrm{E} \rightarrow \mathrm{E}^{*}$ e $\mathrm{F}_{\mathrm{E}} *=\stackrel{\mathrm{V}}{\mathrm{u}}: \mathrm{E}^{*} \rightarrow \mathrm{E}$ e $\mathrm{h}_{\mathrm{E}}(\mathrm{z})$ (respectivamente $\mathrm{h}_{\mathrm{E}} *(\mathrm{z})$ representa a componente de $z$ em $E\left(e m E^{*}\right)$.

$$
\begin{aligned}
& \text { Analogamente, vale para } F_{E}^{\prime}=u: E \rightarrow E \text { e } F_{E^{*}}^{\prime}=\mathrm{v}: E^{*} \rightarrow E^{*} \\
& O \text { valor de } F \text { para todo } z \text { de } M \text { é determinado pelo conhecimento }
\end{aligned}
$$
das restrições de $\mathrm{F}$ a $\mathrm{E}$ ( $\mathrm{e}$ à $\left.\mathrm{E}^{*}\right)$.

Reciprocamente, conhecendo-se as aplicações lineares $F_{E}=u: E \rightarrow M$ e $\mathrm{F}_{\mathrm{E}^{*}}=\mathrm{\textrm {U }}: \mathrm{E}^{*} \rightarrow \mathrm{M}$, determina-se uma única aplicação $\mathrm{F}: \mathrm{M} \rightarrow \mathrm{M}$.

Por outro lado, se considerarmos as aplicações lineares

$$
\begin{aligned}
& u_{1}: E \rightarrow E \oplus E^{*} \\
& u_{2}: E^{*} \rightarrow E \oplus E^{*}
\end{aligned}
$$

e se $z \in E \oplus E^{*}$, representa-se $k_{1}(z)$ a componente de $z$ em $E \cdot e k_{2}(z)$ a componente de $z$ em $E^{*}$.

$$
\begin{aligned}
& \text { Dada } F: M \rightarrow E \oplus E^{*}, F=u_{1}+u_{2} \text {, vale: } \\
& u_{1}=k_{1} \circ u_{1}+k_{2} \circ u_{1} \text { e } u_{2}=k_{1} \circ u_{2}+k_{2} \circ u_{2}
\end{aligned}
$$

e então:

$$
F(z)=k_{1} \circ u_{1}(z)+k_{2} \circ u_{1}(z)+k_{1} \circ u_{2}(z)+k_{2} \circ u_{2}(z)
$$

onde as aplicações lineares são definidas

$$
\begin{aligned}
& \mathrm{k}_{1} \circ \mathrm{u}_{1}: E \rightarrow E \\
& \mathrm{k}_{2} \circ \mathrm{u}_{1}: E \rightarrow E^{*} \\
& \mathrm{k}_{1} \circ \mathrm{u}_{2}: \mathrm{E}^{*} \rightarrow \mathrm{E} \\
& \mathrm{k}_{2} \circ \mathrm{u}_{2}: \mathrm{E}^{*} \rightarrow \mathrm{E}^{*}
\end{aligned}
$$

Verifica-se que as aplicações lineares definidas acimas (t.q. $\mathrm{u}_{1}=\mathrm{u}$ e $\mathrm{u}_{2}=\stackrel{\mathrm{v}}{\mathrm{u}}$ ) geram todas as operações do grupo $\mathrm{G}$. 
$\mathrm{E}$ e $\mathrm{E}^{*}$ não são os únicos subespaços totalmente singulares maximais de M. De fato:

Seja $S_{1}$ um subespaço regular de $E$ e seja $S_{1}^{\downarrow}$ o subespaço de $E^{*}$ ortogonal à $\mathrm{S}_{1}$ em relação à forma bilinear canônica, isto é,

$$
S_{1}^{\perp}=\left\{y^{\prime} \in E^{*} /\left\langle x, y^{\prime}\right\rangle=0 \quad \forall x \in S_{1}\right\}
$$

Deduz-se que: todo subespaço $S_{1}$ regular de $E \bar{e}$ um fator direto e $E=S_{1} \oplus S_{1}^{\perp}$, e que $\operatorname{dim}\left(S_{1} \oplus S_{1}^{\perp}\right)=\operatorname{dim} E=n$.

Conclui-se então que o subespaço $S_{1} \oplus S_{1}^{\perp}$ de $M \bar{e}$ totalmente singular maximal, em relação à forma quadrática canônica.

Proposição 1: Seja $\mathrm{E}=\mathrm{S}_{1} \oplus \mathrm{S}_{2}$, uma decomposição de $\mathrm{E}$ como soma direta de subespaços. Então

$$
\mathrm{E}^{*}=\mathrm{S}_{1}^{1} \oplus \mathrm{S}_{2}^{\perp}
$$

e os pares $\mathrm{S}_{1}^{\perp}, \mathrm{S}_{2}$ e $\mathrm{S}_{2}^{\perp}, \mathrm{s}_{1}$ são duais em relação à forma bilinear canônica. Além disso, as aplicações injetoras induzidas

$$
s_{1}^{\perp} \rightarrow s_{2}^{*}, \quad s_{2}^{\perp} \rightarrow s_{1}^{*}
$$

são sobrejetoras e portanto,

$$
\mathrm{E}^{*}=\mathrm{S}_{1}^{*} \oplus \mathrm{S}_{2}^{*}
$$

Prova: Sejam $\pi_{1}: E \rightarrow E_{1}$ e $\pi_{2}: E \rightarrow E_{2}$ as projeções canônicas associadas com a soma direta.

$$
\begin{aligned}
& \text { Seja } x^{\prime} \in E^{*} \text {, e define-se } x_{1}^{\prime}, x_{2}^{\prime} \text { por } \\
& \qquad x_{1}^{\prime}(x)=x^{\prime}\left(\pi_{2} x\right) \text { e } x_{2}^{\prime}(x)=x^{\prime}\left(\pi_{1} x\right)
\end{aligned}
$$


Segue que $x_{i}^{\prime} \in S_{i}^{1}(i=1,2)$ e $x^{\prime}=x_{1}^{\prime}+x_{2}^{\prime}$. Portanto,

$$
\mathrm{E}^{*}=\mathrm{S}_{1}^{\perp}+\mathrm{S}_{2}^{1}
$$

Para mostrar que a decomposição é direta, suponhamos que

$$
\begin{array}{ll}
x^{\prime} \in s_{1}^{\perp} \cap s_{2}^{\perp} . & \text { Então } \\
x^{\prime}(x)=0 & x \in s_{1}, x \in s_{2}
\end{array}
$$

e portanto $x^{\prime}(x)=0$ para todo $x \in E$. Então $x^{\prime}=0$ e assin a decomposição é direta.

Proposição 2 - Seja $S_{1} \subset E$ um subespaço e seja $S_{1}^{*} \subset E^{*}$ o subespaço dual de $E_{1}$ tal que

$$
\left(s_{1}^{*}\right)^{\perp 1}=s_{1}^{*}
$$

Então

$$
E=S_{1} \oplus\left(S_{1}^{*}\right)^{\perp}
$$

e

$$
E^{*}=S_{1}^{*} \oplus S_{1}^{\perp}
$$

Prova: Temos

$$
\left(s_{1}+s_{1}^{*}\right)^{\perp}=s_{1}^{\perp} n\left(s_{1}^{*}\right)^{\perp}=s_{1}^{\perp} n s_{1}^{*}=0
$$

donde

$$
E=0^{\perp}=\left[S_{1}+\left(s_{1}^{*}\right)\right]^{\perp \perp}=S_{1}+S_{1}^{* \perp}
$$


Por outro lado, desde que $S_{1}$ e $S_{1}^{*}$ são duais, segue que

$$
s_{1} \cap s_{1}^{*}=0
$$

- que junto com (3) prova que a soma é direta.

(2) segue da Proposição anterior.

Dado o isomorfismo linear $\mathrm{u}: \mathrm{E} \rightarrow \mathrm{E}^{*}$ e $\stackrel{\mathrm{v}}{\mathrm{u}: \mathrm{E}^{*}} \rightarrow \mathrm{E}$, podemos escrever:

$$
\begin{aligned}
& u_{\mid s_{1}}=u_{1} \text { e }\left.{ }^{u}\right|_{S_{2}}=u_{2} \\
& \stackrel{v}{u}_{\mid S_{2}^{\perp}}=\stackrel{v}{u}_{1} \text { e }{ }^{\stackrel{v}{u}} \mid s_{1}^{1}=\stackrel{v}{u}_{2}
\end{aligned}
$$

Então temos:

$$
E=S_{1} \oplus S_{2} \stackrel{\left(I, u_{2}\right)}{\longrightarrow} s_{1} \oplus S_{1}^{\perp} \stackrel{\left(u_{1}, I\right)}{\longrightarrow} s_{2}^{\perp} \oplus S_{1}^{\perp} \cong S_{1}^{*} \oplus S_{2}^{*}=E^{*}
$$

Reciprocamente, dadas as aplicações:

$$
\begin{aligned}
& u_{1}: s_{1} \longrightarrow s_{2}^{\perp} \\
& u_{2}: s_{2} \longrightarrow s_{1}^{\perp}
\end{aligned}
$$

que caracterizam a dualidade, obtém-se uma aplicação linear

$$
\begin{gathered}
\mathrm{u}: \mathrm{E} \rightarrow \mathrm{E}^{*} . \\
\text { Analogamente, temos: } \\
\mathrm{E}^{*}=\mathrm{S}_{1}^{*} \oplus \mathrm{S}_{2}^{*} \cong \mathrm{S}_{2}^{\perp} \oplus \mathrm{S}_{1}^{\perp} \stackrel{\left(\mathrm{u}_{1}, I\right)}{\longrightarrow} \mathrm{S}_{1} \oplus \mathrm{S}_{1}^{\perp} \stackrel{\left(\mathrm{I}, \mathrm{u}_{2}\right)}{\longrightarrow} \mathrm{S}_{1} \oplus \mathrm{S}_{2}=\mathrm{E}
\end{gathered}
$$


e obtém-se os isomorfismos que permutam entre si"os subespaços totalmente singulares maximais de M.

Além disso, observemos que:

$$
\begin{aligned}
& E \stackrel{u}{\longrightarrow} E^{*} \stackrel{\stackrel{v}{\longrightarrow}}{\longrightarrow} E \stackrel{v}{u} \circ u: E \rightarrow E \\
&(\left.=I_{E} \text { se u é simétrica }\right) \\
& E^{*} \stackrel{\stackrel{v}{u}}{\longrightarrow} E \stackrel{\text { u }}{\longrightarrow} E^{*} \quad \text { u } \circ \stackrel{v}{u}: E^{*} \rightarrow E^{*} \\
&\left(=I_{E}^{*} \text { se u é simétrica }\right)
\end{aligned}
$$

Sabemos que $\mathrm{S}_{1}^{\perp}$ e $\mathrm{S}_{2} ; \mathrm{S}_{2}^{\perp}$ e $\mathrm{S}_{1}$ são duais em relação à forma bilinear canônica e o subespaço $S_{1} \oplus S_{1}^{\perp}$ de $M$ é totalmente singular maximal em relação à forma quadrätica $Q\left(Q\left(x, y^{\prime}\right)=\left\langle x, y^{\prime}\right\rangle\right)$, considerando $\operatorname{dim}\left(S_{1} \oplus s_{1}^{\perp}\right)=n$.

Portanto, dado qualquer subespaço $S_{i}$ regular de $M$, obtém-se as decomposições acima descritas e as aplicações lineares que permutam entre si os subespaços totalmente singulares maximais de $M$.

Em particular, temos o caso em que

$$
\mathrm{S}_{1}=\mathrm{E} \quad \text { e } \quad \mathrm{s}_{1}^{\perp}=\{0\}
$$

As operações do grupo $G$, isto é, os automorfismos de $M$ que conservam a forma quadrática, são formados por pares de aplicações que permutam entre si os subespaços totalmente singulares maximais de $\mathrm{M}$, por exemplo:

$$
\begin{aligned}
& \mathrm{F}_{1}=(\mathrm{u}, \mathrm{v}) \quad \mathrm{u}: \mathrm{E} \rightarrow \mathrm{E}^{*} \text { isomorfismo linear }
\end{aligned}
$$

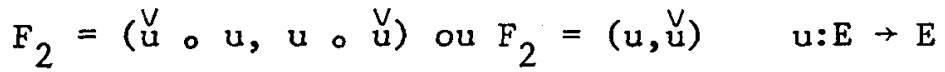

e analogamente, determinam-se todos os automorfismos $F_{i} \in G$.

Conclui-se que os isomorfismos lineares acima geram o grupo G. 


\section{CAPÍTULO III}

\section{RELAॄÕES ENTRE AS ÁLGEBRAS DE CLIFFORD E ÁLGEBRA EXTERIOR}

Vimos a definição e construção da álgebra de clifford que: dado $z \in M$, a aplicação $\phi: z \rightarrow L_{z}^{\prime}$ de $M$ em End $(\Lambda M)=\mathbb{C}$ (endomorfismos de espaços vetorial $\Lambda$ M), satisfaz a relação

$$
L_{z}^{2}=Q(z) \cdot I \quad z \in M
$$

Isto implica que existe um homomorfismo $\psi: C \rightarrow C$ que estende $z \rightarrow L_{z}^{\prime} \bar{e}$ um isomorfismo de $M+$ End $(\Lambda M)$.

Dada uma forma bilinear $B_{0}: M \times M \rightarrow K$ tal que $Q(z)=B_{0}(z, z)$ para $z \in M$, podemos identificar o espaço vetorial subjacente da álgebra de Clifford $C_{M}$ com o espaço vetorial básico da álgebra exterior $\Lambda M$ de $M$, tal que

$$
z \cdot w=\left(L_{z}+\delta_{z}\right)(w)=z \wedge w+\delta_{z} \cdot w \quad z, w \in M
$$

onde $\delta_{z}: \Lambda M \rightarrow \Lambda M$ é a anti-derivação tal que $\delta_{z} w=B_{0}(z, w) \cdot 1$.

[Observemos que se $z$, w $\in M, z \cdot$ w representa seu produto em $C_{M}$, enquanto $z \wedge$ w representa seu produto em $\Lambda \mathrm{M}]$.

Pode-se escrever então

$$
z \cdot w=z \wedge w+B_{0}(z, w) \quad z, w \in M
$$

onde $B_{o} \bar{e}$ a forma bilinear associada à $Q$.

$$
\text { No caso em questão, no qual } M=E \oplus E^{*} \text { e } Q(z)=\left\langle x, x^{\prime}\right\rangle \dot{e} \text { a for- }
$$

ma quadrática canônica, a relação (3) se escreve:

Se

$$
z=x+x^{\prime}, \quad w=y+y^{\prime} \quad \in M
$$




$$
\begin{aligned}
&\left(x+x^{\prime}\right)\left(y+y^{\prime}\right)=\left(x+x^{\prime}\right) \wedge\left(y+y^{\prime}\right)+\frac{1}{2}\left[x^{\prime}(y)+y^{\prime}(x)\right] \cdot 1 \\
& x \cdot y+x y^{\prime}+x^{\prime} y+x^{\prime} \cdot y^{\prime}= x \wedge y+x \wedge y^{\prime}+x^{\prime} \wedge y+x^{\prime} \wedge y^{\prime}+ \\
&+\frac{1}{2}\left[x^{\prime}(y)+y^{\prime}(x)\right] \cdot 1
\end{aligned}
$$

mas:

$$
x \cdot y=x \wedge y \quad \text { e } x^{\prime} \cdot y^{\prime}=x^{\prime} \wedge y^{\prime} \text { pois: } C_{(E)}=\Lambda E \text { e } C_{\left(E^{*}\right)}=\Lambda E^{*}
$$

Então (4) fica:

$$
x y^{\prime}+x^{\prime} y=x \wedge y^{\prime}+x^{\prime} \wedge y+\frac{1}{2}\left[x^{\prime}(y)+y^{\prime}(x)\right] \cdot 1
$$

se $\quad x, y \in E, \quad x^{\prime}, y^{\prime} \in E^{*}$

Se fizermos em (5), respectivamente $z=x+0^{\prime}$ e $z=0+x^{\prime}$ temos as igualdades;

$$
\begin{aligned}
x y^{\prime} & =x \wedge y^{\prime}+B\left(\left(x, y+y^{\prime}\right)\right) \\
& =x \wedge y^{\prime}+\left\langle x, y^{\prime}\right\rangle \\
x^{\prime} y & =x^{\prime} \wedge y+B\left(\left(x^{\prime}, y+y^{\prime}\right)\right) \\
& =x^{\prime} \wedge y+\left\langle y, x^{\prime}\right\rangle
\end{aligned}
$$

o que pode ser obtido, tomando-se a forma $B=2 B_{0}$.

Se tomarmos a base $\left(x_{i}\right)_{1 \leq i \leq n}$ de $E$ e a base dual $\left(y_{j}^{\prime}\right)_{1 \leq j \leq n}$ de $E^{*}$, temos

$$
\begin{aligned}
& x_{i} \cdot y_{j}^{\prime}=x_{i} \wedge y_{j}^{\prime}+\delta_{i j} \cdot 1 \\
& y_{j}^{\prime} \cdot x_{i}=y_{j}^{\prime} \wedge x_{i}+\delta_{j i} \cdot 1
\end{aligned}
$$

Além disso, é importante observar que (6.a) e (6.b) implicam Se

$$
z_{1}=x_{1}+y_{1}^{\prime}, z_{2}=x_{2}+y_{2}^{\prime} \quad \in M
$$


então

$$
\begin{aligned}
& x_{1} y_{2}^{\prime}=x_{1} \wedge y_{2}^{\prime}+B_{1}\left(x_{1}, y_{2}\right) \\
& y_{1}^{\prime} x_{2}=y_{1}^{\prime} \wedge x_{2}+B_{1}\left(x_{2}, y_{1}\right)
\end{aligned}
$$

onde $B_{1}: E \times E \rightarrow K$ é a forma bilinear simétrica t.q. $\sigma(y)(x)=B_{1}(x, y)$ e $\sigma=u: E \rightarrow E$ * é o isomorfismo linear do capítulo anterior ou $B_{1}(x, y)=\frac{1}{2}[\sigma(y)(x)+\sigma(x)(y)]$.

Retomamos a construção de uma álgebra de clifford, como feita por Chevalley [8].

Queremos definir uma álgebra de Clifford do K-espaço vetorial E, em relação à uma forma quadrática $Q$, (associada à forma bilinear $B_{1}$ ) e estabelecer relações entre esta ālgebra e a álgebra $C$, do K-espaço vetorial M em relação a forma quadrática canônica $Q$, definida anteriormente. Representaremos esta álge bra por $C_{M}$, e fazemos aqui a restrição que a característica do corpo $\mathrm{K}$ deve ser diferente de 2 .

Seja $x^{\prime}$ uma forma linear sobre E. Então existe uma anti-deriva ção $\delta_{x^{\prime}}$ de $\Lambda E$ tal que $\delta_{x^{\prime}}(x)=x^{\prime}(x) \cdot 1$ para todo $x \in E$.

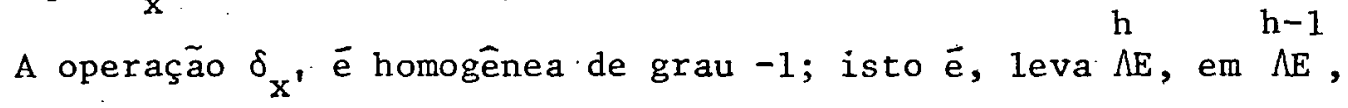
e 1 sobre $\{0\}$. Temos $\delta_{x^{\prime}}^{2}=0$; se $x_{1}^{\prime}, x_{2}^{\prime}$ são formas lineares e $a_{1}, a_{2}$ escalares, então temos

$$
\begin{aligned}
& \delta_{a x^{\prime}}=a \cdot \delta_{x^{\prime}}, \delta_{x_{1}^{\prime}}+x_{2}^{\prime}=\delta_{x_{1}^{\prime}}+\delta_{x_{2}^{\prime}} \\
& \delta_{x_{1}^{\prime}} \delta_{x_{2}^{\prime}}+\delta_{x_{2}^{\prime}} \delta_{x_{1}^{\prime}}=0
\end{aligned}
$$

Se $z=x+\sigma(x)$ é um elemento de $M_{1} \subset$ M definimos a aplica ção linear $\phi_{1}: M_{1} \rightarrow$ End $(\Lambda E)$ da seguinte maneira:

$$
\begin{aligned}
L_{1 z}^{\prime}(y) & =L_{x} \cdot y+\delta_{x^{\prime}}(y)=x \wedge y+x^{\prime}(y) \cdot 1 \\
& =x \wedge y+B_{1}(x, y) \cdot 1
\end{aligned}
$$


onde $B_{1}(x, y)=\sigma(x)(y)=x^{\prime}(y)$ è uma forma bilinear simétrica e $\sigma: E \rightarrow E^{*} \bar{e}$ a apli cação linear definida anteriormente. Então temos:

$$
\begin{aligned}
\delta_{x}^{\prime} L_{x} \cdot y=\delta_{x^{\prime}}(x \wedge y) & =\left(\delta_{x^{\prime}}(x)\right) \wedge y-x \wedge\left(\delta_{x^{\prime}}(y)\right)= \\
& =Q(z) \cdot y-L_{x} \delta_{x^{\prime}}(y)
\end{aligned}
$$

Portanto:

$$
L_{x} \delta_{x^{\prime}}+\delta_{x^{\prime}} L_{x}=Q(z) \cdot I_{E} \quad z \in M_{1}
$$

Desde que $\mathrm{L}_{\mathrm{x}}^{2}=0,(10)$ implica que

$$
L_{1 z}^{\prime 2}=Q(z) \cdot I_{E} \quad z \in M_{1}
$$

Aplicando-se o Teorema 1.16 , a igualdade (11) implica que existe um homomorfismo $\psi_{1}$ de $\mathrm{C}_{\mathrm{M}_{1}}$ em End $(\Lambda E)$ que estende $\phi_{1}$.

os resultados acima são reunidos no

Teorema 3.1. "Seja $\phi_{1}$ a aplicação linear de $M_{1}$ na älgebra dos endomorfismos de $\Lambda E$ definida por (9). Então $L_{1 z}^{\prime 2}=Q(z) \cdot I_{E}$ para $z \in M_{1}$ e $\phi_{1}$ po de ser estendido a um homomorfismo $\psi_{1}$ de $\mathrm{C}_{\mathrm{M}_{1}}$ em End $(\Lambda E)$ ".

Usando a forma bilinear $B_{1}: E \times E \rightarrow K$, define-se uma forma qua drática $Q_{1}$ tal que $Q_{1}(x)=B_{1}(x, x)$ para todo $x \in E$ e a álgebra de Clifford de $E$ em relação à forma quadrätica $Q_{1}$, que será representada por $C_{E}$.

Analogamente, podemos definir o endomorfismo de $\Lambda \mathrm{E}^{*}$ :

$$
L_{2 z}^{\prime}\left(y^{\prime}\right)=L_{x} y^{\prime}+\delta_{x}\left(y^{\prime}\right)=x^{\prime} \wedge y^{\prime}+y^{\prime}(x) \cdot 1
$$

se $z \in M_{2}, y^{\prime} \in E^{*}$, onde $L_{x^{\prime}} \bar{e}^{-}$o operador $y^{\prime} \rightarrow x^{\prime} \wedge y^{\prime}$ da multiplicação por $x^{\prime}$ en $\Lambda E^{*}$ e $\delta_{x}$ a anti-derivação de $\Lambda E^{*}$ tal que

$$
\delta_{x} \cdot y^{\prime}=y^{\prime}(x) \quad y^{\prime} \in E^{*}
$$


e $\quad M_{2}=\left\{z \in M t \cdot q \cdot z=\left(\sigma^{-1}\left(y^{\prime}\right), y^{\prime}\right), y^{\prime} \in E^{*}\right\}$

$$
\mathrm{L}_{2 z}\left(y^{\prime}\right)=x^{\prime} \wedge y^{\prime}+B_{2}\left(x^{\prime}, y^{\prime}\right) \cdot I \text { para } x^{\prime}, y^{\prime} \in E^{*} \text { onde } B_{2}: E^{*} \times E^{*}+K
$$

$\bar{e}$ uma forma bilinear simétrica tal que $B_{2}\left(x^{\prime}, y^{\prime}\right)=y^{\prime}(x)$ e $x=\sigma^{-1}\left(x^{\prime}\right)$

$\sigma^{-1}: E^{*}+E$ é a inversa da aplicação linear definida anteriormente.

Então temos:

$$
\begin{aligned}
& \delta_{x} \cdot L_{x^{\prime}} \cdot y^{\prime}=\delta_{x}\left(x^{\prime} \wedge y^{\prime}\right)= \\
= & \left(\delta_{x^{\prime}}\right) \wedge y^{\prime}-x^{\prime} \wedge \delta_{x}\left(y^{\prime}\right)= \\
= & x^{\prime}(x) \wedge y^{\prime}-L_{x} \delta_{x}\left(y^{\prime}\right)= \\
= & Q(z) \cdot y^{\prime}-L_{x^{\prime}} \delta_{x}\left(y^{\prime}\right)
\end{aligned}
$$

Portanto:

$$
\mathrm{L}_{\mathrm{x}^{\prime}} \delta_{\mathrm{x}}+\delta_{\mathrm{x}} \mathrm{L}_{\mathrm{x}^{\prime}}=\mathrm{Q}(\mathrm{z}) \cdot \mathrm{I}_{\mathrm{E}}{ }^{*}
$$

Desde que $L_{x^{\prime}}^{2}=0,(13)$ implica que

$$
L_{2 z}^{\prime 2}=Q(z) \cdot I_{E} \quad \cdot z \in M_{2}
$$

Temos então uma aplicação linear $\phi_{2}: E^{*}+$ End $\left(\Lambda E^{*}\right)$, aplicando-se o Teorema [1.16], a igualdade (14) implica na existência de um homomorfismo $\psi_{2}$ de $C_{E}{ }^{*}$ em End $\left(\Lambda E^{*}\right)$ que estende $\phi_{2}$.

Temos o seguinte resultado:

Teorema 3.2. "Seja $\phi_{2}$ a aplicação linear de $M_{2}$ na álgebra dos endomorfismos de $\Lambda E^{*}$ definida por (12). Então $L_{2 z}^{\prime 2}=Q(z) \cdot I_{E}{ }^{*}$ para $z \in M_{2} e$ $\phi_{2}$ pode ser estendida a um homomorfismo $\psi_{2}$ de $C_{M_{2}}$ em End $\left(\Lambda E^{*}\right)$." 
Usando a forma bilinear $\mathrm{B}_{2}: \mathrm{E}^{*} \times \mathrm{E}^{*} \rightarrow \mathrm{K}$ define-se uma forma quadrática $Q_{2}$ tal que $Q_{2}\left(x^{\prime}\right)=B_{2}\left(x^{\prime}, x^{\prime}\right)$ para todo $x^{\prime} e E^{*}$ e temos a álgebra de Clifford de $E^{*}$ em relação à forma quadrätica $Q_{2}$, que serā representada por $C_{E}{ }^{*}$.

Definição 3.3. Se $E_{1}, E_{2}$ são espaços vetoriais munidos, respectivamente, de for mas quadräticas $Q_{1}$ e $Q_{2}$ associadas às formas bilineares $B_{1}$ e $B_{2}$, dizemos que eles são isométricos se existe um isomorfismo linear $\tau: E_{1} \rightarrow E_{2}$ tal que

$$
B_{2}(\tau(x), \tau(y))=B_{1}(x, y) \text { para todo } x, y \in E_{1}
$$

Notação: $\quad\left(E_{1}, Q_{1}\right) \simeq\left(E_{2}, Q_{2}\right)$

Seja $M=E \oplus E^{*}$, o K-espaço vetorial, objeto deste estudo, muni do da forma quadrática canônica $Q$ e a forma bilinear associada $B_{0}$.

Vimos que existe um isomorfismo linear $\sigma: E \rightarrow E^{*}$ tal que $\sigma(x)(y)=$ $=B_{1}(x, y) ;$ para $x, y \in E$.

Definimos a aplicação

$$
\begin{array}{ll}
\bar{\sigma}: E \rightarrow E \oplus E^{*} & \text { tal que } \\
\bar{\sigma}(x)=(x, \sigma(x)) & x \in E
\end{array}
$$

e representamos $M_{1}=\bar{\sigma}(E)$, a imagem de $E$ pela aplicação $\bar{\sigma}$.

$$
M_{1}=\{(x, \sigma(x)) \in M \quad \text { t.q. } \quad x \in E\}
$$

$M_{1} \bar{e}$ um subespaço de $M$ de dimensão $n$, que é isomorfo à $E$, através da aplicação $\bar{\sigma}$. 
Além disso, vale:

$$
\begin{aligned}
B_{0}(\bar{\sigma}(x), \bar{\sigma}(y)) & =B_{0}\left(\left(x, x^{\prime}\right),\left(y, y^{\prime}\right)\right)= \\
& =\frac{1}{2}\left[x^{\prime}(y)+y^{\prime}(x)\right]= \\
& =\frac{1}{2}[\sigma(x)(y)+\sigma(y)(x)]= \\
& =\frac{1}{2}\left[B_{1}(x, y)+B_{1}(y, x)\right]=B^{\prime}(x, y) \cdot x, y \in E
\end{aligned}
$$

Portanto, os espaços $\left(E, Q_{1}\right)$ e $\left(M_{1}, Q\right)$ são isométricos onde $Q_{1}$ é a forma quadrática associada a $B_{1}$.

Analogamente, define-se um isomorfismo linear:

$$
\begin{array}{ll}
\bar{\sigma}^{\prime}: E^{*} \rightarrow E \oplus E^{*} & \text { tal que } \\
\bar{\sigma}^{\prime}\left(x^{\prime}\right)=\left(\sigma^{-1}\left(x^{\prime}\right), x^{\prime}\right) & x^{\prime} \in E^{*}
\end{array}
$$

e representa-se $M_{2}=\bar{\sigma}^{\prime}\left(E^{*}\right)$.

$$
M_{2}=\left\{\left(\sigma^{-1}\left(y^{\prime}\right), y^{\prime}\right) \in M \quad \text { t.q. } \quad y^{\prime} \in E^{*}\right\}
$$

$M_{2}$ é um subespaço de $M$ de dimensão $n$, que é isomorfo $\bar{a} E^{*}$, através da aplicação $\bar{\sigma}^{\prime}$ Então vale:

$$
\begin{aligned}
B_{0}\left(\bar{\sigma}^{\prime}\left(x^{\prime}\right), \bar{\sigma}^{\prime}\left(y^{\prime}\right)\right) & =B_{0}\left(\left(x, x^{\prime}\right),\left(y, y^{\prime}\right)\right)= \\
& =\frac{1}{2}\left[x^{\prime}(y)+y^{\prime}(x)\right]= \\
& =\frac{-1}{2}\left[B_{2}\left(x^{\prime}, y^{\prime}\right)+B_{2}\left(y^{\prime}, x^{\prime}\right)\right]=B^{\prime \prime}\left(x^{\prime}, y^{\prime}\right) \\
& x^{\prime}, y^{\prime} \in E^{*}
\end{aligned}
$$


Portanto, os espaços $\left(E^{*}, Q_{2}\right)$ e $\left(M_{2}, Q\right)$ são isométricos, onde $Q_{2}$ é a forma quadrática associada a $\mathrm{B}_{2}$.

- Além disso, conclui-se que os espaços quadrāticos $\left(E^{*}, Q_{2}\right)$ são isométricos, através do isomorfismo linear $\sigma: E \rightarrow E^{*}$.

De fato:

$$
\begin{aligned}
B_{2}(\sigma(x), \sigma(y)) & =B_{2}\left(x^{\prime}, y^{\prime}\right)= \\
& =y^{\prime}(x)=\sigma(y)(x)=B_{1}(x, y)
\end{aligned}
$$

para $x, y \in E, \sigma(y)=y^{\prime}$ e $x=\sigma^{-1}\left(x^{\prime}\right)$.

Portanto, decompomos o espaço $M$, munido da forma quadrática canônica $Q$ em dois espaços quadráticos, $\left(E, Q_{1}\right)$ e $\left(E^{*}, Q_{2}\right)$, que são isométricos en tre sí.

Já definimos a àlgebra de Clifford do espaço $M$, em relação à forma quadrātica, que representamos por $C_{M^{*}}$. Podemos tambēm definir a á1gebra de Clifford do espaço $E$ em relação à forma quadrática $Q_{1}$, representada por $C_{E}$ e a álgebra de Clifford do espaço vetorial $\mathrm{E}^{*}$ em relação à forma quadrática $Q_{2}$, que serā representada $C_{E} *$.

Consideremos agora a álgebra $C_{E}$, àlgebra de clifford do espaço E em relação à forma quadrática $Q_{1}$; vale

$$
\begin{aligned}
& x^{2}=Q_{1}(x) \cdot 1 \\
& x y+y x=B_{1}(x, y) \cdot 1 \quad x, y \in E
\end{aligned}
$$

onde $B_{1}: E \times E \rightarrow K$ é a forma bilinear associada a $Q_{1}$, t.q.

$$
\begin{aligned}
& B_{1}(x, y)=x^{\prime}(y)=\sigma(x)(y)=\left\langle y, x^{\prime}\right\rangle \quad x^{\prime} \in E^{*} \\
& \text { ou } \quad B(x, y)=\frac{1}{2}\left[\left\langle y, x^{\prime}\right\rangle+\left\langle x, y^{\prime}\right\rangle\right]
\end{aligned}
$$




$$
\begin{aligned}
& \text { Sejam } x, y \in E \text { e }\left(x_{i}\right)_{1 \leq i \leq n} \text { base de } E, \\
& \quad x=\sum_{i=1}^{n} a_{i} x_{i}, \quad y=\sum_{j=1}^{n} b_{j} x_{j}
\end{aligned}
$$

e a igualdade (23) se escreve

$$
\sum_{i, j=1}^{n} a_{i} b_{j}\left(x_{i} x_{j}+x_{j} x_{i}\right)=\sum_{i, j=1}^{n} a_{i} b_{j} B_{1}\left(x_{i}, x_{j}\right) \cdot 1 \quad a_{i}, b_{j} \in k
$$

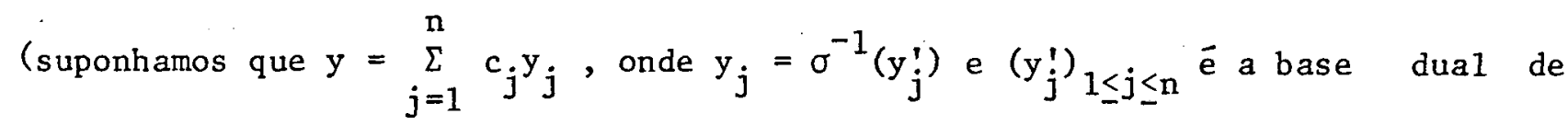
$\left.\left(x_{i}\right)_{1 \leq i \leq n}\right)$

'e então (24) se escreve

$$
\sum_{i, j=1}^{n} a_{i} c_{j}\left(x_{i} y_{j}+y_{j} x_{i}\right)=\sum_{i, j=1}^{n} a_{i} c_{j} B_{1}\left(x_{i}, y_{j}\right) \cdot 1 \quad a_{i}, c_{j} \in K
$$

e

$$
\begin{gathered}
B_{1}\left(x_{i}, y_{j}\right)=x_{i}^{\prime}\left(y_{j}\right)=y_{j}^{\prime}\left(x_{i}\right)=\delta_{i j} \quad\left(\text { se } \sigma=\sigma^{t}\right) \\
\text { Portanto, } \\
x_{i} y_{j}+y_{j} x_{i}=\delta_{i j} \cdot 1
\end{gathered}
$$

Definição 3.4. Chama-se produto interior (à direita) de um elemento $\mathrm{x} \in \Lambda E$ e um elemento $x^{\prime} \in \Lambda E^{*}$, e representa-se $x L x^{\prime}$, o valor parax do trans posto do endomorfismo $z^{\prime}+x^{\prime} \wedge z^{\prime}$ de $\Lambda E^{*}$.

Vale:

$$
\left\langle x L x^{\prime}, z^{\prime}\right\rangle=\left\langle x, x^{\prime} \wedge z^{\prime}\right\rangle
$$


Define-se o endomorfismo linear

$$
\begin{aligned}
& \tau\left(u^{\prime}\right): \Lambda E \rightarrow \Lambda E \quad u^{\prime} \in \Lambda E^{*} \\
& \tau\left(u^{\prime}\right)(x)=x L u^{\prime} .
\end{aligned}
$$

Prova-se como na página 65 que $\tau\left(y^{\prime}\right)$ é uma anti-derivação da ālge bra graduada $\Lambda \mathrm{E}$ se

$$
y^{\prime} \in E^{*} \quad x_{1}, \ldots, x_{p} \in E
$$

$\mathrm{Se}$

$$
\begin{aligned}
& v^{\prime} \in \stackrel{p-1}{\Lambda} E^{*} \text { e } y^{\prime}=x_{1}^{\prime} ; \quad v^{\prime}=x_{2}^{\prime} \wedge \cdots \wedge x_{i}^{\prime} \wedge \cdots \wedge x_{p}^{\prime} \\
& \left\langle\tau\left(y^{\prime}\right)\left(x_{1} \wedge \cdots \wedge x_{p}\right), v^{\prime}\right\rangle=\operatorname{det}\left|\left\langle x_{i}, x_{j}^{\prime}\right\rangle\right|
\end{aligned}
$$

Desenvolvendo este determinante em relação aos elementos da linha $i=1$, obtèm-se

$$
\begin{aligned}
\left\langle\tau\left(y^{\prime}\right)\left(x_{1} \wedge \cdots \wedge x_{p}\right), v^{\prime}\right\rangle= & \sum_{j=1}^{p}(-1)^{j+1}\left\langle x_{j}, x_{1}^{\prime}\right\rangle\left\langle x_{1} \wedge \cdots \wedge \hat{x}_{j} \wedge \cdots \wedge x_{p}, v^{\prime}\right\rangle \\
& \left(\text { se } x_{1}^{\prime}=y^{\prime}\right)
\end{aligned}
$$

Resulta que

$$
\begin{gathered}
\tau\left(y^{\prime}\right)\left(x_{1} \wedge \ldots \wedge x_{p}\right)=\sum_{j}(-1)^{j+1}\left\langle x_{j}, y^{\prime}>x_{1} \wedge \ldots \wedge \hat{x}_{j} \wedge \ldots \wedge x_{p}\right. \\
\text { Como } \tau\left(y^{\prime}\right) \text { abaixa o grau de uma unidade, temos }
\end{gathered}
$$

$$
\tau\left(y^{\prime}\right) \cdot w+w \cdot \tau\left(y^{\prime}\right)=0
$$

(w'è um automorfismo involutivo de $\Lambda E$ ); com a relação (1), isto prova que $\tau\left(y^{\prime}\right.$ ) è uma anti-derivação da álgebra graduada $\Lambda E$, (o sinal $\wedge$ acima de $x_{i}$ significa que o elemento $x_{i}$ é subraỉdo do produto). 
$\mathrm{Se}$

$$
y^{\prime} \in E^{*},\left[\tau\left(y^{\prime}\right)\right]^{2}=0
$$

Generalizamos os Teoremas 3.1 e 3.2 do seguinte modo:

(Teorema 3.5): "A älgebra $C_{M} \bar{e}$ isomorfa à älgebra End( $\left.\Lambda E\right)$ dos endomorfismos does paço vetorial $\Lambda \mathrm{E}$.

Prova: A álgebra exterior $\Lambda E$, que é a àlgebra de Clifford de $E$ (relativa à $Q_{\mid E}$ ), è considerada como sub-ālgebra de $C_{M}$, e vale:

$$
x \cdot y=x \wedge y \quad \forall x, y \in \Lambda E
$$

Seja

$$
\begin{gathered}
x \in E \text {, e } y^{\prime} \in E^{*} \text {. Temos: } \\
Q\left(x+y^{\prime}\right)=\left(x+y^{\prime}\right)^{2}=x^{2}+y^{\prime 2}+x y^{\prime}+y^{\prime} x=x y^{\prime}+y^{\prime} x= \\
=B\left(x, y^{\prime}\right) \cdot 1=\left\langle x, y^{\prime}\right\rangle \cdot 1=B_{1}(x, y)
\end{gathered}
$$

Definimos as aplicações:

$$
\rho_{1}: E \rightarrow \operatorname{End}(\Lambda E) \quad \rho_{2}: E^{*} \rightarrow \operatorname{End}(\Lambda E)
$$

1) Se $x \in E, \rho_{1}(x)$ é endomorfismo de $\Lambda E$ tal que

$$
\rho_{1}(x) u=x \wedge u \quad u \in \Lambda E
$$

2) Se $y^{\prime} \in E^{*}, \rho_{2}\left(y^{\prime}\right)$ é a anti-derivação de $\Lambda E$ tal que

$$
\rho_{2}\left(y^{\prime}\right)(x)=\left\langle x, y^{\prime}\right\rangle=B_{1}(x, y) \quad x, y \in E, \quad y^{\prime} \in E^{*}
$$


Observemos que a anti-derivação $\rho_{2}\left(y^{\prime}\right)=\tau\left(y^{\prime}\right)$ de $E$ é definida pelo produto interior (à direita) (ver Capítulo IV).

Usando a definição do produto interior (à direita), vemos que, se $u \in \stackrel{p}{\Lambda}, u=x_{1} \wedge \cdots \wedge x_{p}$, então $\rho_{2}\left(y^{\prime}\right): \Lambda E \rightarrow \Lambda E$ é um endomorfismo linear tal que $\rho_{2}\left(y^{\prime}\right)(u)=\tau\left(y^{\prime}\right)(u)=\underset{j}{\sum}(-1)^{j+1}<x_{j}, y^{\prime}>x_{1} \wedge \ldots \wedge x_{j-1} \hat{x}_{j} \wedge x_{j+1} \wedge \ldots \wedge x_{p}$ Se $u \in \stackrel{p}{\Lambda E}, \quad v \in \stackrel{q}{\Lambda}$,

$$
\rho_{2}\left(y^{\prime}\right)(u v)=\left[\rho_{2}\left(y^{\prime}\right) u\right] v+(-1)^{p} \cdot u\left[\rho_{2}\left(y^{\prime}\right) v\right]
$$

Resu1ta, por recorrência, que, se u é homogêneo de grau $p, \rho_{2}\left(y^{\prime}\right) u$ é homogêneo de grau $(p-1)$, o que implica $\rho_{2}\left(y^{\prime}\right)^{2}=0$.

A aplicação linear $\rho: E \oplus E^{*}=M \rightarrow E n d(\Lambda E)$ é definida por:

$$
\rho\left(x+y^{\prime}\right)=\rho_{1}(x)+\rho_{2}\left(y^{\prime}\right)
$$

e

$$
\begin{gathered}
{\left[\rho\left(x+y^{\prime}\right)\right]^{2}=\rho_{1}^{2}(x)+\rho_{2}\left(y^{\prime}\right)+\rho_{1}(x) \rho_{2}\left(y^{\prime}\right)+\rho_{2}\left(y^{\prime}\right) \cdot \rho_{1}(x)=} \\
=\rho_{1}(x) \cdot \rho_{2}\left(y^{\prime}\right)+\rho_{2}\left(y^{\prime}\right) \cdot \rho_{1}(x) \\
\text { Calculemos o valor deste endomorfismo de } \Lambda E: \\
\rho_{2}\left(y^{\prime}\right)\left[\rho_{1}(x) \cdot u\right]=\rho_{2}\left(y^{\prime}\right)[x \wedge u]=\rho_{2}\left(y^{\prime}\right) x \wedge u-x \wedge \rho_{2}\left(y^{\prime}\right) u= \\
<x, y^{\prime}>\cdot u-x \wedge \rho_{2}\left(y^{\prime}\right) u=Q\left(x+y^{\prime}\right) \cdot u-\rho_{1}(x) \cdot \rho_{2}\left(y^{\prime}\right) \cdot u \quad u \in E
\end{gathered}
$$




\section{Portanto:}

$$
\left[\rho_{1}(x) \cdot \rho_{2}\left(y^{\prime}\right)+\rho_{2}\left(y^{\prime}\right) \rho_{1}(x)\right](u)=Q\left(x+y^{\prime}\right) \cdot u \quad u \in E
$$

e

$$
\rho\left(x+y^{\prime}\right)^{2}=Q\left(x+y^{\prime}\right) \cdot I=Q(z) \cdot I \quad \text { se } z=x+y^{\prime} \in M
$$

Resulta, da propriedade universal das álgebras de Clifford, que a aplicação $\rho$ se estende em um homomorfismo de álgebras unitárias

$$
\phi: C_{M} \rightarrow \operatorname{End}(\Lambda E)
$$

Como $\operatorname{dim} C_{M}=2^{2 n}=\operatorname{dim} \operatorname{End}(\Lambda E)$, para mostrar que $\phi \bar{e}$ um isomorfismo, falta provar que $\phi \bar{e}$ sobrejetora.

$$
\text { Seja e }=\left(e_{1}, \ldots, e_{n}\right) \text { uma base de E e } e^{\prime}=\left(e_{1}^{\prime}, \ldots, e_{n}^{\prime}\right) \text { a base de }
$$

$E^{*}$ dual de e

$$
\left[\left\langle x_{i}, x_{j}^{\prime}\right\rangle=\delta_{i j}\right]
$$

Sabemos que $E$ admite uma base formada de $2^{n}$ elementos $e_{H}$, onde $\mathrm{H}$ percorre o conjunto de todas as partes do intervalo $[1, n]$ de $\mathbb{N}$.

A multiplicação é dada por

$$
\begin{array}{ll}
e_{H} \wedge e_{K}=0 & \text { se } H \cap K \neq \phi \\
e_{H} \wedge e_{K}=\rho_{H, K} e_{H} \cup \mathrm{K} & \text { se } H \cap K=\phi
\end{array}
$$

com $\rho_{H, K}=(-1)^{v}$, v sendo o nümero de pares $(i, j)$ tal que $i \in H, j \in K$ e $j<i$. 
Seja $J$ uma seqlência estritamente crescente de índices entre 1 e $\mathrm{n}: 1 \leq \mathrm{j}_{1}<\mathrm{j}_{2}<\ldots<\mathrm{j}_{\mathrm{p}} \leq \mathrm{n}, \mathrm{R}$ a seqUência $(1,2, \ldots, \mathrm{n}), \mathrm{J}^{\prime}=\mathrm{R}-\mathrm{J}$, representa-se

$$
e_{J}=e_{j_{1}} \cdots e_{j_{p}} ; e_{J}^{\prime}=e_{j_{1}}^{\prime} \cdots e_{j_{p}}^{\prime}
$$

Temos:

$$
\text { (2) } \begin{cases}\phi\left(e_{J}^{\prime}\right) \cdot e_{L}=e_{L} \tau e_{J}^{\prime}=0 & \text { se } J \not L L \\ \phi\left(e_{J}^{\prime}\right) \cdot e_{L}=e_{L} \tau e_{J}^{\prime}= \pm e_{L-J} & \text { se } J \subset L\end{cases}
$$

(2) resulta do cálculo do produto interior à direita nos elementos da base de $\Lambda E[5]$.

$$
\text { (3) } \begin{cases}\phi\left(e_{J}\right) \cdot e_{L}=e_{J} \wedge e_{L}= \pm e_{J} \cup L & \text { se } J \cap_{L}=\phi \\ \phi\left(e_{J}\right) \cdot e_{L}=0 & \text { se } J \cap L \neq \phi\end{cases}
$$

(3) resulta da multiplicação em $\Lambda E$ de elementos da base [5].

Representa-se

$$
a_{I J}=\phi\left(e_{I}\right) \phi\left(e_{R}^{\prime}\right) \phi\left(e_{J}\right)=\phi\left(e_{I} e_{R}^{\prime} e_{J}\right)
$$

e obtém-se:

$$
\text { (4) } \begin{cases}a_{I J}\left(e_{J}\right)= \pm e_{I} \\ a_{I J}\left(e_{L}\right)=0 & \text { se } L \neq J \quad\left(e_{L} \in \text { base de } \Lambda E\right)\end{cases}
$$


Conclui-se que $\phi \bar{e}$ um isomorfismo, pois os endomorfismos definidos acima (4) constituem uma base de End( $\Lambda E)$.

OBS: A prova deste teorema implica também na afirmação feita anteriormente, que a àlgebra de Clifford $C_{M} \bar{e}$ isomorfa à álgebra de matrizes $K\left(2^{n}\right)$. 


\section{CAPİTULO IV}

\section{AS ÁLGEBRAS DE CLIFFORD COMO DEFORMACÕES DAS ÁLGEBRAS EXTERIORES}

o objetivo deste capítulo é mostrar as relações entre a álgebra de Clifford $C(E, Q)$ do espaço quadrātico $Q$ e a ālgebra, $\Lambda E$, álgebra exterior de $E$, utilizando-se de resultados anteriores obtidos através do estudo da álgebra $\mathrm{C}_{\mathrm{M}}$. Un resultado já estabelecido. é que os espaços vetoriais $C(E, Q)$ e $\Lambda E$ são isomorfos [8]; Chevalley mostra também que a multiplicação em $C(E, Q)$ pó de ser obtida da multiplicação em $\Lambda E$ e da forma $B_{0}$, como já foi explicitado.

0 autor J. Helmstetter [17], se propõe a obter as àlgebras de Clifford como deformações das älgebras exteriores. Para isso, utiliza sistematicamente as técnicas de produtos interiores e abandona as técnicas clássicas das àlgebras de Clifford; esse procedimento é mais adequado aos resultados que se pre tende obter e pode ser utilizado com poucas mudanças na àlgebra de Weyl.

Obtém-se sobre o espaço vetorial $\Lambda E$ um produto (que depende da forma bilinear $B)$, que a transforma numa álgebra $\Lambda(E, B)$ isomorfaààlgebra $C(E, Q)$. Reciprocamente, conhecendo-se a álgebra $\Lambda(E, B)$, é possível, gra ças à propriedade universal da ālgebra de Clifford, reencontrar o isomorfismo $\theta_{B}$ (isomorfismo entre os espaços vetoriais $\mathrm{C}(\mathrm{E}, \mathrm{Q})$ e $\Lambda \mathrm{E}$ e temos $\theta_{\mathrm{B}}(\mathrm{a})$ = a para todo $a \in \mathrm{E})$.

Este raciocínio permite utilizar e estudar estes isomorfismos $\theta_{B}$, relacionados às formas bilineares $B: E \times E \rightarrow K,(t \cdot q \cdot B(a, a)=Q(a) \forall a \in E) o$ que é uma generalização em relação ao estudo de Chevalley, que só usa a forma bị linear canonicamente associada a $Q$.

Fundamentando-se no raciocínio de Chevalley e utilizando os pro dutos interiores e os resultados de J.H., mostra-se que uma álgebra de Clifford pode ser obtida como uma "deformação" da ālgebra exterior $\Lambda E$; e finalmente, como isto pode ser relacionado com os resultados que obtivemos. 
Seja E um K-espaço vetorial de dimensão $n$.

Definição 4.1. Seja $\left(x_{1}, \ldots, x_{n}\right.$ base de E. Se

$$
u=\sum_{i<j} a_{i j} x_{i} \wedge x_{j}
$$

é um elemento de $\stackrel{2}{\Lambda} E$, define-se

$$
\exp u=\prod_{i<j}\left(1+a_{i j} x_{i} \wedge x_{j}\right)
$$

Verifica-se que:

$$
\exp \left(u+u^{\prime}\right)=(\exp u) \wedge\left(\exp u^{\prime}\right) \quad u, u^{\prime} \in \stackrel{2}{\Lambda} E
$$

$\mathrm{Se}$

$$
u=a x_{k} x, x=\sum_{i=1}^{r} a_{i} x_{i} \text {, ou se }
$$

u é um elemento decomponíve1, temos

$$
\exp u=1+u
$$

Isto mostra que a definição de exp u não depende da base especial escolhida em E.

Vale

$$
\exp 0=1
$$

o que implica que exp u è sempre inversível e que

$$
(\exp u)^{-1}=\exp (-u)
$$

Para todo $u \in \stackrel{2}{\Lambda} E$, exp u estä em $\Lambda_{+}$E e difere de $1+$ u por um ele mento de

$$
\sum_{\mathrm{k}>1}^{2 \mathrm{k}} \Lambda \mathrm{E}
$$


Introduz-se as notações, relativas as älgebras graduadas sobre $\mathbb{Z} / 2 \mathbf{Z}$.

A aplicação $x \rightarrow \tilde{x} \bar{e}$ o automorfismo $\Lambda E \rightarrow \Lambda E$ t.q. $\tilde{x}=x$.se, $x \quad e ́$ par e $\tilde{x}=-x$ se $x \bar{e}$ ímpar.

Se $\mathrm{x}$ e ye $\Lambda \mathrm{E}$, coloca-se:

$$
\begin{aligned}
& \sigma(x, y)=1 \text { se } x \text { ou } y \text { é par } \\
& \sigma(x, y)=-1 \text { se } x \text { e y são impares }
\end{aligned}
$$

Definição 4.2. Chama-se produto interior (à) direita de um elemento $\mathrm{x} \in \Lambda \mathrm{E}$ e de um elemento $x^{\prime} \in \Lambda E^{*}$, e representa-se $x L x^{\prime}$ (ou $x \tau x^{\prime}$ ), o valor para $\mathrm{x}$ do transposto do endomorfismo

$$
z^{\prime}+x^{\prime} \wedge z^{\prime} \text { de } \Lambda E^{*}
$$

Vale identicamente:

$$
\left\langle x L x^{\prime}, z^{\prime}\right\rangle=\left\langle x, x^{\prime} \wedge z^{\prime}\right\rangle
$$

Verifica-se que a adição e a lei de composição externa $\left(x^{\prime}, x\right) \rightarrow$ $\rightarrow \mathrm{x} L \mathrm{x}^{\prime}$ sobre $\Lambda \mathrm{E}$ definem sobre este conjunto uma estrutura de módulo à direita, em relação ao anel $\Lambda E^{*}$. Temos a identidade

$$
x\left\llcorner\left(x^{\prime} \wedge y^{\prime}\right)=\left(x<x^{\prime}\right) L y^{\prime}\right.
$$

Deduz-se, em particular que, para um p-vetor $x$ e uma q-forma $x^{\prime}$,

temos

$$
\begin{array}{lr}
x\left\llcorner x^{\prime}=0\right. & p<q \\
x L^{\prime}=(p-q) \text {-vetor } & \text { se } p \geq q \\
x\left\llcorner x^{\prime}=\left\langle x, x^{\prime}\right\rangle\right. & \text { se } p=q
\end{array}
$$


Identifica-se o dual de $\stackrel{p}{\Lambda}$ E a $\stackrel{p}{\Lambda}{ }^{*}$ e a forma bilinear canônica so bre $\stackrel{p}{(\Lambda E}) \times\left(\stackrel{p}{\Lambda} E^{*}\right)$ è definida pela fórmula fundamental

$$
\left\langle x_{1} \wedge \cdots \wedge x_{p}, x_{i}^{\prime} \wedge x_{2}^{\prime} \wedge \cdots \wedge x_{p}^{\prime}\right\rangle=\operatorname{det}\left(\left\langle x_{i}, x_{j}^{\prime}\right\rangle\right)
$$

Definição 4.3. "Chama-se produto interior (ā) esquerda de um elemento $\mathrm{x} \in \Lambda \mathrm{E}$ e de um elemento $x^{\prime} \in \Lambda E^{*}$, e representa-se $x \_\mid x^{\prime}$, o valor para ' $x$ ' da transposta do endomorfismo $z \rightarrow z \wedge x$ de $\Lambda E . "$

Temos a identidade

$$
\left\langle z, x \mid x^{\prime}\right\rangle=\left\langle z \wedge x, x^{\prime}\right\rangle \quad x, z \in \Lambda E, x^{\prime} \in \Lambda E^{*}
$$

Proposição 4.4.[5]"A adição e a lei de composição externa $\left.\left(x, x^{\prime}\right) \rightarrow x\right\rfloor x^{\prime}$ sobre $\Lambda E^{*}$ definem sobre este conjunto uma estrutura de módulo à esquerda em relação ao anel $\Lambda \mathbb{E}$."

Conclui-se na demonstração desta Proposição, que

$$
\left.(x \wedge y)\left\lfloor x^{\prime}=x\right\rfloor(y\rfloor x^{\prime}\right)
$$

para $x, y \in \Lambda E, x^{\prime} \in \Lambda E^{*}$.

$$
\begin{array}{ll}
\text { Deduz-se que se } x \in \stackrel{p}{\Lambda E} \text { e } x^{\prime} \in \stackrel{q}{\Lambda *}, \\
x\rfloor x^{\prime}=0 & \text { se } p>q \\
x\rfloor x^{\prime}=(q-p) \text {-forma } & \text { se } p \leq q \\
x\rfloor x^{\prime}=\left\langle x, x^{\prime}\right\rangle & \text { se } p=q
\end{array}
$$

OBS:- A seguir usaremos a notação $x \tau y^{\prime}$ (ou $x^{\prime} \tau$ y) para representar o produto interior à direita (ou à esquerda)

$$
x, y \in \Lambda E \text { e } x^{\prime}, y^{\prime} \in \Lambda E^{*}
$$


Definição 4.5. "Seja A uma älgebra e seja w um automorfismo involutivo de A. Uma derivação $\theta$ de A (relativamente à $\omega$ ) é um endomorfismo 1inear de A tal que

$$
\theta \omega-\omega \theta=0 \text { e } \theta(a b)=(\theta . a) b+a(\theta . b) \quad a, b \in A
$$

Uma anti-derivação $\bar{\theta}$ de A (relativamente à $\omega$ ) è um endomorfismo linear de A tiq.

$$
\bar{\theta} \omega+\omega \bar{\theta}=0 \text { e } \bar{\theta}(a b)=(\bar{\theta} a) b+(\omega . a)(\bar{\theta} b) \quad a, b \in A^{\prime \prime} .
$$

Se A possui um elemento unidade 1 , observa-se que $1 \bar{e}^{-}$um zero de todas as derivações e anti-derivações de A.

Seja $E$ espaço vetorial de dimensão $n$; a potência exterior $\stackrel{n}{\Lambda E}$ tem uma base formada de um só elemento.

Proposição 4.6. [5]"Seja o um elemento de $\Lambda E$ formando uma base deste espaço, e' o elemento de $\Lambda^{n}{ }^{*}$ formando a base dual de e. A aplicação $\mathrm{x} \rightarrow \mathrm{x}\rfloor \mathrm{e}^{\prime}$ é um isomorfismo de $\Lambda$ E. sobre $\Lambda E^{*}$, que aplica $\Lambda \mathrm{E}$ sobre $\Lambda \mathrm{E}^{*}$ para $0 \leq \mathrm{p} \leq \mathrm{n}$; o isomorfismo inverso é a aplicação $x^{\prime} \rightarrow$ e L $x^{\prime} . "$

Considerando-se o produto interior à esquerda definido anteriormente $(x \in \Lambda E)$ temos um endomorfismo de $\Lambda E^{*}$, que representaremos for

$$
\begin{array}{ll}
\tau(x): \Lambda E^{*} \rightarrow \Lambda E^{*} \quad \text { t.q. } & \\
\left.\tau(x)\left(x^{\prime}\right)=x\right\rfloor x^{\prime} & x \in \Lambda E, x^{\prime} \in \Lambda E^{*}
\end{array}
$$

Quando $x \in E$, o endomorfismo $\tau(x)$ se explicita simplesmente. Su ponhamos que

$$
x_{1}^{\prime}, \ldots, x_{p}^{\prime} \in E^{*} \quad \text { e } \quad y \in E
$$


$\mathrm{p}-1$

Para todo elemento $v \in \Lambda E$, decomponível em $v=x_{2} \wedge \ldots \wedge x_{i} \wedge . . \wedge x_{p}$, temos, colocando $x_{i}=y$

$$
\left\langle v, \tau(y)\left(x_{1}^{\prime} \wedge \ldots \wedge x_{j}^{\prime} \wedge \ldots \wedge x_{p}^{\prime}\right)\right\rangle=\operatorname{det} \mid\left\langle x_{i}, x_{j}^{\prime}>\right|
$$

Desenvolvendo o determinante em relação aos elementos da linha $i=1$, obtēm-se

$$
\left\langle v, \tau(y)\left(x_{1}^{\prime} \wedge . . x_{j}^{\prime} \ldots x_{p}^{\prime}\right)>=\sum_{j=1}^{p}(-1)^{j+1}\left\langle x_{1}, x_{j}^{\prime}>\left\langle v, x_{1}^{\prime} \wedge \ldots \hat{x}_{j}^{\prime} \ldots x_{p}^{\prime}\right\rangle\right.\right.
$$

Resulta que

$$
\begin{gathered}
\tau(y)\left(x_{1}^{\prime} \wedge . . x_{j}^{\prime} \ldots \wedge x_{p}^{\prime}\right)=\sum_{j=1}^{p}(-1)^{j+1}<y, x_{j}^{\prime}>x_{1}^{\prime} \wedge \ldots x_{j}^{\prime} \wedge \ldots \wedge x_{p}^{\prime} \\
\text { Como } \tau(y) \text { abaixa o grau de uma unidade, temos }
\end{gathered}
$$

$$
\tau(y) \omega+\omega \tau(y)=0 ;
$$

De (2) e (3) conclui-se que $\tau(y)$ è uma anti-derivação da álge bra graduada $\Lambda E^{*}$.

Analogamente, considerando-se o produto interior à direita e $y^{\prime} \in \Lambda E^{*}$, mostra-se que

$$
\begin{gathered}
\tau\left(y^{\prime}\right): \Lambda E \rightarrow \Lambda E \quad \text { t.q. } \\
\tau\left(y^{\prime}\right)(x)=x L y^{\prime} \quad\left(\text { ou } x \tau y^{\prime}\right) \quad x \in \Lambda E
\end{gathered}
$$

è um endomorfismo linear e se $\dot{y}^{\prime} \in \mathrm{E}^{*}, \underline{\tau\left(y^{\prime}\right)}$ é uma anti-derivação da àlgebra gra duada $\Lambda$ E.

Definição 4.7. A e B são duas álgebras graduadas, o produto tensorial graduado de $A$ e $B$, repres. A $\hat{Q} B, \bar{e}$ uma á1gebra graduada, cuja multiplicação em A ̂ิ B é dada por

$$
(a \otimes b)\left(a^{\prime} \otimes b^{\prime}\right)=\sigma\left(b, a^{\prime}\right) \quad a a^{\prime} \otimes b b^{\prime}
$$


Podemos citar outras propriedades do produto interior (p.i.d):

$$
\begin{aligned}
& \text { se } \quad u \in \stackrel{2}{\Lambda} \text { e } b^{\prime} \in E^{*} \\
& (\operatorname{Exp} u) \tau b^{\prime}=\left(u \tau b^{\prime}\right) \wedge \operatorname{Exp} u
\end{aligned}
$$

Se $f$ é uma aplicação linear de $E$ em um K-espaço vetorial F, e $\bar{f} \bar{e}$ o seu prolongamento canônico $\Lambda E$ em $\Lambda$, vale:

$$
\bar{f}(x) \tau z^{\prime}=\bar{f}\left(x \tau{ }^{t} \bar{f}\left(z^{\prime}\right)\right) \quad \text { se } x \in \Lambda E \text { e } z^{\prime} \in \Lambda F^{*}
$$

Além disso, identifica-se $\Lambda(E \oplus F)$ com $\Lambda E \widehat{Q} \Lambda F$, no qual o produ to exterior é definido

$$
\begin{aligned}
& \quad\left(x \otimes x^{\prime}\right) \wedge\left(y \otimes y^{\prime}\right)=\sigma\left(x^{\prime}, y\right) \cdot(x \wedge y) \otimes\left(x^{\prime} \wedge y^{\prime}\right) . \\
& \text { se } \quad x, y \in \Lambda E \text { e } x^{\prime}, y^{\prime} \in \Lambda F
\end{aligned}
$$

Também identifica-se todo $(\mathrm{a}, \mathrm{b}) \in \mathrm{E} \oplus \mathrm{F}$ com $\mathrm{a} \otimes 1+1 \otimes \mathrm{b} \in \Lambda \mathrm{E} \otimes \Lambda \mathrm{F}$. Se $x \in \Lambda E, x^{\prime} \in \Lambda E^{*}, y \in \Lambda F$ e $y^{\prime} \in \Lambda F^{*}$, pode-se calcular o pro duto interior de $x \otimes y \in \Lambda(E \oplus F)$ por $x^{\prime} \otimes y^{\prime} \in \Lambda(E \oplus F)^{*}$ :

$$
(x \otimes y) \tau\left(x^{\prime} \otimes y^{\prime}\right)=\sigma\left(x \tau x^{\prime}, y^{\prime}\right) \cdot\left(x \tau x^{\prime}\right) \otimes\left(y \tau y^{\prime}\right)
$$

Em particular, se $x \otimes x^{\prime} \in \Lambda\left(E \oplus E^{*}\right)$ e $y^{\prime} \otimes y \in \Lambda\left(E \oplus E^{*}\right)^{*} \cong$ $\cong \Lambda\left(\mathrm{E}^{*} \oplus \mathrm{E}\right)$, fica:

$$
\left(x \otimes x^{\prime}\right) \tau\left(y^{\prime} \otimes y\right)=\sigma\left(x \tau y^{\prime}, y\right)\left(x \tau y^{\prime}\right) \otimes\left(x^{\prime} \tau y\right)
$$

A seguir, estabeleceremos a relação fundamental entre a ālgebra de Clifford de um espaço quadrático E e a álgebra exterior do espaço vetorial E, segundo Chevalley. 
Retornamos ao homomorfismo $\psi: \mathrm{C}_{\mathrm{E}} \rightarrow$ End $(\Lambda E)$, definido no Teorema 1.16, onde $\psi \bar{e}$ a extensão à $C_{E}$ da aplicação linear

$$
\begin{aligned}
& \varphi: E \rightarrow \text { End }(\Lambda E) \text { t.q. } \\
& x \rightarrow L_{x}^{\prime} \\
& \text { e } \quad L_{x}^{\prime}(u)=L_{x} u+\delta_{x} \cdot u \quad u \in C_{E}
\end{aligned}
$$

onde $L_{x}(u)=x \wedge u \bar{e}$ a multiplicação à esquerda por $x$ em $\Lambda E$ e $\delta_{x} \bar{e}$ uma anti-derivação de $\Lambda E$ t.q.

$$
\delta_{x}(y)=B(x, y) \quad x, y \in E
$$

Em particular, se $z=x+x^{\prime} \in M=E \oplus E^{*}$, representamos

$$
L_{1 z}^{\prime}(y)=L_{x} \cdot y+\delta_{x^{\prime}} \cdot y
$$

onde $\delta_{x^{\prime}}: \Lambda E \rightarrow \Lambda E \bar{e}$ a anti-derivação $t \cdot q \cdot \delta_{x^{\prime}}(y)=x^{\prime}(y)=B(x, y)$.

Temos o seguinte

Teorema 4.8. [8] "Seja uma forma bilinear $B_{0}$ em $E \times E$ tal que $Q(x)=B_{o}(x, x)$ para $x \in E$. Podemos então identificar o espaço vetorial "subjacen te" da álgebra de Clifford $C_{E}$ com o da ālgebra exterior $\Lambda E$ de mo do que, para todo $x \in E$, o operador da multiplicação à esquerda por $x$ em $C_{E} \bar{e} L_{x}+\delta_{x}$, onde $L_{x}$ é o operador da multiplicação à esquerda por $\mathrm{x}$ em $\Lambda E$ e $\delta_{\mathrm{x}}$ a anti-derivação de $\Lambda E$ tal que

$$
\delta_{x} \cdot y=B_{o}(x, y) \cdot 1 \quad \text { para } \quad y \in E
$$

Seja $C_{E}^{h}$ o subespaço de $C_{E}$ gerado pelos produtos de no máximo $h$ elementos de $E$ e $\Lambda E$ o espaço dos elementos homogêneos de grau $h$ de $\Lambda E$; então temos

$$
C_{E}^{h}=\sum_{h^{\prime} \leq h} h^{\prime} \Lambda E
$$


Se $x_{1}, \ldots, x_{h}$ estão em $E$, então temos para $h \geq 2$,

$$
x_{1} \cdots x_{h} \equiv x_{1} \wedge \cdots \wedge x_{h}\left(\bmod c_{E}^{h-2}\right)
$$

Temos

$$
\mathrm{C}_{\mathrm{E}}^{+}=\sum_{\mathrm{h} \text { par }} \Lambda_{\mathrm{E}}^{\mathrm{h}}, \mathrm{C}_{\mathrm{E}}^{-}=\sum_{\mathrm{h} \text { impar }} \Lambda_{\mathrm{E}}^{\mathrm{h}} \text {. }
$$

\section{Prova}

Coloca-se $\theta(u)=\psi(u)(1)$ para $u \in C_{E}$.

Então $\theta$ é uma aplicação linear de $C_{E}$ em $\Lambda E$.

Lembremos que um elemento $\Lambda \in$ End $(\Lambda E)$ é chamado homogêneo de grau d se $\Lambda$ leva todo elemento homogêneo de grau $h$ de $\Lambda E$ em um elemento homogêneo de grau $h+d$.

Se $\Lambda_{1}, \ldots, \Lambda_{h}$ são homogêneos de graus $d_{1}, \ldots, d_{h}$ então $\Lambda_{1} \ldots \Lambda_{h}$ è homogêneo de grau $d_{1}+\ldots+d_{h}$.

Para todo $x \in E, L_{x}$ é homogêneo de grau 1 e $\delta_{x}$ de grau -1 .

Seja $x_{1}, \ldots, x_{h} \in E$. Então temos

$$
\psi\left(x_{1} \ldots x_{h}\right)=\left(L_{x_{1}}+i_{x_{1}}\right) \ldots\left(L_{x_{h}}+i_{x_{h}}\right)
$$

e isto pode ser escrito como

$$
\psi\left(x_{1} \ldots x_{h}\right)=L_{x_{1}} \cdots L_{x_{h}}+\sum_{d=-h}^{h-1} \Lambda_{d}
$$

onde $L_{d}$ é homogêneo de grau d.

Segue que

$$
\theta\left(x_{1} \ldots x_{h}\right)=x_{1} \wedge \cdots \wedge x_{h}+\sum_{d=0}^{h-1} \xi_{d}
$$

onde $\xi_{d} \bar{e}$ homogêneo de grau d. 
Desde que $\operatorname{dim} C_{E}=2^{n}=\operatorname{dim} \Lambda E, \theta$ definida acima $\bar{e}$ um isomorfis mo linear que coincide com a identidade em K.l e em E.

Identificaremos o espaço vetorial subjacente de $C_{E}$ com o de $\Lambda E$ por meio de $\theta$.

Se $u, v \in \Lambda E$, u.v representa seu produto em $C_{E}$, enquanto $u \wedge . v$ é seu produto em $\Lambda \mathbb{E}$. Devemos observar que essa identificação depende da escolha de uma forma bilinear $B_{0}$. Tal que $B_{0}(x, x)=Q(x)$.

Observemos que, na fórmula (7) acima, $\Lambda_{d} \bar{e}$ diferente de zero se $d \equiv h(\bmod 2)$, porque um produto de $r$ operadores $L_{x_{i}}$ e $h-r$ operadores $\delta_{x_{i}} \bar{e}$ de grau $h-2 r$.

Por outro lado, $C_{E}^{+}$(respectivamente: $C_{E}^{-}$) é obviamente gerado pe los produtos de um número par (respectivamente: ímpar) de fatores em M. Segue que $C_{+}$(respectivamente: $C_{-}$) è o conjunto de elementos de $\Lambda E$ cujos componentes ho mogêneos $\neq 0$ são todos de grau par (respectivamente: impar).

Isto mostra que a involução principal de $C_{E} \vec{e}$ a mesma que a de $\Lambda$ E. Além disso, vemos que

$$
\mathrm{x}_{1} \ldots \mathrm{x}_{\mathrm{h}} \equiv \mathrm{x}_{1} \wedge \cdots \wedge \mathrm{x}_{\mathrm{h}}\left(\bmod \sum_{h^{\prime} \leq h-2} \stackrel{h}{\prime}^{\prime} \mathrm{E}^{\prime}\right)
$$

para todo $x_{1}, \ldots, x_{h} \in E$.

Mais explicitamente:

$$
x_{1} x_{2} \cdots x_{h}=x_{1} \wedge \cdots x_{h}+x_{h-2}+x_{h-4}+\ldots+y
$$

onde

$$
\begin{aligned}
X_{h-i} \in \stackrel{h-i}{\Lambda} ; \quad Y \in \stackrel{\circ}{\Lambda} \cong \mathrm{K} \text { se } \mathrm{h} \text { ē par } \\
\text { e } \mathrm{Y} \in \stackrel{1}{\Lambda \mathrm{E} \cong \mathrm{E}} \text { se } \mathrm{h} \text { ē impar }
\end{aligned}
$$


Através destes resultados, o autor [8] estabelece a relação fun damental entre as ālgebras de Clifford $C_{E}$ e a álgebra exterior $\Lambda E$,

$$
x y=x \wedge y+B(x, y) \quad x, y \in E
$$

onde $\mathrm{B}$ é a forma bilinear associada a Q.

Baseando-se no trabalho de J.Helmstetter e utilizando-se os produ tos interiores, obteve-se uma generalização de (9), na qual o isomorfismo $\theta_{B}$ entre os espaços vetoriais $C_{E}$ e $\Lambda E$ depende de uma forma bilinear $B$ t.q.

$$
B(x, x)=Q(x)
$$

para todo $x \in E$ e essa forma bilinear não é necessariamente a forma bilinear associada a $Q$.

Uma das vantagens desse procedimento é que ele pode ser utiliza do com poucas mudanças na álgebra simplética (ou álgebra de Wey1).

Sejam $\mu: E \oplus E \rightarrow E, \delta: E \rightarrow E \oplus E$ e $T: E \oplus E \rightarrow E \oplus E$ as aplicações lineares definidas por:

$$
\mu(a, b)=a+b, \delta(a)=(a, a) \text { e } T(a, b)=(b, a) \quad a, b \in E
$$

Representa-se $\bar{\mu}, \bar{\delta}$ e $\bar{T}$ seus prolongamentos em homomorfismos das ālgebras exteriores; como identifica-se $\Lambda(E \oplus E)$ com $\Lambda E \hat{E}$, pode-se escrever:

$$
\bar{\mu}(x \otimes y)=x \wedge y \text { e } \bar{T}(x \otimes y)=\sigma(x, y) \cdot y \otimes x \text { se } x, y \in \Lambda E
$$

Sejam $\mu^{\prime}, \delta^{\prime}$ e $\mathrm{T}^{\prime}$ as aplicações em $\mathrm{E}^{*}$ definidas de modo anālogo e $\bar{\mu}^{\prime}, \bar{\delta}^{\prime}$ e $\bar{T}^{\prime}$ seus prolongamentos a $\Lambda E^{*}$.

Observemos que as aplicações $\mu^{\prime}, \delta^{\prime}, T^{\prime}$ relativas a $E^{*}$ são res pectivamente as transpostas das aplicações $\delta, \mu, \tau$ relativas a $E$, o que permite a aplicação de (5). 


$$
\begin{gathered}
\text { Se } f: \Lambda E \rightarrow \Lambda F^{*} \text { e se } x \in \Lambda E \text { e } z^{\prime} \in \Lambda F^{*} \\
f(x) \tau z^{\prime}=f\left(x \tau_{f\left(z^{\prime}\right)}\right.
\end{gathered}
$$

Seja $B: E \times E \rightarrow K$ uma forma bilinear. Existe um isomorfismo en tre $B(E)$ (formas bilineares sobre $E$ ) e $E^{*} E^{*} t . q$.

$$
\begin{aligned}
& B \leftrightarrow x^{\prime} y^{\prime} \text { se e somente se } \\
& \quad\left(x^{\prime} y^{\prime}\right)(a, b)=x^{\prime}(a) \cdot y^{\prime}(b)=B(a, b) \text { a, } b \in E
\end{aligned}
$$

Teorema 4.9. [9] "Seja E um espaço vetorial de dimensão finita, B uma forma bili near simétrica sobre $E \times E, E^{*} \circ$ dual de $E$ e $B$ a aplicação $1 i-$ near de $E$ em $E^{*}$ canonicamente associada à $B$. Prolonguemos $B$ em um homomorfismo unitário $\beta$ da ālgebra tensorial $T(E)$ na ālgebra tensorial $\mathrm{T}\left(\mathrm{E}^{*}\right)$; representamos $\mathrm{T}_{2}(\mathrm{E})$ e $\mathrm{T}_{2}\left(\mathrm{E}^{*}\right)$ os espaços de elementos homogêneos de grau 2 de $T(E)$ e $T\left(E^{*}\right)$.

A forma $B$ pode então ser considerada como um elemento de $T_{2}\left(E^{*}\right)$. Como tal, $B$ pertence à imagem $\beta\left(T_{2}(E)\right)$ de $T_{2}(E)$ por $B$. Pode-se também considerar a forma bilinear B como definindo uma função linear, que representaremos ainda por B sobre E E. Seja p a dimensão do subespaço $\beta(E)$ de $E^{*}$. Se $t \in E \otimes E^{-}$tal que $B(t)=B$, temos $B(t)=p \cdot 1 . "$

Definição 4.10. Chama-se $\frac{X E}{2}$ o conjunto de elementos de $\Lambda E$ da forma $\eta \wedge$ Exp u, onde $u \in \Lambda E$ e onde $\eta \bar{e}$ um elemento decomponível de $\Lambda E$ (produtos exteriores de elementos de $\mathrm{E}$ e também os elementos de $\mathrm{K}$ ).

Proposição 4.11. [17] Se $x \in \operatorname{XE}$, então $F(x) \in \mathrm{XE}^{*}$ F é o isomorfismo definido na Proposição 4.6. 
Corolārio 4.12. [17] Se $x \in X E$ e $z^{\prime} \in X E^{*}$, então $x \tau z^{\prime} \in X E$.

Seja $B: E \times E \rightarrow K$ uma forma bilinear sobre $E$ t.q.

$$
B(a, a)=Q(a) \cdot \text { para todo } a \in E
$$

Pode-se associar a $\mathrm{B}$ um elemento de $\mathrm{E}^{*} \otimes \mathrm{E}^{*}$, que representare mos por $B$. Como $E^{*} \cdot E^{*}$ pode ser identificado com um subespaço de $\Lambda(E \oplus E)$, pode-se definir $\operatorname{Exp} B$ em $\Lambda(E \oplus E)^{*}$.

Definição 4.13. A álgebra $\Lambda(E, B)$ é o espaço vetorial $\Lambda E$ munido do produto que a todo par $(x, y)$ associa

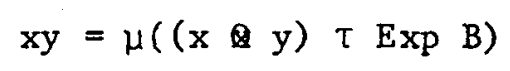

Para explicitar este produto, observemos que:

$$
\text { Se } \quad B=\sum_{i=1}^{p} x_{i}^{\prime} \otimes y_{i}^{\prime}=\sum_{i=1}^{p} u_{i}^{\prime}, \quad x_{i}^{\prime}, y_{i}^{\prime} \in E^{*}
$$

então

$$
\begin{aligned}
\operatorname{Exp} B & =\left(1+u_{1}^{\prime}\right) \wedge\left(1+u_{2}^{\prime}\right) \wedge \ldots \wedge\left(1+u_{p}^{\prime}\right) \\
& =\left[\left(1+u_{1}^{\prime}\right) \wedge\left(1+u_{2}^{\prime}\right)\right] \wedge \ldots \wedge\left(1+u_{p}^{\prime}\right) \\
& =\left[\left(1+u_{1}^{\prime}+u_{2}^{\prime}+u_{1}^{\prime} \wedge u_{2}^{\prime}\right)\right] \wedge \ldots \wedge\left(1+u_{p}^{\prime}\right)
\end{aligned}
$$

Se

$$
\begin{aligned}
\mathrm{p}=1, \mathrm{~B}=\mathrm{x}_{1}^{\prime} \otimes \mathrm{y}_{1}^{\prime} \Longrightarrow \operatorname{Exp} \mathrm{B} & =1+\mathrm{B} \\
& =1+\mathrm{x}_{1}^{\prime} \otimes \mathrm{y}_{1}^{\prime}
\end{aligned}
$$

(se $\mathrm{B}$ é um elemento decomponível de $\Lambda(\mathrm{E} \oplus \mathrm{E})^{*}$ ). 
Para

$$
\begin{aligned}
& \mathrm{p}=2, B=u_{1}^{\prime}+u_{2}^{\prime}=x_{1}^{\prime} y_{1}^{\prime}+x_{2}^{\prime} \otimes y_{2}^{\prime} \\
& \operatorname{Exp} B=1+u_{1}^{\prime}+u_{2}^{\prime}+u_{1}^{\prime} \wedge u_{2}^{\prime} \\
&=1+x_{1}^{\prime} \otimes y_{1}^{\prime}+x_{2}^{\prime} \otimes y_{2}^{\prime}+\left(x_{1}^{\prime} \otimes y_{1}^{\prime}\right) \wedge\left(x_{2}^{\prime} \otimes y_{2}^{\prime}\right) \\
& {\left[e \text { vale }\left(x_{1}^{\prime} \otimes y_{1}^{\prime}\right) \wedge \cdot\left(x_{2}^{\prime} \otimes y_{2}^{\prime}\right)=\sigma\left(y_{1}^{\prime}, x_{2}^{\prime}\right) \cdot\left(x_{1}^{\prime} \wedge x_{2}^{\prime}\right) \otimes\left(y_{1}^{\prime} \wedge y_{2}^{\prime}\right)\right.} \\
&\left.=1+x_{1}^{\prime} \otimes y_{1}^{\prime}+x_{2}^{\prime} \otimes y_{2}^{\prime}+\sigma\left(y_{1}^{\prime}, x_{2}^{\prime}\right) \cdot\left(x_{1}^{\prime} \wedge x_{2}^{\prime}\right) \otimes\left(y_{1}^{\prime} \wedge y_{2}^{\prime}\right)\right]
\end{aligned}
$$$$
\text { Neste caso, Exp B difere de } 1+\text { B por um elemento de grau } 4
$$$$
\operatorname{Exp} B \equiv 1+B \text { módulo } \stackrel{4}{\Lambda}(E \oplus E) \text { * }
$$

Para $p=j$

$$
\operatorname{Exp} B=1+B+C+D+\ldots+W
$$

onde

$$
\begin{aligned}
& \mathrm{c} \in \stackrel{2}{\Lambda \mathrm{L}}{ }^{*} \stackrel{2}{\Lambda \mathrm{LE}}{ }^{*} \subset \stackrel{4}{\Lambda}(\mathrm{E} \oplus \mathrm{E}){ }^{*} \quad(\mathrm{gr}(\mathrm{C})=4) \\
& D \in \stackrel{3}{\Lambda}^{*} \otimes \stackrel{3}{\Lambda E}^{*} \subset \stackrel{6}{\Lambda}(E \oplus E){ }^{*} \quad(\operatorname{gr}(D)=6)
\end{aligned}
$$

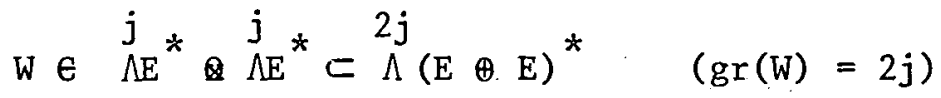

e de modo geral

$$
\operatorname{Exp} B \equiv 1+B \quad \text { módulo } \quad \stackrel{\geq 4}{\Lambda(E \oplus E)} \text { * }
$$

Verifica-se imediatamente que o escalar 1 é o elemento unidade para este produto (I); demonstraremos mais adiante sua associatividade. 
E óbvio que $\Lambda^{+} E$ e $\overline{\Lambda^{-}}$dão uma graduação de $\Lambda(E, B)$ sobre $\mathbb{Z} / 2 \mathbb{Z}$.

Lema 4.14. "Se $x$ e $\stackrel{p}{\Lambda E}$ e y $\in \stackrel{q}{\Lambda E}$, então

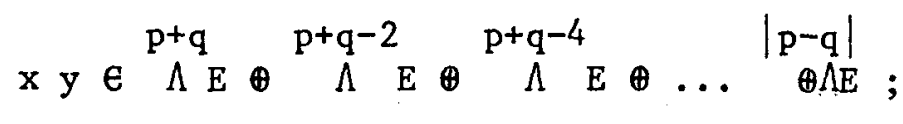

a componente de $x y$ em $\stackrel{p+q}{\Lambda} E \bar{e} x \wedge y . "$

\section{Prova}

Considerando-se a expressão de $\operatorname{Exp} B$, verifica-se que para todo grau par $2 j$, a componente de $\operatorname{Exp} B$ em $\Lambda^{2 j}(E \oplus E)^{*}$ está de fato em ${ }^{j} E^{*} \otimes{ }^{j} E^{*}$; e se $\mathrm{j}=0$, ela vale 1 .

\section{Então temos,}

se $\quad x \in \stackrel{p}{\Lambda}$ e $\quad$ y $\in \stackrel{q}{\Lambda}, \quad$ e $\quad B=\sum_{i=1}^{m} x_{i}^{\prime} \otimes y_{i}^{\prime} \quad x_{i}^{\prime}, y_{i}^{\prime} \in E^{*} \quad(1 \leq i \leq m)$

$$
\begin{aligned}
x \cdot y & =\mu((x \otimes y) \tau \operatorname{Exp} B)= \\
& =\mu((x \otimes y) \tau(1+B+C+\ldots+W))
\end{aligned}
$$

onde

$$
\begin{aligned}
& C=s \cdot\left(x_{1}^{\prime} \wedge x_{2}^{\prime}\right) \otimes\left(y_{1}^{\prime} \wedge y_{2}^{\prime}\right) \\
& D=s \quad\left(x_{1}^{\prime} \wedge x_{2}^{\prime} \wedge x_{3}^{\prime}\right) \otimes\left(y_{1}^{\prime} \wedge y_{2}^{\prime} \wedge y_{3}^{\prime}\right) \\
& W=s \cdot\left(x_{1}^{\prime} \wedge x_{2}^{\prime} \wedge \ldots \wedge x_{j}^{\prime}\right) \otimes\left(y_{1}^{\prime} \wedge y_{2}^{\prime} \wedge \ldots \wedge y_{j}^{\prime}\right)
\end{aligned}
$$

Alēm disso, observemos que

$$
\begin{aligned}
& (x \otimes y) \tau\left[\left(x_{1}^{\prime} \wedge \ldots \wedge x_{j}^{\prime}\right) \otimes\left(y_{1}^{\prime} \wedge \ldots \wedge y_{j}^{\prime}\right)\right]= \\
& {\left[x \tau\left(x_{1}^{\prime} \wedge \ldots \wedge x_{j}^{\prime}\right)\right] \otimes\left[y^{\prime}\left(y_{1}^{\prime} \wedge . . \wedge y_{j}^{\prime}\right)\right]=} \\
& \left\{\left[\left(x \tau x_{1}^{\prime}\right)\right] \tau \ldots \tau x_{j}^{\prime}\right\} \otimes\left\{\left[\left(y \tau y_{1}^{\prime}\right) \tau y_{2}^{\prime}\right] \tau \ldots \tau y^{\prime}{ }_{j}\right\}
\end{aligned}
$$

OBS. a letra.s nas igualdades indica o sinal que precede a expressão, proveniente da definição de produto tensorial graduado. 
Lembrando-se que cada $x_{i}^{\prime}$ (ou $\left.y_{i}^{\prime}\right)(1 \leq i \leq m)$ determina uma anti-derivação $\tau x_{i}^{\prime}$ (ou $\tau y_{i}^{\prime}$ ) de $\Lambda E$, que é de grau -1 , verifica-se que o elemento definido por (15)

é de grau $p+q-2 j, 1 \leq j \leq m$.

$$
\begin{aligned}
(x \otimes y) \tau\left(A_{j}^{\prime} \otimes B_{j}^{\prime}\right) \quad A_{j}^{\prime} & =x_{1}^{\prime} \wedge x_{2}^{\prime} \wedge \cdots \wedge x_{j}^{\prime} \\
B_{j}^{\prime} & =y_{j}^{\prime} \wedge y_{2}^{\prime} \wedge \ldots \wedge y_{j}^{\prime}
\end{aligned}
$$

Além disso,

$$
(x \otimes y) \tau\left(A_{j}^{\prime} \otimes B_{j}^{\prime}\right)=0 \text { se } p+q<2 j \text { e }
$$

então os termos de Exp B que serão considerados vão até o grau $p+q-2 j$, istoé,

$$
g r(W)=p+q-2 j \text { e } \quad j=p \text { ou } j=q
$$

Portanto:

$$
\operatorname{gr}(W)= \begin{cases}p+q-2 p=q-p & \text { se } j=p \\ p+q-2 q=p-q & \text { se } j=q\end{cases}
$$

$\mathrm{W} \in \stackrel{\circ}{\Lambda} E=\mathrm{K}$ se $\mathrm{p}+\mathrm{q}$ é par e $\mathrm{W} \in \stackrel{1}{\Lambda E}=\mathrm{E}$ se $\mathrm{p}+\mathrm{q}$ é ímpar

$$
(p+q=2 j \text { ou } p+q=2 j+1)
$$

Lema 4.15. "Se $x, y \in E$, então $x \cdot y=x \wedge y+B(x, y)$; em particular

$$
x^{2}=Q(x) . "
$$

Prova

De fato, vimos que

$$
\operatorname{Exp} B=1+B \text { módulo } \stackrel{\geq 4}{\Lambda}(E \oplus E)^{*}
$$


Então

$$
\begin{aligned}
x \cdot y & =\mu((x-y) \tau(I+B)) \\
& =\mu((x \otimes y) \tau 1+(x y) \tau B)) \\
& =x \wedge y+B(x, y) \quad x, y \in E
\end{aligned}
$$

Os resultados dos dois lemas anteriores coincidem com as afirma ções do Teorema 4.8 de Chévalley e além disso, pela propriedade da aplicação uni versal, conclui-se que: existe um homomorfismo de álgebras de $C(E, Q)$ em $\Lambda(E, B)$.

Proposição 4.16. [17] "Sejam $B_{12}, B_{13}, B_{23}$ as imagens de B pelos três isomorfismos

$$
\begin{aligned}
& E^{*} \otimes E^{*} \rightarrow E^{*} \otimes E^{*} \otimes E^{*} \\
& E^{*} \otimes E^{*} \otimes E^{*} \\
& E^{*} E^{*} \rightarrow K^{*} E^{*} \otimes E^{*} ;
\end{aligned}
$$

considera-se $B_{12}, B_{13}, B_{23}$ como elementos de $\Lambda(E \oplus E \oplus E) *$ Se $\mathrm{x}, \mathrm{y}$ e $z \in \Lambda \mathrm{E}$, os produtos (xy) z e $\mathrm{x}(\mathrm{yz})$ são os dois iguais a

$$
\mu_{123}\left((x \otimes y) \tau \operatorname{Exp}\left(B_{12}+B_{13}+B_{23}\right)\right)
$$

se $\mu_{123}$ representa o homomorfismo evidente de $\Lambda(E \oplus E \oplus E)$ em $\Lambda E . "$ Esta proposição demonstra a associatividade de $\Lambda(E, B)$.

Proposição 4.17. "Existe um e um só isomorfismo $J_{B}$ da älgebra $C_{E}$ (ou $C(E, Q)$ ) sobre a á1gebra $\Lambda(E, B), t . q . J_{B}(a)=a$ para todo a $\in E$. Este iso morfismo respeita as graduações sobre $\mathbf{Z} / 2 \mathbb{Z}$; para todo $\mathrm{p} \in \mathbb{Z}$, ele envia $\stackrel{\leq}{C_{E}}$ sobre $\stackrel{\leq p}{\Lambda} E=k \oplus E \oplus \stackrel{2}{2} \oplus \ldots \oplus \stackrel{p}{\Lambda E} . "$ 


\section{Prova}

De fato por causa de [4.15] existe um homomorfismo (único) $J_{B}$ de $C_{E}$ em $\Lambda E$ t.q. $J_{B}(a)=$ a para todo a $E E$.

Se $a_{1}, a_{2}, \ldots, a_{p} \in E$, deduz-se de (4.14) que $a_{1} a_{2} \cdots a_{p}=a_{1} \wedge a_{2} \wedge \cdots \wedge a_{p}$ módulo $\stackrel{\leq p-2}{\Lambda} E ;$

o que permite demonstrar a bijetividade de $J_{B}$ e o fato que $J_{B}$ preserva as filtra ções.

Como B não é determinada de maneira única por $Q$, comparemos duas escolhas de B.

Proposição 4.18. "Sejam $B_{1}$ e $B_{2}$ duas formas bilineares sobre $E$ tais que

$$
B_{1}(a, a)=B_{2}(a, a)=Q(a) \text { para todo } a \in E
$$

existe $v \in \stackrel{2}{\Lambda} \mathbb{E}^{*}$ ta1 que, quaisquer que sejam a e $b \in E$,

$$
B_{1}(a, b)-B_{2}(a, b)=\langle v, a \wedge b\rangle
$$

a aplicação $x \rightarrow x$ Exp $v$ é o único isomorfismo da àlgebra $\Lambda\left(E, B_{1}\right)$ sobre a álgebra $\Lambda\left(E, B_{2}\right)$ que deixa fixo os elementos de E".

\section{Prova}

A existência de $v \bar{e}$ evidente.

0 produto de $x \tau \operatorname{Exp} v$ e y $\tau \operatorname{Exp} \vee \operatorname{em} \Lambda\left(E, B_{2}\right) \bar{e}$

$$
\mu\left((x \otimes y) \tau \operatorname{Exp}\left(B_{2}+v @ 1+1 \otimes v\right)\right)
$$

Comparemos este $\operatorname{com}\left(\mu(x @ y) \tau \operatorname{Exp} B_{1}\right) \tau \operatorname{Exp} v$, que por (5) é

igual à

$$
\mu\left((x \otimes y) \tau \operatorname{Exp}\left(B_{1}+\delta(v)\right)\right.
$$


Se provarmos que $B_{2}+v 1+1 v=B_{1}+\delta(v)$, a proposição es tá demonstrada.

$$
\begin{gathered}
\text { Ora, sejam } b_{1}^{\prime}, b_{2}^{\prime}, \ldots c_{1}^{\prime}, c_{2}^{\prime}, \ldots e E^{*} \text { t.q. } \\
v=\sum_{j} b_{j}^{\prime} \wedge c_{j}^{\prime} ;
\end{gathered}
$$

encontra-se

$$
\begin{aligned}
& B_{2}-B_{1}=\sum_{j}\left(b_{j}^{\prime} \otimes c_{j}^{\prime}-c_{j}^{\prime} \otimes b_{j}^{\prime}\right), \\
& \delta(v)=v \otimes 1+1 \otimes v+\sum\left(b_{j}^{\prime} \otimes c_{j}^{\prime}-c_{j}^{\prime} \otimes b_{j}^{\prime}\right),
\end{aligned}
$$

a igualdade anunciada é verificada.

Vemos que toda propriedade de $\Lambda(E, B)$ é também uma propriedade de $C(E, Q)$, graças ao isomorfismo $J_{B}$ da proposição 4.17 , e a sua expressão em $C(E, Q)$ não depende da escolha de B.

Também, por causa de $[4.18]$ e $[4.12]$, a imagem de XE em $C(E, Q)$ não depende de $B$; esta imagem $X(E, Q)=J_{B}^{-1}(X E)$ é estável pelo produto e coloca-se em evidência um sub-conjunto notável de $C(E, Q)$; J.H. mostra que o grupo de Clifford é o conjunto dos elementos inversíveis de $X(E, Q)$.

Após ter colocado a possibilidade de escolhas diferentes de B, vamos indicar as mais interessantes. Se $K$ não é de característica 2, temos a es colha canônica de $\mathrm{B}_{\mathrm{O}} \mathrm{B}_{\mathrm{O}}=-\frac{1}{2} \mathrm{~B}$, o que dá o isomorfismo linear determinado por Chevalley [8], e que é usado também de preferência neste texto.

Se E é a soma direta de dois subespaços $U$ e $V$ totalmente singulares, a forma bilinear $B$ associada ao par $(U, V)$ é definida:

$$
B(a, b)=\phi(a, b) \quad \text { se } a \in V \text { e } b \in U
$$

e

$$
B(U, E)=B(E, V)=0
$$


(onde $\phi \bar{e}$ a aplicação bilinear canonicamente associada à Q); esta escolha de B implica que

$$
x y=x \wedge y \quad \text { se } \quad x \in \Lambda U \text { ou se } y \in \Lambda V
$$

- que torna os cálculos mais fáceis, o isomorfismo.j $J_{B}$ é então a inversa da aplicação linear especial ("normal ordering"), que é introduzida em [28].

OBS. - Observemos que o produto interioràdireita tambēm é definido em $C(E, Q)$ $\left(C(E, Q) \times \Lambda E^{*}+C(E, Q)\right)$ com propriedades anālogas ao pritidi. definido em $\Lambda \mathrm{E}$.

A seguinte proposição generaliza o lema [4.15]. Se B é bilinear em E, (não necessariamente simétrica), sejamB' e B" as aplicações lineares de $E$ em $E^{*}$ definidas

$$
B^{\prime}(a)(b)=B^{\prime \prime}(b)(a)=B(a, b) \quad a, b \in E
$$

Representa-se $\phi$ a forma bilinear canonicamente associada à $Q$ (simétrica) e $\phi$ a sua aplicação linear associada

$$
\left(\phi: E \rightarrow E^{*}\right)
$$

Proposição 4.19. Se a $\in \mathrm{E}$ e $\mathrm{x} \in \Lambda \mathrm{E}$, temos em $\Lambda(\mathrm{E}, \mathrm{B})$ :

$$
\begin{aligned}
& \text { (a) } \text { ax }=a \wedge x+x \tau B^{\prime}(a) \\
& \text { (b) } \tilde{x a}=a \wedge x-x \tau B^{\prime \prime}(a) \\
& \text { (c) } \mathrm{ax}-\tilde{\mathrm{xa}}=\mathrm{x} \tau \phi \text { (a) } \\
& \text { (d) } a x+\tilde{x a}=2 a \wedge x \text { se } B=\phi / 2
\end{aligned}
$$

\section{Prova}

As identidades (a) e (b) resultam dos cálculos seguintes:

$$
\begin{aligned}
& (a @ x) \tau B=1 \otimes\left(x \tau B^{\prime}(a)\right) \\
& (x \otimes a) \tau B=-\left(\tilde{x} \tau B^{\prime \prime}(a) \otimes I\right.
\end{aligned}
$$


Pode-se deduzir (b) de (a) e de (25) [17].

Como $\phi(a, b)=B(a, b)+B(b, a)$, deduz-se (c) de (a) e (b); graças ao produto interior à direita em $C(E, Q)$, a identidade (c) é também válida em $C(E, Q) . \Gamma$

Se $B=\phi / 2$, então $B^{\prime}=B^{\prime \prime}$, o que implica (d); esta identidade mostra que todo automorfismo (respectivamente, toda derivação) da älgebra $\Lambda(E, \phi / 2)$ que deixa $\mathrm{E}$ invariante, ē também um automorfismo (respectivamente uma derivação) da älgebra $\Lambda$ E.

Vejamos como a ālgebra $C_{M}$ e $C_{E}$ podem ser construídas e analisadas, usando este procedimento.

Seja $\sigma: E \rightarrow E^{*}$ o isomorfismo que se obtēm, restringindo a apliça ção ortogonal $F \in G$, ao subespaço $E$ de $M$. Temos a forma bilinear $B_{1}(x, y)=\sigma(x)(y)$ $(x, y \in E)$ e o espaço quadrático $\Lambda\left(E, B_{1}\right)$.

A àlgebra $\Lambda\left(E, B_{1}\right)$ é então definida pelo produto

$$
x y=x \wedge y+\delta_{x^{\prime}}(y) \text { onde } x \in E, y \in \Lambda E
$$

$\delta_{x^{\prime}}$ é a anti-derivação de $\Lambda E$ t.q.

$$
\delta_{x^{\prime}}(y)=x^{\prime}(y)=B_{1}(x, y) \text { se } y \in E
$$

Sabemos que a toda forma bilinear $\mathrm{B}: \mathrm{E} \times \mathrm{E} \rightarrow \mathrm{K}$ pode se associar um elemento de $E^{*} \otimes E^{*}$, através do isomorfismo

$$
\begin{gathered}
B(E) \cong E^{*} \otimes E^{*} \\
B \leftrightarrow x^{\prime} \otimes y^{\prime} \text { t.q. } \quad\left(x^{\prime} \otimes y^{\prime}\right)(a, b)=x^{\prime}(a) \cdot y^{\prime}(b) \quad a, b \in E
\end{gathered}
$$

é chamado o produto das formas lineares $x^{\prime}$ e $y^{\prime} \in E^{*}$

Se $B: E \times E \rightarrow K$ é uma forma bilinear $\left(B \leftrightarrow x^{\prime} \otimes y^{\prime}\right)$ e $B_{1} \bar{e} \operatorname{defi-}$ nida como anteriormente, temos

$$
B(a, b)=\left(x^{\prime} @ y^{\prime}\right)(a, b)=x^{\prime}(a) \cdot y^{\prime}(b)=B_{1}(x, a) \cdot B_{1}(y, b)
$$




\section{Temos então}

$$
\delta_{x^{\prime}}: \Lambda E \rightarrow \Lambda E \text { e } \delta_{y^{\prime}}: \Lambda E \rightarrow \Lambda E
$$

anti-derivações $\mathrm{e}$

$$
\delta_{x^{\prime}} \otimes \delta_{y^{\prime}}: \Lambda E \widehat{Q} \Lambda E \rightarrow \Lambda E \widehat{Q} \Lambda E
$$

é uma anti-derivação de $\Lambda(E \oplus E) t \cdot q \cdot \delta_{x^{\prime}} \otimes \delta_{y^{\prime}}(a \otimes b)=B(a, b)$ se $a, b \in E$. Dessa forma, temos a àlgebra $\Lambda(E, B)$, cujo produto $\bar{e}$ dado por (11); no desenvolvimento deste produto, utiliza-se a anti-derivação que é a gene ralização da anti-derivação $\delta_{x}$ introduzida por Chevalley [8] para definir o produto de cilifford.

Usando as propriedades do produto interior e o desenvolvimento de $\operatorname{Exp} B$, verifica-se que o produto definido em $[11]$ coincide com o produto de Clifford, dado por Chevalley [8], se B é a forma bilinear canonicamente associada a Q. 


\section{BIBLIOGRAFIA}

[1] ARTIN, E. - Geometric Algebra. New York, Interscience, 1957.

[2] ATIYAH, M.F.; BOTT, R. \& SHAPIRO, A. - Clifford Modules. Topology (3): 3 $-39,1964$.

[3] BAER, R. - Linear Algebra and Projective Geometry. New York, Academic Press, 1952.

[4] BOURBAKI, N. - Algèbre Lineäire. Algèbre, Paris, Hermann, 1948. Cap. II (Eléments de Mathématique).

[5] BOURBAKI, N. - Algèbre Multilineaire. Algèbre, Paris, Hermann, 1948. Cap. III (Eléments de Mathématique).

[6] BOURBAKI, N. - Formes Sesquilinēaires et Formes Quadratiques. Algèbre, Paris, Hermann, 1959. Cap. IX (Eléments de Mathëmatique).

[7] CHAMBADAL, L. \& OVAER, J.L. - Algèbre Lineaire et Algèbre Tensorialle. Paris, Dunod, 1968.

[8] CHEVALLEY, C. - The Algebraic Theory of Spinors. New York, Columbia University Press, 1954 .

[9] CHEVALlEY, C. - Théorie des Groupes de Lie. Paris, Hermann, 1954, 3v.

[10] CHEVALLEY, C. - The Construction and Study of Certain Important Algebras. Tokyo, The Math. Soc. of Japan, 1955. 
[11] CHEVALLEY, C. - Fundamental Concepts of Algebra. New York, Academic Press, 1956.

[12] CRUMEYROLLE, A. - Algèbre de Clifford Symplectique. J. Math. Pure Appl., 56: $205-30,1977$.

[13] DEHEUVELS, R. - Formes Quadratiques et Groupes Classiques. Paris, Presses * Universitaires de France, 1981.

[14] DIEUDONNE, ..J. - La Géométrie des Groupes Classiques. Berlim, Springers-Verlag, 1955.

[15] DIEUDONNE, J. - Sur les Groupes Classiques. Paris, Hermann, 1948.

[16] GRUNENFELDER, L. - Clifford K-Algebras and $\mathrm{K}^{*}$-Groups. Math. Z., 185: 137 $-50,1984$.

[17] HELMSTETTER, J. - Groupe de Clifford Pour des Formes Quadratiques de Rang Quelconque. C.R. Acad. Sc. Paris, 285: $175-77,1977$.

[18] HELMSTETtER, J. - Prodúts Intérieurs de Séries Formelles et Algèbres de Clifford Symplectiques. C.R. Acad. Sc. Paris, 293: $63-65,1981$.

[19] JACOBSON, N. - Lectures in Abstract Algebra. New Jersey, D. Van Nostrand, 1951.

[20] LAM, T.Y. - The Algebraic Theory of Quadratic Forms. Massachusetts, Benja$\min , 1973$.

[21] LIMA, E.L. - Cálculo Tensorial. Rio de Janeiro, IMPA, 1965. 
[22] LOUNESTO, P. - Sur les Idéaux à Gauche des Algèbres de Clifford et les Produits Scalaires des Spineurs. Ann. Inst. Henri Poincaré, 33 (1): 53$-61,1980$.

[23] MICALI, A. \& REVOY, P. - Modules Quadratiques. Bull. Soc. Math. France Mémoire, 63, 1979.

[24] MICALI, A. \& VILLAMAYOR, O.E. - Sur Les Algèbres de Clifford. Ann. Scient. Ec. Norm. Sup., $1271-304,1968$.

[25] MILNOR, J. \& HUSEMOLLER, D. - Symetric Bilinear Forms. New York, Springer-Verlag, 1973.

[26] O'MEARA, 0.T. - Introduction to Quadratic Forms. New York, Springer-Verlag, 1973.

[27] REVOY, P. \& GARNIER, M.R. - Nouvelles Algèbres de Clifford. C.R. Acad. Sc. Paris, 284: $985-87,1977$.

[28] SATO, M.; MIWA, T. \& JIMBO, M. - Holonomic Quantum Fields I.

Publ. RIMS Kyoto Univ., 14: $223-67,1968$.

[29] SCHEMBERG, M. - On the Grassmann and Clifford Algebras I. An. Acad. Bras. Ciênc., 28: 11 - 19, Marc., 1956

[30] SCHEMBERG, M. - On the Grassmann and Clifford Algebras II. An. Acad. Bras. Ciênc., 32 (3/4): $299-322,1960$.

[31] ZaSSenhaus, H. - On the Spinor Norm. Arch. Math., 13: $434-51,1962$. 